\title{
Synthesis of 4,7-Difunctionalized Indoles via Imino Exchange and Sulfinyl Migration
}

\author{
Xiaohua Li, Lei Li, Weiyi Wang, Qiuqin He* and Renhua Fan*. \\ Department of Chemistry, Fudan University, 2005 Songhu Road, Shanghai 200438, \\ China.
}

\section{Supporting Information}

1. General experimental methods (S2)

2. Representative procedure and characterization data. (S2-S18)

3. X-ray diffraction structure of compound 2 (S19)

4. Copies of ${ }^{1} \mathrm{H},{ }^{13} \mathrm{C}$ NMR spectra of products (S20-S102) 


\section{General experimental methods}

All reactions were performed in Schlenk tubes under nitrogen atmosphere. Flash column chromatography was performed using silica gel (60-Å pore size, $32-63 \mu \mathrm{m}$, standard grade). Analytical thin-layer chromatography was performed using glass plates pre-coated with $0.25 \mathrm{~mm} 230-400$ mesh silica gel impregnated with a fluorescent indicator $(254 \mathrm{~nm})$. Thin layer chromatography plates were visualized by exposure to ultraviolet light. Organic solutions were concentrated on rotary evaporators at $\sim 20$ Torr (house vacuum) at $35-40{ }^{\circ} \mathrm{C}$. Commercial reagents and solvents were used as received. Nuclear magnetic resonance (NMR) spectra are recorded in parts per million from internal tetramethylsilane on the $\delta$ scale. For reactions that require heating, oil bath is used as the heat source.

\section{Representative procedure and characterization data}

\subsection{Synthesis of compounds 1}

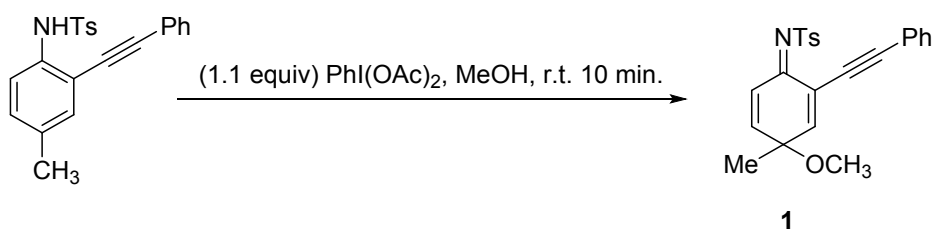

$\mathrm{PhI}(\mathrm{OAc})_{2}(350 \mathrm{mg}, 1.1 \mathrm{mmol})$ was added into the solution of 2-alkynyl aniline (360 $\mathrm{mg}, 1.0 \mathrm{mmol})$ in $\mathrm{MeOH}(20 \mathrm{~mL})$ at $25^{\circ} \mathrm{C}$. After $10 \mathrm{~min}$, the reaction mixture was quenched with saturated $\mathrm{NaHCO}_{3}(20 \mathrm{~mL})$, and extracted by ethyl acetate $(50 \mathrm{~mL}$ x 3$)$. The organic layer was dried over anhydrated $\mathrm{Na}_{2} \mathrm{SO}_{4}$, and concentrated in vacuo. The residue waspurified by flash column chromatography on silica gel (petroleum ether /ethyl acetate $=8: 1)$ to afford 2-alkynycyclohexadienimine 1 (371 mg, 95\% yield).

\subsection{Representative procedure}

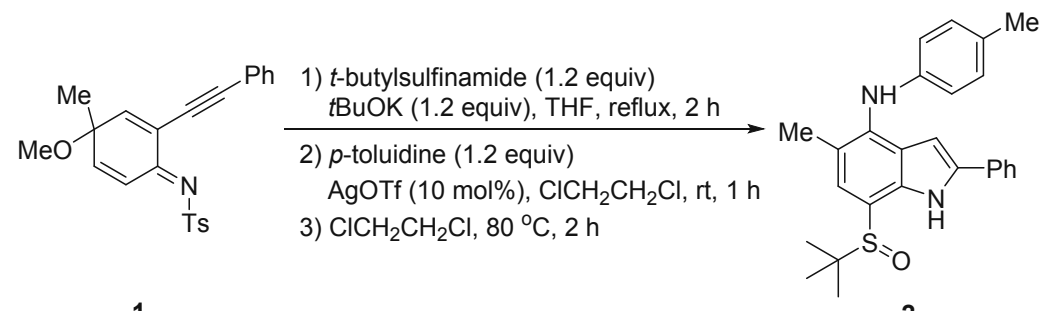


tert-Butanesulfinamide $(0.12 \mathrm{mmol})$ and potassium t-butoxide $(0.12 \mathrm{mmol})$ were added to a solution of 2-alkynylcyclohexadienimine $1(0.1 \mathrm{mmol})$ in THF $(2 \mathrm{~mL})$. The reaction mixture was stirred at the refluxing temperature for $2 \mathrm{~h}$. The crude product was purified by a short column chromatography on silica gel (eluent: petroleum ether/EtOAc 4:1). The isolated imino exchange intermediate was dissolved in dichloroethane $(2 \mathrm{~mL})$ and treated with $p$-toluidine $(0.12 \mathrm{mmol})$ and $\operatorname{AgOTf}(0.01$ mmol). The reaction mixture was stirred at room temperature for $1 \mathrm{~h}$, then was filtered through a short silica gel column to remove AgOTf. The resulting filtrate was stirred at $80^{\circ} \mathrm{C}$. After the intermediate was completely consumed (monitored by TLC analysis), the mixture was purified by flash column chromatography on silica gel (eluent: petroleum ether/EtOAc 5:1) to furnish compound 2, $30 \mathrm{mg}, 72 \%$ yield.

\subsection{Representative procedure in $1 \mathrm{mmol}$ scale}

tert-Butanesulfinamide $(1.2 \mathrm{mmol})$ and potassium $t$-butoxide $(1.2 \mathrm{mmol})$ were added to a solution of 2-alkynylcyclohexadienimine $1(1 \mathrm{mmol})$ in THF $(10 \mathrm{~mL})$. The reaction mixture was stirred at the refluxing temperature for $2 \mathrm{~h}$. The crude product was purified by a short column chromatography on silica gel (eluent: petroleum ether/EtOAc 4:1). The isolated imino exchange intermediate was dissolved in dichloroethane $(10 \mathrm{~mL})$ and treated with $p$-toluidine $(1.2 \mathrm{mmol})$ and $\operatorname{AgOTf}(0.1 \mathrm{mmol})$. The reaction mixture was stirred at room temperature for $1 \mathrm{~h}$, then was filtered through a short silica gel column to remove AgOTf. The resulting filtrate was stirred at $80^{\circ} \mathrm{C}$. After the intermediate was completely consumed (monitored by TLC analysis), the mixture was purified by flash column chromatography on silica gel (eluent: petroleum ether/EtOAc 5:1) to furnish compound 2, $287 \mathrm{mg}, 69 \%$ yield.

\subsection{Characterization data}

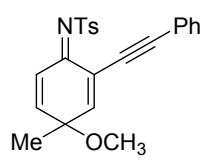

(Z)-N-(4-methoxy-4-methyl-2-(phenylethynyl)cyclohexa-2,5-dien-1-ylidene)-4-

methylbenzenesulfonamide 1: (eluent: petroleum ether/EtOAc 8:1), $371 \mathrm{mg}, 95 \%$; colorless solid; m.p. $155-156{ }^{\circ} \mathrm{C} ;{ }^{1} \mathrm{H}$ NMR $\left(400 \mathrm{MHz}, \mathrm{CD}_{3} \mathrm{CN}\right) \delta 7.94(\mathrm{~d}, J=8.3 \mathrm{~Hz}$, 2H), $7.57(\mathrm{~d}, J=10.4 \mathrm{~Hz}, 1 \mathrm{H}), 7.44(\mathrm{~m}, 7 \mathrm{H}), 7.16(\mathrm{~d}, J=2.6 \mathrm{~Hz}, 1 \mathrm{H}), 6.93(\mathrm{dd}, J=$ 
$10.4 \mathrm{~Hz}, 2.6 \mathrm{~Hz}, 1 \mathrm{H}), 3.12$ (s, 3H), 2.44 (s, 3H), 1.41 (s, 3H). (ref: Wang, L. F.; Fan, R. H. Org. Lett. 2012, 14, 3596-3599.)

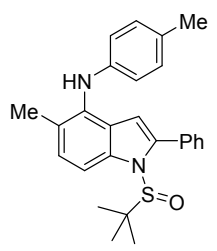

1-(tert-butylsulfinyl)-5-methyl-2-phenyl- $N$-(p-tolyl)-1H-indol-4-amine int-2B: (eluent: petroleum ether/EtOAc 8:1), $38 \mathrm{mg}, 92 \%$; gray solid; m.p. $110-111{ }^{\circ} \mathrm{C} ;{ }^{1} \mathrm{H}$ NMR (400 MHz, $\left.\mathrm{CDCl}_{3}\right) \delta 8.05(\mathrm{~d}, J=8.4 \mathrm{~Hz}, 1 \mathrm{H}), 7.51(\mathrm{~d}, J=7.0 \mathrm{~Hz}, 2 \mathrm{H}), 7.40(\mathrm{~m}$, $3 \mathrm{H}), 7.11(\mathrm{~d}, J=8.5 \mathrm{~Hz}, 1 \mathrm{H}), 7.00(\mathrm{~d}, J=8.2 \mathrm{~Hz}, 2 \mathrm{H}), 6.63(\mathrm{~d}, J=8.4 \mathrm{~Hz}, 2 \mathrm{H}), 6.38$ $(\mathrm{s}, 1 \mathrm{H}), 5.51(\mathrm{~s}, 1 \mathrm{H}), 2.32(\mathrm{~s}, 3 \mathrm{H}), 2.26(\mathrm{~s}, 3 \mathrm{H}), 1.07(\mathrm{~s}, 9 \mathrm{H}) .{ }^{13} \mathrm{C}$ NMR (100 MHz, $\left.\mathrm{CDCl}_{3}\right) \delta 144.3,141.2,139.0,132.0,131.7,130.0,129.5,128.2,128.1,126.6,126.1$, 125.8, 115.0, 113.0, 105.2, 64.4, 24.0, 20.4, 17.5. HRMS m/z calcd for $\mathrm{C}_{26} \mathrm{H}_{29} \mathrm{~N}_{2} \mathrm{OS}$ $\left([\mathrm{M}+\mathrm{H}]^{+}\right):$417.1995, found 417.1995.

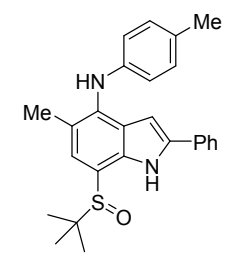

7-(tert-butylsulfinyl)-5-methyl-2-phenyl- $N$-(p-tolyl)-1H-indol-4-amine 2: (eluent: petroleum ether/EtOAc 5:1), $30 \mathrm{mg}, 72 \%$; gray solid; m.p. $105-106{ }^{\circ} \mathrm{C} ;{ }^{1} \mathrm{H}$ NMR (400 $\left.\mathrm{MHz} \mathrm{CDCl}_{3}\right) \delta 10.50(\mathrm{~s}, 1 \mathrm{H}), 7.57(\mathrm{~d}, J=7.7 \mathrm{~Hz}, 2 \mathrm{H}), 7.37$ (t, $\left.J=7.7 \mathrm{~Hz}, 2 \mathrm{H}\right), 7.27$ (t, $J=7.2 \mathrm{~Hz}, 1 \mathrm{H}), 7.04(\mathrm{~d}, J=7.9 \mathrm{~Hz}, 2 \mathrm{H}), 6.88(\mathrm{~s}, 1 \mathrm{H}), 6.77(\mathrm{~d}, J=8.2 \mathrm{~Hz}, 2 \mathrm{H}), 6.38$ $(\mathrm{d}, J=2.4 \mathrm{~Hz}, 1 \mathrm{H}), 2.30(\mathrm{~s}, 6 \mathrm{H}), 1.31(\mathrm{~s}, 9 \mathrm{H}) .{ }^{13} \mathrm{C} \mathrm{NMR}\left(100 \mathrm{MHz}, \mathrm{CDCl}_{3}\right) \delta 141.7$, $137.9,136.5,135.7,131.8,129.8,129.5,128.8,127.8,125.3,125.1,124.3,119.1$, 117.4, 112.8, 97.4, 58.2, 23.2, 20.6, 17.8. HRMS m/z calcd for $\mathrm{C}_{26} \mathrm{H}_{29} \mathrm{~N}_{2} \mathrm{OS}\left([\mathrm{M}+\mathrm{H}]^{+}\right)$: 417.1995, found 417.1976.

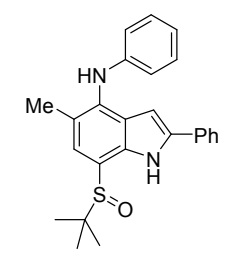

7-(tert-butylsulfinyl)-5-methyl- $N$,2-diphenyl-1H-indol-4-amine $\quad 3: \quad$ (eluent: petroleum ether/EtOAc 5:1), $29 \mathrm{mg}, 72 \%$; brown solid; m.p. 159-160 ${ }^{\circ} \mathrm{C} ;{ }^{1} \mathrm{H}$ NMR (400 $\left.\mathrm{MHz}, \mathrm{CDCl}_{3}\right) \delta 10.51(\mathrm{~s}, 1 \mathrm{H}), 7.57(\mathrm{~d}, J=7.4 \mathrm{~Hz}, 2 \mathrm{H}), 7.36(\mathrm{t}, J=7.6 \mathrm{~Hz}, 2 \mathrm{H}), 7.30$ $(\mathrm{d}, J=7.3 \mathrm{~Hz}, 1 \mathrm{H})$, , 7.24-7.21 (m, 3H), $6.89(\mathrm{~s}, 1 \mathrm{H}), 6.83(\mathrm{~d}, J=7.7 \mathrm{~Hz}, 2 \mathrm{H}), 6.42(\mathrm{~d}$, 
$J=2.3 \mathrm{~Hz}, 1 \mathrm{H}), 5.79(\mathrm{~s}, 1 \mathrm{H}), 2.31(\mathrm{~s}, 3 \mathrm{H}), 1.31(\mathrm{~s}, 9 \mathrm{H}) ;{ }^{13} \mathrm{C} \mathrm{NMR}\left(100 \mathrm{MHz}, \mathrm{CDCl}_{3}\right)$ $\delta 144.3,138.1,136.4,135.0,131.7,129.0,128.9,127.9,125.7,125.3,124.2,120.1$, 120.1, 116.7, 113.6, 97.2, 58.2, 23.2, 17.8. HRMS m/z calcd for $\mathrm{C}_{25} \mathrm{H}_{27} \mathrm{~N}_{2} \mathrm{OS}$ $\left([\mathrm{M}+\mathrm{H}]^{+}\right)$: 403.1839, found 403.1831.

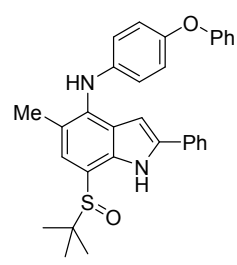

7-(tert-butylsulfinyl)-5-methyl- $N$-(4-phenoxyphenyl)-2-phenyl-1 $H$-indol-4-amine

4: (eluent: petroleum ether/EtOAc 5:1), $32 \mathrm{mg}, 65 \%$; gray solid; m.p. $112-113{ }^{\circ} \mathrm{C} ;{ }^{1} \mathrm{H}$ NMR (400 MHz, $\left.\mathrm{CDCl}_{3}\right) \delta 10.51(\mathrm{~s}, 1 \mathrm{H}), 7.58(\mathrm{~d}, J=7.6 \mathrm{~Hz}, 2 \mathrm{H}), 7.40(\mathrm{t}, J=7.6 \mathrm{~Hz}$, 2H), 7.32-7.25 (m, 3H), 7.05-6.94 (m, 6H), 6.88-6.86 (m, 3H), 6.35(s, 1H), 2.33(s, 3H), 1.33(s, 9H); ${ }^{13} \mathrm{C} \mathrm{NMR}\left(100 \mathrm{MHz}, \mathrm{CDCl}_{3}\right) \delta 150.4,140.2,137.9,136.5,135.78,129.6$, $128.9,127.8,125.3,124.7,124.4,122.4,120.4,119.07,118.8,117.6,112.7,97.3,58.2$, 23.2, 17.8. HRMS m/z calcd for $\mathrm{C}_{31} \mathrm{H}_{31} \mathrm{~N}_{2} \mathrm{O}_{2} \mathrm{~S}\left([\mathrm{M}+\mathrm{H}]^{+}\right)$: 495.2101, found 495.2078.

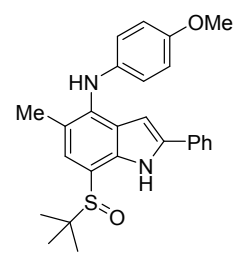

7-(tert-butylsulfinyl)- $\mathrm{N}$-(4-methoxyphenyl)-5-methyl-2-phenyl- $1 \mathrm{H}$-indol-4-amine 5: (eluent: petroleum ether/EtOAc 5:1), $29 \mathrm{mg}, 68 \%$; gray solid; m.p. 102-103 ${ }^{\circ} \mathrm{C} ;{ }^{1} \mathrm{H}$ NMR (400 MHz, $\left.\mathrm{CDCl}_{3}\right) \delta 10.48(\mathrm{~s}, 1 \mathrm{H}), 7.54(\mathrm{~d}, J=7.4 \mathrm{~Hz}, 2 \mathrm{H}), 7.36(\mathrm{t}, J=7.6 \mathrm{~Hz}$, $3 \mathrm{H}), 7.27(\mathrm{t}, J=7.7 \mathrm{~Hz}, 1 \mathrm{H}), 6.90(\mathrm{~d}, J=8.9 \mathrm{~Hz}, 2 \mathrm{H}), 6.87(\mathrm{~s}, 1 \mathrm{H}), 6.83(\mathrm{~d}, J=8.9 \mathrm{~Hz}$, $2 \mathrm{H}), 6.23(\mathrm{~d}, J=2.4 \mathrm{~Hz}, 1 \mathrm{H}), 5.66(\mathrm{~s}, 1 \mathrm{H}), 3.80(\mathrm{~s}, 3 \mathrm{H}), 2.30(\mathrm{~s}, 3 \mathrm{H}), 1.30(\mathrm{~s}, 9 \mathrm{H}) ;{ }^{13} \mathrm{C}$ NMR (100 MHz, $\left.\mathrm{CDCl}_{3}\right)$ 154.7, 137.5, 137.2, 136.9, 136.7, 131.8, 128.8, 127.7, 125.2, $124.54,123.5,120.5,117.1,114.3,111.3,97.5,58.1,55.6,23.2,17.78$. HRMS m/z calcd for $\mathrm{C}_{26} \mathrm{H}_{29} \mathrm{~N}_{2} \mathrm{O}_{2} \mathrm{~S}\left([\mathrm{M}+\mathrm{H}]^{+}\right)$: 433.1944, found 433.1944 .

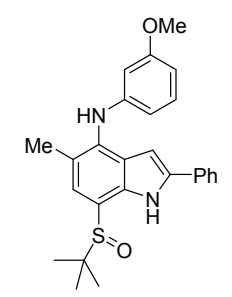

7-(tert-butylsulfinyl)- $\mathrm{N}$-(3-methoxyphenyl)-5-methyl-2-phenyl-1 $H$-indol-4-amine 6: (eluent: petroleum ether/EtOAc 5:1), $26 \mathrm{mg}, 60 \%$; gray solid; m.p. $164-165{ }^{\circ} \mathrm{C} ;{ }^{1} \mathrm{H}$ 
NMR (400 MHz, $\left.\mathrm{CDCl}_{3}\right) \delta 10.52(\mathrm{~s}, 1 \mathrm{H}), 7.60(\mathrm{~d}, J=7.1 \mathrm{~Hz}, 2 \mathrm{H}), 7.38(\mathrm{t}, J=7.6 \mathrm{~Hz}$, 2H), $7.28(\mathrm{t}, J=7.1 \mathrm{~Hz}, 1 \mathrm{H}), 7.13(\mathrm{t}, J=8.1 \mathrm{~Hz}, 1 \mathrm{H}), 6.90(\mathrm{~s}, 1 \mathrm{H}), 6.49$ (d, $J=2.5 \mathrm{~Hz}$, $1 \mathrm{H}), 6.44(\mathrm{td}, J=8.1,2.3 \mathrm{~Hz}, 2 \mathrm{H}), 6.36(\mathrm{t}, J=2.3 \mathrm{~Hz}, 1 \mathrm{H}), 5.75(\mathrm{~s}, 1 \mathrm{H}), 3.73(\mathrm{~s}, 3 \mathrm{H})$, 2.32 (s, 3H), 1.31 (s, 9H); ${ }^{13} \mathrm{C}$ NMR (100 MHz, $\left.\mathrm{CDCl}_{3}\right) \delta 160.6,145.9,138.3,136.3$, 134.7, 131.8, 129.8, 128.9, 127.9, 126.3, 125.3, 124.1, 120.8, 114.2, 109.2, 105.3, 102.2, 97.2, 58.2, 55.1, 23.2, 17.8. HRMS m/z calcd for $\mathrm{C}_{26} \mathrm{H}_{29} \mathrm{~N}_{2} \mathrm{O}_{2} \mathrm{~S}\left([\mathrm{M}+\mathrm{H}]^{+}\right)$: 433.1944, found 433.1933 .

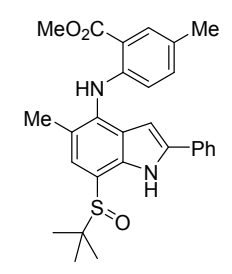

\section{Methyl-2-((7-(tert-butylsulfinyl)-5-methyl-2-phenyl-1H-indol-4-yl)amino)-5-}

methylbenzoate 7: (eluent: petroleum ether/EtOAc 5:1), $15 \mathrm{mg}, 32 \%$; gray solid; m.p. 117-118 ${ }^{\circ} \mathrm{C} ;{ }^{1} \mathrm{H}$ NMR $\left(400 \mathrm{MHz}, \mathrm{CDCl}_{3}\right) \delta 10.53$ (s, 1H), 7.59 (dt, $J=7.7 \mathrm{~Hz}, 1.7 \mathrm{~Hz}$, 2H), 7.43 (t, $J=2.5 \mathrm{~Hz}, 1 \mathrm{H}), 7.38$ (td, $J=7.7,2.3 \mathrm{~Hz}, 2 \mathrm{H}), 7.30$ (d, $J=7.4 \mathrm{~Hz}, 1 \mathrm{H})$, $7.08(\mathrm{dd}, J=8.3 \mathrm{~Hz}, 2.3 \mathrm{~Hz}, 1 \mathrm{H}), 6.90(\mathrm{~s}, 1 \mathrm{H}), 6.81(\mathrm{dt}, J=8.3,2.5 \mathrm{~Hz}, 1 \mathrm{H}), 6.39$ (d, $J=2.5 \mathrm{~Hz}, 1 \mathrm{H}), 5.76(\mathrm{~s}, 1 \mathrm{H}), 3.85(\mathrm{~s}, 3 \mathrm{H}), 2.52(\mathrm{~s}, 3 \mathrm{H}), 2.31(\mathrm{~s}, 3 \mathrm{H}), 1.32(\mathrm{~s}, 9 \mathrm{H}) ;{ }^{13} \mathrm{C}$ $\mathrm{NMR}\left(100 \mathrm{MHz}, \mathrm{CDCl}_{3}\right) \delta 142.1,138.3,136.4,134.7,132.2,131.7,131.3,130.0,128.9$, 127.9, 125.6, 125.3, 124.2, 120.1, 120.0, 118.7, 113.8, 97.1, 58.2, 51.8, 23.2, 20.8, 17.8. HRMS m/z calcd for $\mathrm{C}_{28} \mathrm{H}_{31} \mathrm{~N}_{2} \mathrm{O}_{3} \mathrm{~S}\left([\mathrm{M}+\mathrm{H}]^{+}\right)$: 475.2050, found 475.2030.

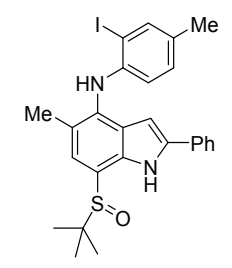

7-(tert-butylsulfinyl)- $\mathrm{N}$-(2-iodo-4-methylphenyl)-5-methyl-2-phenyl-1 $\mathrm{H}$-indol-4-

amine 8: (eluent: petroleum ether/EtOAc 5:1), $28 \mathrm{mg}$, 52\%; gray solid; m.p. 177$178{ }^{\circ} \mathrm{C} ;{ }^{1} \mathrm{H}$ NMR $\left(400 \mathrm{MHz}, \mathrm{CDCl}_{3}\right) \delta 10.54(\mathrm{~s}, 1 \mathrm{H}), 7.64(\mathrm{t}, J=7.8 \mathrm{~Hz}, 3 \mathrm{H}), 7.42(\mathrm{t}$, $J=7.6 \mathrm{~Hz}, 2 \mathrm{H}), 7.32$ (t, $J=7.4 \mathrm{~Hz}, 1 \mathrm{H}), 6.94(\mathrm{~s}, 1 \mathrm{H}), 6.44$ (d, $J=8.2 \mathrm{~Hz}, 1 \mathrm{H}), 6.31$ (s, 1H), $5.92(\mathrm{~s}, 1 \mathrm{H}), 2.31(\mathrm{~s}, 3 \mathrm{H}), 2.11(\mathrm{~s}, 3 \mathrm{H}), 1.33(\mathrm{~s}, 9 \mathrm{H}) ;{ }^{13} \mathrm{C} \mathrm{NMR}\left(100 \mathrm{MHz}, \mathrm{CDCl}_{3}\right)$ $\delta 144.2,139.2,138.8,138.5,136.1,134.2,131.6,128.9,128.0,127.3,125.4,124.0$, $122.5,122.0,115.5,112.7,96.9,82.8,58.2,23.2,21.3,17.8$. HRMS m/z calcd for $\mathrm{C}_{26} \mathrm{H}_{28} \mathrm{IN}{ }_{2} \mathrm{OS}\left([\mathrm{M}+\mathrm{H}]^{+}\right)$: 543.0962, found 543.0956. 


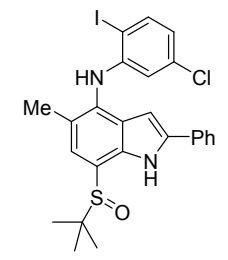

7-(tert-butylsulfinyl)- $\mathrm{N}$-(5-chloro-2-iodophenyl)-5-methyl-2-phenyl-1H-indol-4-

amine 9: (eluent: petroleum ether/EtOAc 5:1), $34 \mathrm{mg}, 60 \%$; gray solid; m.p. 176$177{ }^{\circ} \mathrm{C} ;{ }^{1} \mathrm{H}$ NMR $\left(400 \mathrm{MHz}, \mathrm{CDCl}_{3}\right) \delta 10.59(\mathrm{~s}, 1 \mathrm{H}), 7.62(\mathrm{~d}, J=7.5 \mathrm{~Hz}, 2 \mathrm{H}), 7.40(\mathrm{t}$, $J=7.6 \mathrm{~Hz}, 2 \mathrm{H}), 7.31(\mathrm{t}, J=7.3 \mathrm{~Hz}, 1 \mathrm{H}), 7.00(\mathrm{t}, J=7.9 \mathrm{~Hz}, 1 \mathrm{H}), 6.96-6.91(\mathrm{~m}, 2 \mathrm{H})$, $6.47(\mathrm{~d}, J=2.4 \mathrm{~Hz}, 1 \mathrm{H}), 6.29$ (d, $J=8.1 \mathrm{~Hz}, 1 \mathrm{H}), 6.23$ (s, 1H), 2.32 (s, 3H), 1.32 (s, $9 \mathrm{H}) ;{ }^{13} \mathrm{C} \mathrm{NMR}\left(100 \mathrm{MHz}, \mathrm{CDCl}_{3}\right) \delta 146.9,139.2,139.0,136.2,133.5,131.5,129.4$, $128.9,128.8,128.2,127.8,125.4,123.8,119.5,111.7,96.7,90.5,58.3,23.2,17.7$. HRMS m/z calcd for $\mathrm{C}_{25} \mathrm{H}_{25} \mathrm{ClIN}_{2} \mathrm{OS}\left([\mathrm{M}+\mathrm{H}]^{+}\right)$: 563.0415, found 563.0410.

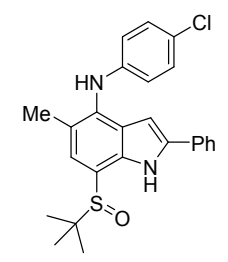

7-(tert-butylsulfinyl)- $N$-(4-chlorophenyl)-5-methyl-2-phenyl- $1 H$-indol-4-amine 10 : (eluent: petroleum ether/EtOAc 5:1), $25 \mathrm{mg}, 58 \%$; gray solid; m.p. $150-151{ }^{\circ} \mathrm{C} ;{ }^{1} \mathrm{H}$ NMR (400 MHz, $\left.\mathrm{CDCl}_{3}\right) \delta 10.54(\mathrm{~s}, 1 \mathrm{H}), 7.59(\mathrm{~d}, J=7.3 \mathrm{~Hz}, 2 \mathrm{H}), 7.39$ (t, $J=7.6 \mathrm{~Hz}$, 2H), 7.29 (t, $J=7.4 \mathrm{~Hz}, 1 \mathrm{H}), 7.17$ (s, $J=8.8 \mathrm{~Hz}, 2 \mathrm{H}), 6.89$ (s, 1H), 6.73 (d, $J=8.8 \mathrm{~Hz}$, 2H), $6.40(\mathrm{~d}, J=2.4 \mathrm{~Hz}, 1 \mathrm{H}), 5.75(\mathrm{~s}, 1 \mathrm{H}), 2.30(\mathrm{~s}, 3 \mathrm{H}), 1.31(\mathrm{~s}, 9 \mathrm{H}) ;{ }^{13} \mathrm{C}$ NMR $(100$ $\left.\mathrm{MHz}, \mathrm{CDCl}_{3}\right) \delta 143.0,138.5,136.3,134.3,131.6,128.9,128.0,125.9,125.3,124.5$, 124.1, 120.6, 117.4, 114.3, 97.0, 58.2, 23.2, 17.8. HRMS m/z calcd for $\mathrm{C}_{25} \mathrm{H}_{26} \mathrm{ClN}_{2} \mathrm{OS}$, $\left([\mathrm{M}+\mathrm{H}]^{+}\right): 437.1449$, found 437.1425 .

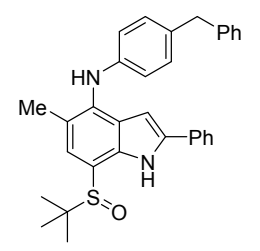

$\mathrm{N}$-(4-benzylphenyl)-7-(tert-butylsulfinyl)-5-methyl-2-phenyl-1 $H$-indol-4-amine 11 : (eluent: petroleum ether/EtOAc 5:1), $32 \mathrm{mg}, 65 \%$; gray solid; m.p. $155-156{ }^{\circ} \mathrm{C} ;{ }^{1} \mathrm{H}$ NMR (400 MHz, $\left.\mathrm{CDCl}_{3}\right) \delta 10.50(\mathrm{~s}, 1 \mathrm{H}), 7.56(\mathrm{~d}, J=7.4 \mathrm{~Hz}, 2 \mathrm{H}), 7.37$ (t, $J=7.3 \mathrm{~Hz}$, 2H), $7.28(\mathrm{t}, J=6.9 \mathrm{~Hz}, 3 \mathrm{H}), 7.20(\mathrm{~d}, J=7.5 \mathrm{~Hz}, 3 \mathrm{H}), 7.05(\mathrm{~d}, J=7.8 \mathrm{~Hz}, 2 \mathrm{H}), 6.88$ (d, $J=2.3 \mathrm{~Hz} 1 \mathrm{H}), 6.78$ (d, $J=7.7 \mathrm{~Hz}, 2 \mathrm{H}), 6.37$ (s, 1H), 5.73 (s, 1H), 3.93 (s, 2H), $2.30(\mathrm{~s}, 3 \mathrm{H}), 1.30(\mathrm{~s}, 9 \mathrm{H}) ;{ }^{13} \mathrm{C} \mathrm{NMR}\left(100 \mathrm{MHz}, \mathrm{CDCl}_{3}\right) \delta 142.33,141.5,137.9,136.4$, 
$135.5,133.1,131.8,129.4,128.8,128.8,128.4,127.8,125.9,125.3,125.2,124.3,119.4$, 117.4, 113.0, 97.4, 58.2, 41.2, 23.2, 17.8. HRMS m/z calcd for $\mathrm{C}_{32} \mathrm{H}_{33} \mathrm{~N}_{2} \mathrm{OS}\left([\mathrm{M}+\mathrm{H}]^{+}\right)$: 493.2308, found 493.2300 .

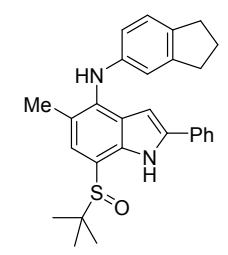

7-(tert-butylsulfinyl)- $\mathrm{N}$-(2,3-dihydro- $1 H$-inden-5-yl)-5-methyl-2-phenyl-1H-indol-

4-amine 12: (eluent: petroleum ether/EtOAc 5:1), $30 \mathrm{mg}$, 68\%; gray solid; m.p. 121$122{ }^{\circ} \mathrm{C} ;{ }^{1} \mathrm{H}$ NMR $\left(400 \mathrm{MHz}, \mathrm{CDCl}_{3}\right) \delta 10.49(\mathrm{~s}, 1 \mathrm{H}), 7.58(\mathrm{~d}, J=7.7 \mathrm{~Hz}, 2 \mathrm{H}), 7.37(\mathrm{t}$, $J=7.3 \mathrm{~Hz}, 2 \mathrm{H}), 7.31(\mathrm{t}, J=7.4 \mathrm{~Hz}, 2 \mathrm{H}), 7.08(\mathrm{~d}, J=8.1 \mathrm{~Hz}, 1 \mathrm{H}), 6.89$ (d, $J=2.2 \mathrm{~Hz}$, $1 \mathrm{H}), 6.75(\mathrm{~s}, 1 \mathrm{H}), 6.66(\mathrm{~d}, J=8.3 \mathrm{~Hz}, 1 \mathrm{H}), 6.42$ (s, 1H), 2.85 (q, $J=8.6 \mathrm{~Hz}, 4 \mathrm{H}), 2.31$ (s, 3H), 2.12-2.03 (m, 2H), 1.31 (s, 9H); $\left.{ }^{13} \mathrm{C} \mathrm{NMR} \mathrm{(100} \mathrm{MHz,} \mathrm{CDCl}_{3}\right) \delta$ 145.2, 142.6, $137.9,136.3,135.9,131.9,128.8,127.7,125.3,125.1,124.5,124.4,119.2,115.5,113.5$, 112.7, 97.4, 58.1, 33.0, 32.1, 25.7, 23.2, 17.8. HRMS m/z calcd for $\mathrm{C}_{28} \mathrm{H}_{31} \mathrm{~N}_{2} \mathrm{OS}$ $\left([\mathrm{M}+\mathrm{H}]^{+}\right):$443.2152, found 443.2144 .

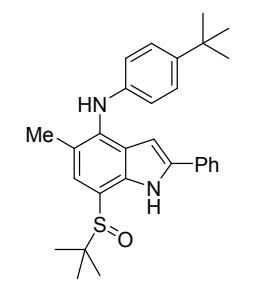

$\mathrm{N}$-(4-(tert-butyl)phenyl)-7-(tert-butylsulfinyl)-5-methyl-2-phenyl-1H-indol-4-

amine 13: (eluent: petroleum ether/EtOAc 5:1), $21 \mathrm{mg}$, 45\%; gray solid; m.p. 183-184 ${ }^{\circ} \mathrm{C} ;{ }^{1} \mathrm{H}$ NMR (400 MHz, $\left.\mathrm{CDCl}_{3}\right) \delta 10.50(\mathrm{~s}, 1 \mathrm{H}), 7.58(\mathrm{~d}, J=7.2 \mathrm{~Hz}, 2 \mathrm{H}),$,7.37 (t, $J=$ $7.6 \mathrm{~Hz}, 2 \mathrm{H}), 7.30-7.23(\mathrm{~m}, 4 \mathrm{H}), 6.89(\mathrm{~s}, 1 \mathrm{H}), 6.81(\mathrm{~d}, J=8.6 \mathrm{~Hz}, 2 \mathrm{H}), 6.38$ (d, $J=2.4$ $\mathrm{Hz}, 1 \mathrm{H}), 5.71(\mathrm{~s}, 1 \mathrm{H}), 2.31(\mathrm{~s}, 3 \mathrm{H}), 1.31(\mathrm{~s}, 18 \mathrm{H}) ;{ }^{13} \mathrm{C} \mathrm{NMR}\left(100 \mathrm{MHz}, \mathrm{CDCl}_{3}\right) \delta 143.3$, 141.7, 137.9, 136.5, 135.8, 131.9, 131.6, 128.8, 127.8, 125.8, 125.3, 124.3, 119.3, 117.0, $112.9,97.4,58.2,34.1,31.5,23.2,17.8$. HRMS m/z calcd for $\mathrm{C}_{29} \mathrm{H}_{35} \mathrm{~N}_{2} \mathrm{OS}\left([\mathrm{M}+\mathrm{H}]^{+}\right)$: 459.2465, found 459.2441 .

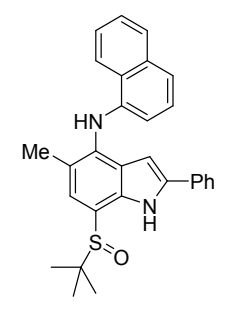




\section{7-(tert-butylsulfinyl)-5-methyl- $N$-(naphthalen-1-yl)-2-phenyl-1 H-indol-4-amine}

14: (eluent: petroleum ether/EtOAc 5:1), $18 \mathrm{mg}, 40 \%$; gray solid; m.p. $147-148{ }^{\circ} \mathrm{C} ;{ }^{1} \mathrm{H}$ NMR (400 MHz, $\left.\mathrm{CDCl}_{3}\right) \delta 10.53(\mathrm{~s}, 1 \mathrm{H}), 8.22(\mathrm{~d}, J=7.7 \mathrm{~Hz}, 1 \mathrm{H}), 7.90(\mathrm{~d}, J=6.9 \mathrm{~Hz}$, 2H), $7.54(\mathrm{ddd}, J=10.5,7.4,2.7 \mathrm{~Hz}, 4 \mathrm{H}), 7.48(\mathrm{~d}, J=8.1 \mathrm{~Hz}, 1 \mathrm{H}), 7.34(\mathrm{t}, J=7.4 \mathrm{~Hz}$, $3 \mathrm{H}), 7.26$ (q, $J=7.8 \mathrm{~Hz}, 3 \mathrm{H}), 6.94(\mathrm{~d}, J=2.3 \mathrm{~Hz}, 1 \mathrm{H}), 6.72(\mathrm{~d}, J=7.4 \mathrm{~Hz}, 2 \mathrm{H}), 6.32$ (s, 1H), 6.15 (s, 1H), 2.34 (s, 3H), 1.33 (s, 9H); $\left.{ }^{13} \mathrm{C} \mathrm{NMR} \mathrm{(100} \mathrm{MHz,} \mathrm{CDCl}_{3}\right) \delta 139.5$, $138.2,136.4,135.9,134.5,131.7,128.8,128.6,127.8,126.1,126.0,125.9,125.5,125.2$, 124.4, 121.1, 121.0, 119.5, 112.6, 113.3, 108.6, 97.0, 58.2, 23.2, 17.8. HRMS m/z calcd for $\mathrm{C}_{29} \mathrm{H}_{29} \mathrm{~N}_{2} \mathrm{OS}\left([\mathrm{M}+\mathrm{H}]^{+}\right)$: 453.1995, found 453.1982 .

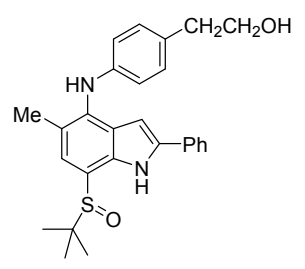

\section{2-(4-((7-(tert-butylsulfinyl)-5-methyl-2-phenyl-1 $H$-indol-4-}

yl)amino)phenyl)ethan-1-ol 15: (eluent: petroleum ether/EtOAc 5:1), $27 \mathrm{mg}, 60 \%$; gray solid; m.p. 178-179 ${ }^{\circ} \mathrm{C} ;{ }^{1} \mathrm{H}$ NMR $\left(400 \mathrm{MHz}, \mathrm{CDCl}_{3}\right) \delta 10.52(\mathrm{~s}, 1 \mathrm{H}), 7.60-7.56$ (m, 2H), 7.38 (t, $J=7.6 \mathrm{~Hz}, 2 \mathrm{H}), 7.29$ (dt, $J=8.1,1.6 \mathrm{~Hz}, 1 \mathrm{H}), 7.08$ (d, $J=8.4 \mathrm{~Hz}$, 2H), $6.90(\mathrm{~s}, 1 \mathrm{H}), 6.79(\mathrm{~d}, J=8.5 \mathrm{~Hz}, 2 \mathrm{H}), 6.41(\mathrm{~d}, J=2.5 \mathrm{~Hz}, 1 \mathrm{H}), 5.73(\mathrm{~s}, 1 \mathrm{H}), 3.84$ $(\mathrm{t}, J=6.6 \mathrm{~Hz}, 2 \mathrm{H}), 2.82(\mathrm{t}, J=6.6 \mathrm{~Hz}, 2 \mathrm{H}), 2.31(\mathrm{~s}, 3 \mathrm{H}), 1.31(\mathrm{~s}, 9 \mathrm{H}) ;{ }^{13} \mathrm{C}$ NMR $(100$ $\left.\mathrm{MHz}, \mathrm{CDCl}_{3}\right) \delta 142.9,138.1,136.4,135.2,131.8,129.9,129.6,128.9,127.9,125.6$, 125.3, 124.3, 112.0, 117.0, 113.5, 97.2, 63.8, 58.2, 38.4, 23.2, 17.8. HRMS m/z calcd for $\mathrm{C}_{27} \mathrm{H}_{31} \mathrm{~N}_{2} \mathrm{O}_{2} \mathrm{~S}\left([\mathrm{M}+\mathrm{H}]^{+}\right)$: 447.2101, found 447.2100.

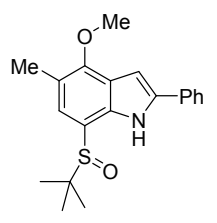

7-(tert-butylsulfinyl)-4-methoxy-5-methyl-2-phenyl-1H-indole $\quad$ 16: (eluent: petroleum ether/EtOAc 5:1), $29 \mathrm{mg}, 85 \%$; yellow solid; m.p. $138-139{ }^{\circ} \mathrm{C} ;{ }^{1} \mathrm{H}$ NMR $\left(400 \mathrm{MHz}, \mathrm{CDCl}_{3}\right) \delta 10.60(\mathrm{~s}, 1 \mathrm{H}), 7.68(\mathrm{~d}, J=7.2 \mathrm{~Hz}, 2 \mathrm{H}), 7.43(\mathrm{t}, J=7.9 \mathrm{~Hz}, 2 \mathrm{H})$, $7.32(\mathrm{t}, J=7.4 \mathrm{~Hz}, 1 \mathrm{H}), 6.89(\mathrm{~d}, J=2.5 \mathrm{~Hz}, 1 \mathrm{H}), 6.82(\mathrm{~s}, 1 \mathrm{H}), 4.14(\mathrm{~s}, 3 \mathrm{H}), 2.32$ (s, $3 \mathrm{H}), 1.28(\mathrm{~s}, 9 \mathrm{H}) ;{ }^{13} \mathrm{C} \mathrm{NMR}\left(100 \mathrm{MHz}, \mathrm{CDCl}_{3}\right) \delta 153.3,138.3,137.5,131.7,128.9$, 127.9, 125.3, 124.3, 123.0, 118.4, 112.6, 95.8, 60.1, 58.1, 23.1, 15.7. HRMS m/z calcd for $\mathrm{C}_{20} \mathrm{H}_{24} \mathrm{NO}_{2} \mathrm{~S}\left([\mathrm{M}+\mathrm{H}]^{+}\right)$: 342.1522 , found 342.1506. 


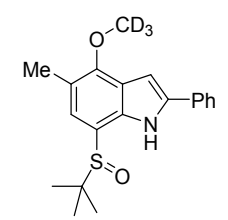

7-(tert-butylsulfinyl)-4-(methoxy-d3)-5-methyl-2-phenyl-1H-indole 17: (eluent: petroleum ether/EtOAc 5:1), $25 \mathrm{mg}, 72 \%$; yellow solid; m.p. 131-132 ${ }^{\circ} \mathrm{C} ;{ }^{1} \mathrm{H}$ NMR $\left(400 \mathrm{MHz}, \mathrm{CDCl}_{3}\right) \delta 10.60(\mathrm{~s}, 1 \mathrm{H}), 7.68(\mathrm{~d}, J=7.4 \mathrm{~Hz}, 3 \mathrm{H}), 7.43(\mathrm{t}, J=7.7 \mathrm{~Hz}, 3 \mathrm{H})$, $7.32(\mathrm{t}, J=7.4 \mathrm{~Hz}, 1 \mathrm{H}), 6.89(\mathrm{~d}, J=2.4 \mathrm{~Hz}, 1 \mathrm{H}), 6.82(\mathrm{~s}, 1 \mathrm{H}), 2.32(\mathrm{~s}, 3 \mathrm{H}), 1.28(\mathrm{~s}$, $9 \mathrm{H}) ;{ }^{13} \mathrm{C} \mathrm{NMR}\left(100 \mathrm{MHz}, \mathrm{CDCl}_{3}\right) \delta 153.3,138.3,137.5,131.7,128.9,127.9,125.3$, 124.3, 122.9, 118.4, 112.5, 95.8, 58.1, 23.1, 15.8. HRMS m/z calcd for $\mathrm{C}_{20} \mathrm{H}_{21} \mathrm{D}_{3} \mathrm{NO}_{2} \mathrm{~S}$ $\left([\mathrm{M}+\mathrm{H}]^{+}\right): 345.1711$, found 345.1700 .

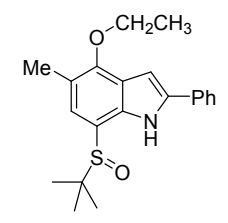

7-(tert-butylsulfinyl)-4-ethoxy-5-methyl-2-phenyl-1H-indole 18: (eluent: petroleum ether/EtOAc 5:1), $27 \mathrm{mg}, 76 \%$; yellow solid; m.p. 92-93 ${ }^{\circ} \mathrm{C}$; ${ }^{1} \mathrm{H}$ NMR (400 MHz, $\left.\mathrm{CDCl}_{3}\right) \delta 10.57(\mathrm{~s}, 1 \mathrm{H}), 7.68(\mathrm{~d}, J=8.1 \mathrm{~Hz}, 2 \mathrm{H}), 7.43(\mathrm{t}, J=7.6 \mathrm{~Hz}, 2 \mathrm{H}), 7.32(\mathrm{t}, J=$ $7.9 \mathrm{~Hz}, 1 \mathrm{H}), 6.82$ (s, 2H), 4.38 (q, $J=7.0 \mathrm{~Hz}, 2 \mathrm{H}), 2.33$ (s, 3H), 1.48 (t, $J=7.0 \mathrm{~Hz}$, 3H), $1.28(\mathrm{~s}, 9 \mathrm{H}) ;{ }^{13} \mathrm{C} \mathrm{NMR}\left(100 \mathrm{MHz}, \mathrm{CDCl}_{3}\right) \delta 138.3,137.3,131.8,128.9,127.9$, 125.3, 124.3, 123.7, 118.9, 112.7, 95.9, 68.3, 58.1, 23.1, 16.0, 15.9. HRMS m/z calcd for $\mathrm{C}_{21} \mathrm{H}_{26} \mathrm{NO}_{2} \mathrm{~S}\left([\mathrm{M}+\mathrm{H}]^{+}\right)$: 356.1679 , found 356.1652 .

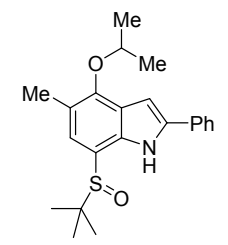

7-(tert-butylsulfinyl)-4-isopropoxy-5-methyl-2-phenyl-1H-indole $\quad$ 19: $\quad$ (eluent: petroleum ether/EtOAc 5:1), $24 \mathrm{mg}, 65 \%$; yellow solid; m.p. 165-166 ${ }^{\circ} \mathrm{C} ;{ }^{1} \mathrm{H}$ NMR $\left(400 \mathrm{MHz}, \mathrm{CDCl}_{3}\right) \delta 10.54(\mathrm{~s}, 1 \mathrm{H}), 7.68(\mathrm{~d}, J=6.9 \mathrm{~Hz}, 2 \mathrm{H}), 7.43(\mathrm{t}, J=7.7 \mathrm{~Hz}, 2 \mathrm{H})$, 7.32 (t, $J=7.4 \mathrm{~Hz}, 1 \mathrm{H}), 6.83(\mathrm{~s}, 1 \mathrm{H}), 6.77$ (d, $J=2.5 \mathrm{~Hz}, 1 \mathrm{H}), 4.79$ (hept, $J=6.1 \mathrm{~Hz}$, 1H), 2.33 (s, 3H), 1.40 (dd, $J=9.5,6.1 \mathrm{~Hz}, 6 \mathrm{H}), 1.28$ (s, 9H). ${ }^{13} \mathrm{C}$ NMR (100 MHz, $\left.\mathrm{CDCl}_{3}\right) \delta 151.5,138.2,137.1,131.8,128.9,127.9,125.3,124.7,124.3,120.0,113.0$, 96.0, 74.7, 58.1, 23.1, 23.0, 22.9, 16.3. HRMS m/z calcd for $\mathrm{C}_{22} \mathrm{H}_{28} \mathrm{NO}_{2} \mathrm{~S}\left([\mathrm{M}+\mathrm{H}]^{+}\right)$: 370.1835 , found 370.1847 . 


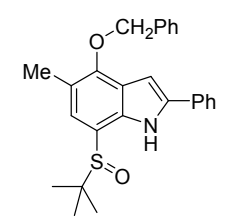

4-(benzyloxy)-7-(tert-butylsulfinyl)-5-methyl-2-phenyl-1H-indole 20: (eluent: petroleum ether/EtOAc 5:1), $25 \mathrm{mg}, 60 \%$; yellow solid; m.p. 127-128 ${ }^{\circ} \mathrm{C} ;{ }^{1} \mathrm{H}$ NMR $\left(400 \mathrm{MHz}, \mathrm{CDCl}_{3}\right) \delta 10.60(\mathrm{~s}, 1 \mathrm{H}), 7.67(\mathrm{~d}, J=7.4 \mathrm{~Hz}, 2 \mathrm{H}), 7.50(\mathrm{~d}, J=7.1 \mathrm{~Hz}, 2 \mathrm{H})$, $7.47-7.29$ (m, 7H), 5.33 (s, 2H), 2.29 (s, 3H), 1.29 (s, 9H); ${ }^{13} \mathrm{C}$ NMR (100 MHz, $\left.\mathrm{CDCl}_{3}\right) \delta 152.2,138.5,137.7,137.3,131.7,128.9,128.5,128.0,128.0,127.8,125.4$, 124.2, 123.8, 119.4, 113.3, 95.8, 74.5, 58.1, 23.1, 16.0. HRMS m/z calcd for $\mathrm{C}_{26} \mathrm{H}_{28} \mathrm{NO}_{2} \mathrm{~S}\left([\mathrm{M}+\mathrm{H}]^{+}\right): 418.1835$, found 418.1820 .

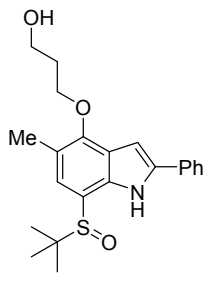

\section{3-((7-(tert-butylsulfinyl)-5-methyl-2-phenyl-1H-indol-4-yl)oxy)propan-1-ol 21 :}

(eluent: petroleum ether/EtOAc 3:1), 26 mg, 68\%; yellow oil; ${ }^{1} \mathrm{H}$ NMR (400 MHz, $\left.\mathrm{CDCl}_{3}\right) \delta 10.57(\mathrm{~s}, 1 \mathrm{H}), 7.69(\mathrm{~d}, J=7.3 \mathrm{~Hz}, 2 \mathrm{H}), 7.44(\mathrm{t}, J=7.6 \mathrm{~Hz}, 2 \mathrm{H}), 7.33(\mathrm{t}, J=$ $7.4 \mathrm{~Hz}, 1 \mathrm{H}), 6.86(\mathrm{~s}, J=2.4 \mathrm{~Hz}, 1 \mathrm{H}), 6.81(\mathrm{~s}, 1 \mathrm{H}), 4.47(\mathrm{t}, J=5.9 \mathrm{~Hz}, 2 \mathrm{H}), 4.01(\mathrm{t}, J=$ $5.9 \mathrm{~Hz}, 2 \mathrm{H}), 3.73(\mathrm{~s}, 1 \mathrm{H}), 2.32(\mathrm{~s}, 3 \mathrm{H}), 4.47(\mathrm{t}, J=5.9 \mathrm{~Hz}, 2 \mathrm{H}), 1.27(\mathrm{~s}, 9 \mathrm{H}) ;{ }^{13} \mathrm{C} \mathrm{NMR}$ $\left(100 \mathrm{MHz}, \mathrm{CDCl}_{3}\right) \delta 152.3,138.5,137.4,131.6,128.9,128.0$, , 125.3, 124.3, 123.4, 118.6, 112.9, 95.8, 70.7, 60.7, 58.2, 33.1, 23.1, 15.9. HRMS m/z calcd for $\mathrm{C}_{22} \mathrm{H}_{27} \mathrm{NNaO}_{3} \mathrm{~S}\left([\mathrm{M}+\mathrm{Na}]^{+}\right)$: 408.1604, found 408.1585.

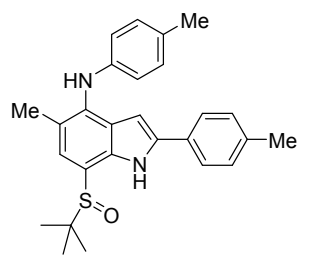

7-(tert-butylsulfinyl)-5-methyl- $N, 2$-di-p-tolyl-1 $H$-indol-4-amine $\quad 22$ : (eluent: petroleum ether/EtOAc 5:1), $32 \mathrm{mg}, 75 \%$; gray solid; m.p. 174-175 ${ }^{\circ} \mathrm{C} ;{ }^{1} \mathrm{H}$ NMR (400 $\left.\mathrm{MHz}, \mathrm{CDCl}_{3}\right) \delta 10.45(\mathrm{~s}, 1 \mathrm{H}), 7.47(\mathrm{~d}, J=7.9 \mathrm{~Hz}, 2 \mathrm{H}), 7.18(\mathrm{~d}, J=7.9 \mathrm{~Hz}, 2 \mathrm{H}), 7.04$ $(\mathrm{d}, J=8.0 \mathrm{~Hz}, 2 \mathrm{H}), 6.87(\mathrm{~s}, 1 \mathrm{H}), 6.76(\mathrm{~d}, J=8.2 \mathrm{~Hz}, 2 \mathrm{H}), 6.34(\mathrm{~d}, J=2.4 \mathrm{~Hz}, 1 \mathrm{H}), 5.68$ (s, 1H), $2.35(\mathrm{~s}, 3 \mathrm{H}), 2.31(\mathrm{~s}, 3 \mathrm{H}), 2.30(\mathrm{~s}, 3 \mathrm{H}), 1.31(\mathrm{~s}, 9 \mathrm{H}) ;{ }^{13} \mathrm{C}$ NMR (100 MHz, $\left.\mathrm{CDCl}_{3}\right) \delta 141.8,138.1,137.7,136.3,135.5,133.8,129.7,129.5,129.0,125.2,124.9$, 
119.2, 117.3, 112.8, 96.8, 58.1, 23.2, 21.2, 20.6, 17.8. HRMS m/z calcd for $\mathrm{C}_{27} \mathrm{H}_{31} \mathrm{~N}_{2} \mathrm{OS}\left([\mathrm{M}+\mathrm{H}]^{+}\right)$: 431.2152, found 431.2131.

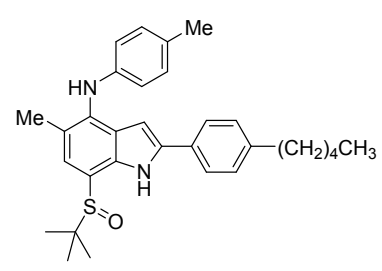

7-(tert-butylsulfinyl)-5-methyl-2-(4-pentylphenyl)- $\mathrm{N}$-(p-tolyl)- $1 \mathrm{H}$-indol-4-amine

23: (eluent: petroleum ether/EtOAc 5:1), $23 \mathrm{mg}, 48 \%$; gray solid; m.p. $133-134{ }^{\circ} \mathrm{C} ;{ }^{1} \mathrm{H}$ NMR (400 MHz, $\left.\mathrm{CDCl}_{3}\right) \delta 10.45(\mathrm{~s}, 1 \mathrm{H}), 7.49(\mathrm{~d}, J=7.9 \mathrm{~Hz}, 2 \mathrm{H}), 7.18(\mathrm{~d}, J=7.9 \mathrm{~Hz}$, 2H), 7.04 (d, $J=8.0 \mathrm{~Hz}, 2 \mathrm{H}), 6.87$ (s, 1H), 6.77 (d, $J=8.3 \mathrm{~Hz}, 2 \mathrm{H}), 6.34$ (d, $J=2.5$ $\mathrm{Hz}, 1 \mathrm{H}), 5.68(\mathrm{~s}, 1 \mathrm{H}), 2.60(\mathrm{t}, J=7.7 \mathrm{~Hz}, 2 \mathrm{H}), 2.30(\mathrm{~s}, 6 \mathrm{H}), 1.62$ (q, $J=7.5 \mathrm{~Hz}, 2 \mathrm{H})$, $1.32-1.30(\mathrm{~m}, 4 \mathrm{H}), 1.30(\mathrm{~s}, 9 \mathrm{H}), 0.89(\mathrm{t}, J=6.7 \mathrm{~Hz}, 3 \mathrm{H}) ;{ }^{13} \mathrm{C} \mathrm{NMR}\left(100 \mathrm{MHz}, \mathrm{CDCl}_{3}\right)$ $\delta 142.8,141.8,138.1,136.3,135.5,135.0,129.7,129.5,129.2,128.9,125.2,124.1$, 119.1, 117.3, 112.8, 96.8, 58.1, 35.6, 31.4, 31.0, 23.2, 22.5, 20.6, 17.8, 14.0. HRMS $\mathrm{m} / \mathrm{z}$ calcd for $\mathrm{C}_{31} \mathrm{H}_{39} \mathrm{~N}_{2} \mathrm{OS}\left([\mathrm{M}+\mathrm{H}]^{+}\right)$: 487.2778, found 487.2758 .

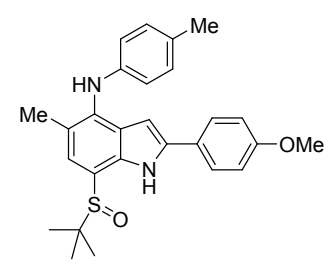

\section{7-(tert-butylsulfinyl)-2-(4-methoxyphenyl)-5-methyl- $N$-(p-tolyl)-1H-indol-4-}

amine 24: (eluent: petroleum ether/EtOAc 5:1), $25 \mathrm{mg}, 56 \%$; gray solid; m.p. 159$160{ }^{\circ} \mathrm{C} ;{ }^{1} \mathrm{H}$ NMR $\left(400 \mathrm{MHz}, \mathrm{CDCl}_{3}\right) \delta 10.40(\mathrm{~s}, 1 \mathrm{H}), 7.51(\mathrm{~d}, J=8.8 \mathrm{~Hz}, 2 \mathrm{H}), 7.04(\mathrm{~d}$, $J=8.0 \mathrm{~Hz}, 2 \mathrm{H}), 6.91(\mathrm{~d}, J=8.8 \mathrm{~Hz}, 2 \mathrm{H}), 6.86(\mathrm{~s}, 1 \mathrm{H}), 6.77(\mathrm{~d}, J=8.4 \mathrm{~Hz}, 2 \mathrm{H}), 6.27$ $(\mathrm{d}, J=2.5 \mathrm{~Hz}, 2 \mathrm{H}), 5.67(\mathrm{~s}, 1 \mathrm{H}), 3.83(\mathrm{~s}, 3 \mathrm{H}), 2.30(\mathrm{~s}, 3 \mathrm{H}), 2.30(\mathrm{~s}, 3 \mathrm{H}), 1.31(\mathrm{~s}, 9 \mathrm{H})$. ${ }^{13} \mathrm{C} \mathrm{NMR}\left(100 \mathrm{MHz}, \mathrm{CDCl}_{3}\right) \delta 159.3,141.7,137.9,136.2$, 135.3, 129.5, 129.4, 126.6, 125.3, 124.6, 123.7, 119.2, 117.1, 114.2, 112.7, 96.1, 58.0, 55.2, 23.1, 20.5, 17.7. HRMS m/z calcd for $\mathrm{C}_{27} \mathrm{H}_{31} \mathrm{~N}_{2} \mathrm{O}_{2} \mathrm{~S}\left([\mathrm{M}+\mathrm{H}]^{+}\right)$: 447.2101, found 447.2103.

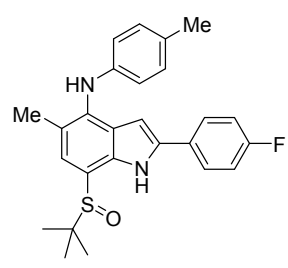

\section{7-(tert-butylsulfinyl)-2-(4-fluorophenyl)-5-methyl- $N$-(p-tolyl)-1 $H$-indol-4-amine}

25: (eluent: petroleum ether/EtOAc 5:1), $22 \mathrm{mg}, 50 \%$; gray solid; m.p. 172-173 ${ }^{\circ} \mathrm{C}$; 
${ }^{1} \mathrm{H}$ NMR (400 MHz, $\left.\mathrm{CDCl}_{3}\right) \delta 10.47(\mathrm{~s}, 1 \mathrm{H}), 7.57$ - 7.49 (m, 2H), 7.10 - $7.01(\mathrm{~m}, 4 \mathrm{H})$, $6.88(\mathrm{~s}, 1 \mathrm{H}), 6.80-6.75(\mathrm{~m}, 2 \mathrm{H}), 6.30(\mathrm{~d}, J=2.5 \mathrm{~Hz}, 1 \mathrm{H}), 5.69(\mathrm{~s}, 1 \mathrm{H}), 2.31(\mathrm{~s}, 3 \mathrm{H}), 2.30$ (s, 3H), $1.31(\mathrm{~s}, 9 \mathrm{H}) ;{ }^{13} \mathrm{C}$ NMR $\left(100 \mathrm{MHz}, \mathrm{CDCl}_{3}\right) \delta 162.4(\mathrm{~d}, J(\mathrm{C} . \mathrm{F})=247.7 \mathrm{~Hz})$, 141.6, 137.0, 136.5, 135.7, 129.9, 129.5, $128.2(\mathrm{~d}, J(\mathrm{C} . \mathrm{F})=2.7 \mathrm{~Hz}), 127.0(\mathrm{~d}, J(\mathrm{C} . \mathrm{F})=$ 8.1 Hz), 125.0, 124.4, 119.1, 117.5, 115.9 (d, J(C.F) = 21.9 Hz)., 112.7, 97.3, 58.2, 23.2, 20.6, 17.8. HRMS m/z calcd for $\mathrm{C}_{26} \mathrm{H}_{28} \mathrm{FN}_{2} \mathrm{OS}\left([\mathrm{M}+\mathrm{H}]^{+}\right)$: 435.1901, found 435.1908 .

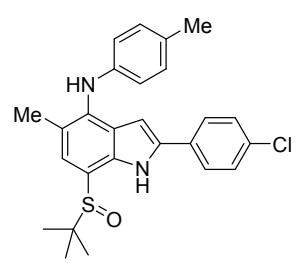

\section{7-(tert-butylsulfinyl)-2-(4-chlorophenyl)-5-methyl- $N$-(p-tolyl)-1H-indol-4-amine}

26: (eluent: petroleum ether/EtOAc 5:1), $31 \mathrm{mg}, 68 \%$; gray solid; m.p. 178-179 ${ }^{\circ} \mathrm{C}$; ${ }^{1} \mathrm{H}$ NMR (400 MHz, $\left.\mathrm{CDCl}_{3}\right) \delta 10.50(\mathrm{~s}, 1 \mathrm{H}), 7.48(\mathrm{~d}, J=8.5 \mathrm{~Hz}, 2 \mathrm{H}), 7.33(\mathrm{~d}, J=8.5$ Hz, 2H)., $7.04(\mathrm{~d}, J=8.0 \mathrm{~Hz}, 2 \mathrm{H}), 6.89$ (s, 1H), 6.77 (d, $J=8.3 \mathrm{~Hz}, 2 \mathrm{H}), 6.34$ (d, $J=$ $2.5 \mathrm{~Hz}, 1 \mathrm{H}), 5.70(\mathrm{~s}, 1 \mathrm{H}), 2.30(\mathrm{~s}, 6 \mathrm{H}), 1.31(\mathrm{~s}, 9 \mathrm{H}) ;{ }^{13} \mathrm{C} \mathrm{NMR}\left(100 \mathrm{MHz}, \mathrm{CDCl}_{3}\right) \delta$ 141.5, 136.6, 135.9, 133.4, 130.3, 130.0, 129.5, 129.1, 129.0, 126.4, 124.8, 124.6, 119.1, 117.5, 112.6, 97.9, 58.2, 23.2, 20.6, 17.8. HRMS m/z calcd for $\mathrm{C}_{26} \mathrm{H}_{28} \mathrm{ClN}_{2} \mathrm{OS}$ $\left([\mathrm{M}+\mathrm{H}]^{+}\right): 451.1605$, found 451.1597 .

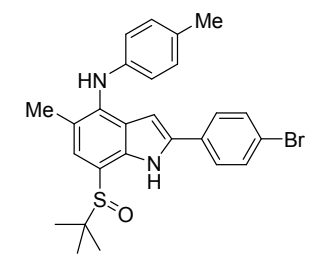

\section{2-(4-bromophenyl)-7-(tert-butylsulfinyl)-5-methyl- $\mathrm{N}$-( $p$-tolyl)-1H-indol-4-amine}

27: (eluent: petroleum ether/EtOAc 5:1), $27 \mathrm{mg}, 55 \%$; gray solid; m.p. 169-170 ${ }^{\circ} \mathrm{C}$; ${ }^{1} \mathrm{H}$ NMR (400 MHz, $\left.\mathrm{CDCl}_{3}\right) \delta 10.51(\mathrm{~s}, 1 \mathrm{H}), 7.48(\mathrm{~d}, J=8.4 \mathrm{~Hz}, 2 \mathrm{H}), 7.41(\mathrm{~d}, J=8.5$ $\mathrm{Hz}, 2 \mathrm{H}), 7.26$ (s, 1H), 7.05 (d, $J=8.1 \mathrm{~Hz}, 2 \mathrm{H}), 6.89$ (s, 1H), $6.77(\mathrm{~d}, J=8.2 \mathrm{~Hz}, 2 \mathrm{H})$, $6.35(\mathrm{~d}, J=2.4 \mathrm{~Hz}, 1 \mathrm{H}), 5.70(\mathrm{~s}, 1 \mathrm{H}), 2.31(\mathrm{~s}, 6 \mathrm{H}), 1.31(\mathrm{~s}, 9 \mathrm{H}) ;{ }^{13} \mathrm{C}$ NMR $(100 \mathrm{MHz}$, $\left.\mathrm{CDCl}_{3}\right) \delta 141.5,136.6,135.9,131.9,130.8,130.0,129.5,127.4,126.7,124.8,124.7$, 121.6, 119.1, 117.6, 112.7, 97.9, 58.2, 23.2, 20.6, 17.8. HRMS m/z calcd for $\mathrm{C}_{26} \mathrm{H}_{28} \mathrm{BrN}_{2} \mathrm{OS}\left([\mathrm{M}+\mathrm{H}]^{+}\right)$: 495.1100, found 495.1077. 


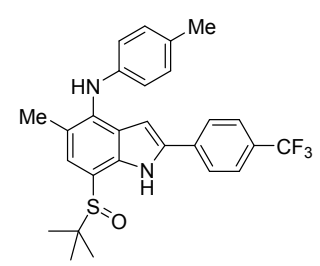

7-(tert-butylsulfinyl)-5-methyl- $N$-(p-tolyl)-2-(4-(trifluoromethyl)phenyl)-1H-

indol-4-amine 28: (eluent: petroleum ether/EtOAc 5:1), $33 \mathrm{mg}, 68 \%$; gray solid; m.p. 161-162 ${ }^{\circ} \mathrm{C} ;{ }^{1} \mathrm{H}$ NMR (400 MHz, $\left.\mathrm{CDCl}_{3}\right) \delta 10.62(\mathrm{~s}, 1 \mathrm{H}), 7.62(\mathrm{q}, J=8.5 \mathrm{~Hz}, 4 \mathrm{H}), 7.06$ $(\mathrm{d}, J=8.1 \mathrm{~Hz}, 2 \mathrm{H}), 6.92(\mathrm{~s}, 1 \mathrm{H}), 6.80(\mathrm{~d}, J=8.3 \mathrm{~Hz}, 2 \mathrm{H}), 6.43(\mathrm{~d}, J=2.5 \mathrm{~Hz}, 1 \mathrm{H}), 5.73$ (s, 1H), $2.31(\mathrm{~s}, 6 \mathrm{H}), 1.31(\mathrm{~s}, 9 \mathrm{H}) \cdot{ }^{13} \mathrm{C} \mathrm{NMR}\left(100 \mathrm{MHz}, \mathrm{CDCl}_{3}\right) \delta 141.4,136.9,136.2$, 136.0, 135.2, 130.3, 129.5, $129.3(\mathrm{q}, J(\mathrm{C}, \mathrm{F})=32.8 \mathrm{~Hz}), 129.1,125.8,125.8,125.7$, 125.3, 125.2, 124.5, $124.0(\mathrm{q}, J(\mathrm{C}, \mathrm{F})=272.00 \mathrm{~Hz}), 118.9,117.8,112.5,99.1,58.2$, 23.1, 20.6, 17.8. HRMS m/z calcd for $\mathrm{C}_{27} \mathrm{H}_{28} \mathrm{~F}_{3} \mathrm{~N}_{2} \mathrm{OS}\left([\mathrm{M}+\mathrm{H}]^{+}\right)$: 485.1869, found 485.1860 .

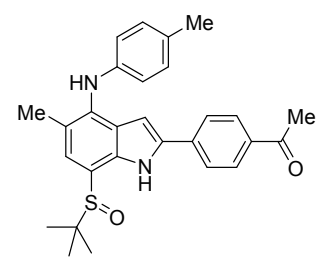

1-(4-(7-(tert-butylsulfinyl)-5-methyl-4-(p-tolylamino)-1H-indol-2-

yl)phenyl)ethan-1-one 29: (eluent: petroleum ether/EtOAc 5:1), $21 \mathrm{mg}, 45 \%$; yellow solid; m.p. 85-86 ${ }^{\circ} \mathrm{C} ;{ }^{1} \mathrm{H}$ NMR (400 MHz, $\left.\mathrm{CDCl}_{3}\right) \delta 10.64$ (s, 1H), 7.96 (d, J=8.6 Hz, 2H), $7.63(\mathrm{~d}, J=8.6 \mathrm{~Hz}, 2 \mathrm{H}), 7.06(\mathrm{~d}, J=8.0 \mathrm{~Hz}, 2 \mathrm{H}), 6.92(\mathrm{~s}, 1 \mathrm{H}), 6.80(\mathrm{~d}, J=8.4$ $\mathrm{Hz}, 2 \mathrm{H}), 6.48(\mathrm{~d}, J=2.5 \mathrm{~Hz}, 1 \mathrm{H}), 5.72(\mathrm{~s}, 1 \mathrm{H}), 2.60$ (s, 3H), 2.32 (s, 6H), 1.32 (s, 9H); ${ }^{13} \mathrm{C} \mathrm{NMR}\left(100 \mathrm{MHz}, \mathrm{CDCl}_{3}\right) \delta 191.9,141.8,138.1,137.7,136.3,135.5,133.8,129.7$, 129.5, 129.0, 125.2, 124.1, 119.2, 117.3, 112.8, 96.8, 58.1, 23.2, 21.2, 20.6, 17.8. HRMS m/z calcd for $\mathrm{C}_{28} \mathrm{H}_{31} \mathrm{~N}_{2} \mathrm{O}_{2} \mathrm{~S}\left([\mathrm{M}+\mathrm{H}]^{+}\right)$: 459.2101, found 459.2107.

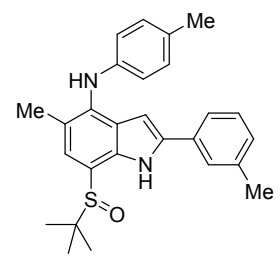

7-(tert-butylsulfinyl)-5-methyl-2-(m-tolyl)- $N$-(p-tolyl)-1H-indol-4-amine

30:

(eluent: petroleum ether/EtOAc 5:1), $25 \mathrm{mg}, 58 \%$; gray solid; m.p. $160-161{ }^{\circ} \mathrm{C} ;{ }^{1} \mathrm{H}$ NMR (400 MHz, $\left.\mathrm{CDCl}_{3}\right) \delta 10.47(\mathrm{~s}, 1 \mathrm{H}), 7.41-7.36(\mathrm{~m}, 2 \mathrm{H}), 7.26(\mathrm{t}, J=7.9 \mathrm{~Hz}, 2 \mathrm{H})$, $7.09(\mathrm{~d}, J=7.6 \mathrm{~Hz}, 1 \mathrm{H}), 7.04(\mathrm{~d}, J=8.1 \mathrm{~Hz}, 2 \mathrm{H}), 6.88(\mathrm{~s}, 1 \mathrm{H}), 6.76(\mathrm{~d}, J=8.4 \mathrm{~Hz}, 2 \mathrm{H})$, $6.38(\mathrm{~d}, J=2.5 \mathrm{~Hz}, 1 \mathrm{H}), 5.69(\mathrm{~s}, 1 \mathrm{H}), 2.37$ (s, 3H), 2.30 (s, 3H), 2.30 (s, 3H), 1.31 (s, 
9H); ${ }^{13} \mathrm{C} \mathrm{NMR}\left(100 \mathrm{MHz}, \mathrm{CDCl}_{3}\right) \delta 141.8,138.5,138.1,136.4,135.6,131.8,129.7$, $129.5,128.7,128.6,125.9,125.2,124.2,122.6,119.3,117.2,112.9,97.3,58.1,23.2$, 21.4, 20.6, 17.8. HRMS $\mathrm{m} / \mathrm{z}$ calcd for $\mathrm{C}_{27} \mathrm{H}_{31} \mathrm{~N}_{2} \mathrm{OS}\left([\mathrm{M}+\mathrm{H}]^{+}\right)$: 431.2152, found 431.2151 .

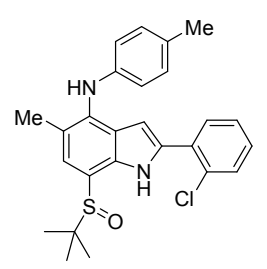

\section{7-(tert-butylsulfinyl)-2-(2-chlorophenyl)-5-methyl- $N$-(p-tolyl)-1H-indol-4-amine}

31: (eluent: petroleum ether/EtOAc 5:1), $23 \mathrm{mg}, 50 \%$; gray solid; m.p. 179-180 ${ }^{\circ} \mathrm{C}$; ${ }^{1} \mathrm{H}$ NMR (400 MHz, $\left.\mathrm{CDCl}_{3}\right) \delta 10.70(\mathrm{~s}, 1 \mathrm{H}), 7.51$ (dd, $\left.J=7.5,2.0 \mathrm{~Hz}, 1 \mathrm{H}\right), 7.43$ (dd, $J$ $=7.6,1.7 \mathrm{~Hz}, 1 \mathrm{H}), 7.30-7.17(\mathrm{~m}, 2 \mathrm{H}), 7.04(\mathrm{~d}, J=8.2 \mathrm{~Hz}, 2 \mathrm{H}), 6.93(\mathrm{~s}, 1 \mathrm{H}), 6.80(\mathrm{~d}$, $J=8.3 \mathrm{~Hz}, 2 \mathrm{H}), 6.41(\mathrm{~d}, J=2.5 \mathrm{~Hz}, 1 \mathrm{H}), 5.73(\mathrm{~s}, 1 \mathrm{H}), 2.31(\mathrm{~s}, 3 \mathrm{H}), 2.30(\mathrm{~s}, 3 \mathrm{H}), 1.31$ (s, 9H); ${ }^{13} \mathrm{C} \mathrm{NMR}\left(100 \mathrm{MHz}, \mathrm{CDCl}_{3}\right) \delta 141.5,136.1,135.7,134.9,131.6,130.7,130.6$, $130.1,129.5,128.8,127.0,124.9,123.8,120.7,118.6,117.8,101.2$, 58.0, 23.2 , 20.6, 17.8. HRMS m/z calcd for $\mathrm{C}_{26} \mathrm{H}_{28} \mathrm{ClN}_{2} \mathrm{OS}\left([\mathrm{M}+\mathrm{H}]^{+}\right)$: 451.1605 , found 451.1602 .

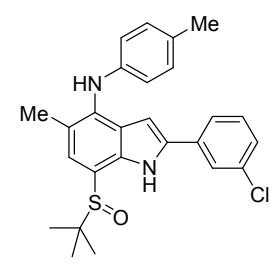

\section{7-(tert-butylsulfinyl)-2-(3-chlorophenyl)-5-methyl- $N$-(p-tolyl)-1H-indol-4-amine}

32: (eluent: petroleum ether/EtOAc 5:1), $16 \mathrm{mg}, 35 \%$; gray solid; m.p. 164-165 ${ }^{\circ} \mathrm{C}$; ${ }^{1} \mathrm{H}$ NMR $\left(400 \mathrm{MHz}, \mathrm{CDCl}_{3}\right) \delta 10.47(\mathrm{~s}, 1 \mathrm{H}), 7.44-7.36(\mathrm{~m}, 2 \mathrm{H}), 7.26(\mathrm{t}, J=7.7 \mathrm{~Hz}$, 1H), $7.09(\mathrm{~d}, J=7.6 \mathrm{~Hz}, 1 \mathrm{H}), 7.04(\mathrm{~d}, J=8.2 \mathrm{~Hz}, 2 \mathrm{H}), 6.88(\mathrm{~s}, 1 \mathrm{H}), 6.76(\mathrm{~d}, J=8.4$ $\mathrm{Hz}, 2 \mathrm{H}), 6.38(\mathrm{~d}, J=2.5 \mathrm{~Hz}, 1 \mathrm{H}), 5.69(\mathrm{~s}, 1 \mathrm{H}), 2.37$ (s, 3H), $2.30(\mathrm{~s}, 3 \mathrm{H}), 2.30(\mathrm{~s}, 3 \mathrm{H})$, $1.31(\mathrm{~s}, 9 \mathrm{H}) ;{ }^{13} \mathrm{C} \mathrm{NMR}\left(100 \mathrm{MHz}, \mathrm{CDCl}_{3}\right) \delta 141.5,136.7,136.3,136.0,134.8,133.7$, 130.1, 129.6, 127.6, 125.3, 124.8, 124.7, 123.3, 120.9, 119.1, 117.6, 112.7, 98.4, 58.2, 23.2, 20.6, 17.8. HRMS m/z calcd for $\mathrm{C}_{26} \mathrm{H}_{28} \mathrm{ClN}_{2} \mathrm{OS}\left([\mathrm{M}+\mathrm{H}]^{+}\right): 451.1605$, found 451.1602 .

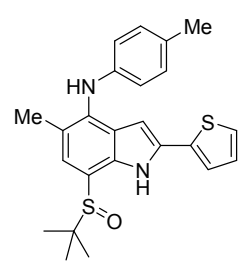


7-(tert-butylsulfinyl)-5-methyl-2-(thiophen-2-yl)- $N$-(p-tolyl)-1H-indol-4-amine 33 : (eluent: petroleum ether/EtOAc 5:1), $22 \mathrm{mg}, 51 \%$; gray solid; m.p. $180-181{ }^{\circ} \mathrm{C} ;{ }^{1} \mathrm{H}$ NMR (400 MHz, $\left.\mathrm{CDCl}_{3}\right) \delta 10.36(\mathrm{~s}, 1 \mathrm{H}), 7.24-7.19(\mathrm{~m}, 2 \mathrm{H}), 7.06-7.01(\mathrm{~m}, 3 \mathrm{H})$, $6.87(\mathrm{~s}, 1 \mathrm{H}), 6.75$ (d, $J=8.4 \mathrm{~Hz}, 2 \mathrm{H}), 6.28$ (d, $J=2.4 \mathrm{~Hz}, 1 \mathrm{H}), 5.68(\mathrm{~s}, 1 \mathrm{H}), 2.30$ (s, 3H), 2.29 (s, 3H), $1.30(\mathrm{~s}, 9 \mathrm{H}) ;{ }^{13} \mathrm{C} \mathrm{NMR}\left(100 \mathrm{MHz}, \mathrm{CDCl}_{3}\right) \delta 141.6,136.1,135.6$, 135.2, 132.4, 129.8, 129.5, 127.8, 125.0, 124.8, 124.4, 123.4, 119.5, 117.3, 112.8, 97.8, 58.1, 23.2, 20.6, 17.8. HRMS m/z calcd for $\mathrm{C}_{24} \mathrm{H}_{27} \mathrm{~N}_{2} \mathrm{OS}_{2}\left([\mathrm{M}+\mathrm{H}]^{+}\right)$: 423.1559, found 423.1550 .

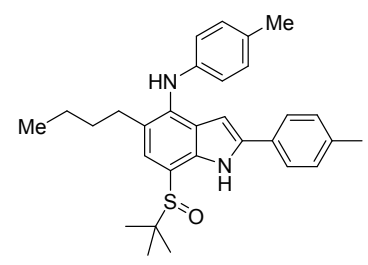

5-butyl-7-(tert-butylsulfinyl)- $N, 2-d i-p-t o l y l-1 H$-indol-4-amine $\quad 34: \quad$ (eluent: petroleum ether/EtOAc 5:1), $26 \mathrm{mg}, 56 \%$; gray solid; m.p. $98-99{ }^{\circ} \mathrm{C} ;{ }^{1} \mathrm{H}$ NMR (400 $\left.\mathrm{MHz}, \mathrm{CDCl}_{3}\right) \delta 10.43(\mathrm{~s}, 1 \mathrm{H}), 7.46(\mathrm{~d}, J=7.9 \mathrm{~Hz}, 2 \mathrm{H}), 7.17(\mathrm{~d}, J=7.8 \mathrm{~Hz}, 2 \mathrm{H}), 7.02$ $(\mathrm{d}, J=8.1 \mathrm{~Hz}, 1 \mathrm{H}), 6.87(\mathrm{~s}, 1 \mathrm{H}), 6.74(\mathrm{~d}, J=8.3 \mathrm{~Hz}, 2 \mathrm{H}), 6.31(\mathrm{~d}, J=2.0 \mathrm{~Hz}, 1 \mathrm{H}), 5.69$ (s, 1H), $2.66(\mathrm{td}, J=7.4,2.9 \mathrm{~Hz}, 2 \mathrm{H}), 2.35$ (s, 3H), 2.29 (s, 3H), 1.57 (t, $J=7.6 \mathrm{~Hz}$, 2H), $1.38-1.33(\mathrm{~m}, 2 \mathrm{H}), 1.30(\mathrm{~s}, 9 \mathrm{H}), 0.89(\mathrm{t}, J=7.3 \mathrm{~Hz}, 3 \mathrm{H}) ;{ }^{13} \mathrm{C}$ NMR $(100 \mathrm{MHz}$, $\left.\mathrm{CDCl}_{3}\right) \delta 142.3,138.1,137.7,136.1,135.0,129.5,129.1,125.8,125.3,124.6,123.3$, 117.0, 113.3, 97.2, 58.2, 32.8, 31.0, 23.2, 22.4, 21.2, 20.6, 13.9. HRMS m/z calcd for $\mathrm{C}_{30} \mathrm{H}_{37} \mathrm{~N}_{2} \mathrm{OS}\left([\mathrm{M}+\mathrm{H}]^{+}\right)$: 473.2621, found 473.2616.

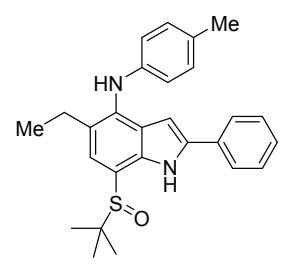

7-(tert-butylsulfinyl)-5-ethyl-2-phenyl- $N$-(p-tolyl)-1H-indol-4-amine 35 : (eluent: petroleum ether/EtOAc 5:1), $30 \mathrm{mg}, 69 \%$; gray solid; m.p. 165-166 ${ }^{\circ} \mathrm{C} ;{ }^{1} \mathrm{H}$ NMR (400 $\left.\mathrm{MHz}, \mathrm{CDCl}_{3}\right) \delta 10.50(\mathrm{~s}, 1 \mathrm{H}), 7.51(\mathrm{~d}, J=7.2 \mathrm{~Hz}, 2 \mathrm{H}), 7.39$ (t, $\left.J=7.6 \mathrm{~Hz}, 2 \mathrm{H}\right), 7.29$ $(\mathrm{d}, J=8.3 \mathrm{~Hz}, 2 \mathrm{H}), 7.03(\mathrm{~d}, J=8.1 \mathrm{~Hz}, 2 \mathrm{H}), 6.91(\mathrm{~s}, 1 \mathrm{H}), 6.77(\mathrm{~d}, J=8.4 \mathrm{~Hz}, 2 \mathrm{H}), 6.38$ (d, $J=2.5 \mathrm{~Hz}, 1 \mathrm{H}), 5.71(\mathrm{~s}, 1 \mathrm{H}), 2.72(\mathrm{q}, J=7.5 \mathrm{~Hz}, 2 \mathrm{H}), 2.29(\mathrm{~s}, 3 \mathrm{H}), 1.31$ (s, 9H), $1.24(\mathrm{t}, J=7.5 \mathrm{~Hz}, 3 \mathrm{H}) .{ }^{13} \mathrm{C} \mathrm{NMR}\left(100 \mathrm{MHz}, \mathrm{CDCl}_{3}\right) \delta 142.3,138.0,136.3,135.0$, $131.9,129.5,129.1,128.8,127.8,126.1,125.8,125.3,122.7,117.0,113.5,97.7,58.2$, 
24.3, 23.2, 20.6, 15.0. HRMS m/z calcd for $\mathrm{C}_{27} \mathrm{H}_{31} \mathrm{~N}_{2} \mathrm{OS}\left([\mathrm{M}+\mathrm{H}]^{+}\right): 431.2152$, found 431.2147 .

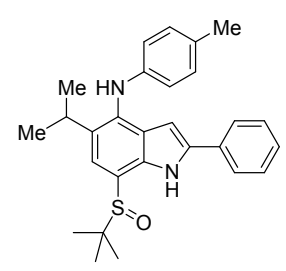

7-(tert-butylsulfinyl)-5-isopropyl-2-phenyl- $N$-(p-tolyl)- $1 H$-indol-4-amine

36:

(eluent: petroleum ether/EtOAc 5:1), $18 \mathrm{mg}, 41 \%$; gray solid; m.p. 167-168 ${ }^{\circ} \mathrm{C} ;{ }^{1} \mathrm{H}$ NMR (400 MHz, $\left.\mathrm{CDCl}_{3}\right) \delta 10.48(\mathrm{~s}, 1 \mathrm{H}), 7.59(\mathrm{~d}, J=8.5 \mathrm{~Hz}, 2 \mathrm{H}), 7.39$ (t, $J=7.6 \mathrm{~Hz}$, 2H), $7.30(\mathrm{~d}, J=8.3 \mathrm{~Hz}, 2 \mathrm{H}), 7.02(\mathrm{~d}, J=8.2 \mathrm{~Hz}, 2 \mathrm{H}), 6.97(\mathrm{~s}, 1 \mathrm{H}), 6.72(\mathrm{~d}, J=8.4 \mathrm{~Hz}$, $1 \mathrm{H}), 6.41(\mathrm{~d}, J=2.5 \mathrm{~Hz}, 1 \mathrm{H}), 5.67(\mathrm{~s}, 1 \mathrm{H}), 3.33$ (hept, $J=6.8 \mathrm{~Hz}, 1 \mathrm{H}), 2.28(\mathrm{~s}, 3 \mathrm{H})$, $1.30(\mathrm{~s}, 9 \mathrm{H}), 1.24(\mathrm{~d}, J=3.5 \mathrm{~Hz}, 6 \mathrm{H}) .{ }^{13} \mathrm{C} \mathrm{NMR}\left(100 \mathrm{MHz}, \mathrm{CDCl}_{3}\right) \delta 143.0,138.2$, 135.9, 133.9, 131.9, 129.9, 129.6, 129.0, 128.8, 126.6, 125.4, 119.6, 116.3, 114.6, 97.7, 58.1, 27.5, 27.2, 23.8, 23.7, 23.2. HRMS m/z calcd for $\mathrm{C}_{28} \mathrm{H}_{33} \mathrm{~N}_{2} \mathrm{OS}\left([\mathrm{M}+\mathrm{H}]^{+}\right)$: 445.2308, found 445.2251.

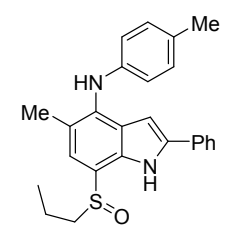

5-methyl-2-phenyl-7-(propylsulfinyl)- $N$-(p-tolyl)-1H-indol-4-amine 37 : (eluent: petroleum ether/EtOAc 5:1), $18 \mathrm{mg}, 45 \%$; gray oil. ${ }^{1} \mathrm{H}$ NMR (400 $\left.\mathrm{MHz}, \mathrm{CDCl}_{3}\right) \delta$ 10.29 (s, 1H), $7.61(\mathrm{~d}, J=7.6 \mathrm{~Hz}, 2 \mathrm{H}), 7.40$ (t, $J=7.4 \mathrm{~Hz}, 2 \mathrm{H}), 7.30$ (t, $J=7.5 \mathrm{~Hz}, 1 \mathrm{H})$, 7.05 (d, $J=7.3 \mathrm{~Hz}, 2 \mathrm{H}), 6.94$ (s, 1H), 6.79 (d, J=7.3 Hz, 2H), 6.42 (s, 1H), 5.71 (s, $1 \mathrm{H}), 3.24(\mathrm{~m}, 1 \mathrm{H}), 3.02(\mathrm{~m}, 1 \mathrm{H}), 2.30(\mathrm{~s}, 3 \mathrm{H}), 2.30(\mathrm{~s}, 3 \mathrm{H}), 1.86(\mathrm{~m}, 2 \mathrm{H}), 1.10(\mathrm{t}, J=$ $7.6 \mathrm{~Hz}, 3 \mathrm{H}) .{ }^{13} \mathrm{C} \mathrm{NMR}\left(100 \mathrm{MHz}, \mathrm{CDCl}_{3}\right) \delta 141.7,138.1,135.9,135.1,131.8,129.9$, $129.5,128.9,127.8,125.4,125.8,129.7,117.5,116.7,98.0,58.2,20.6,17.7,16.5,13.2$. HRMS m/z calcd for $\mathrm{C}_{25} \mathrm{H}_{26} \mathrm{~N}_{2} \mathrm{NaOS}\left([\mathrm{M}+\mathrm{Na}]^{+}\right)$: 425.1658 , found 425.1694 .

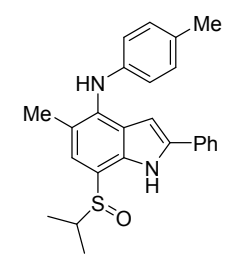

7-(isopropylsulfinyl)-5-methyl-2-phenyl- $N$-(p-tolyl)-1H-indol-4-amine 38: (eluent: petroleum ether/EtOAc 5:1), $22 \mathrm{mg}, 55 \%$; gray oil; ${ }^{1} \mathrm{H}$ NMR (400 $\left.\mathrm{MHz}, \mathrm{CDCl}_{3}\right) \delta$ $10.36(\mathrm{~s}, 1 \mathrm{H}), 7.59$ (d, $J=7.6 \mathrm{~Hz}, 2 \mathrm{H}), 7.39$ (t, $J=7.6 \mathrm{~Hz}, 2 \mathrm{H}), 7.30$ (t, $J=7.4 \mathrm{~Hz}, 1 \mathrm{H})$, 
$7.05(\mathrm{~d}, J=7.9 \mathrm{~Hz}, 2 \mathrm{H}), 6.91(\mathrm{~s}, 1 \mathrm{H}), 6.78(\mathrm{~d}, J=7.9 \mathrm{~Hz}, 2 \mathrm{H}), 6.41(\mathrm{~d}, J=2.4 \mathrm{~Hz}, 1 \mathrm{H})$, $5.70(\mathrm{~s}, 1 \mathrm{H}), 3.2(\mathrm{p}, J=6.9 \mathrm{~Hz}, 1 \mathrm{H}), 2.30(\mathrm{~s}, 6 \mathrm{H}), 1.38(\mathrm{~d}, J=6.9 \mathrm{~Hz}, 3 \mathrm{H}), 1.26(\mathrm{~d}, J=$ $6.9 \mathrm{~Hz}, 3 \mathrm{H}) .{ }^{13} \mathrm{C} \mathrm{NMR}\left(100 \mathrm{MHz}, \mathrm{CDCl}_{3}\right) \delta 141.7,138.0,135.8,135.8,131.8,129.9$, $129.5,128.8,127.8,125.4,125.2,122.9,119.5,117.4,114.7,97.8,55.0,20.6,17.7$, 16.3, 15.3. HRMS m/z calcd for $\mathrm{C}_{25} \mathrm{H}_{27} \mathrm{~N}_{2} \mathrm{OS}\left([\mathrm{M}+\mathrm{H}]^{+}\right)$: 403.1839 , found 403.1832 .

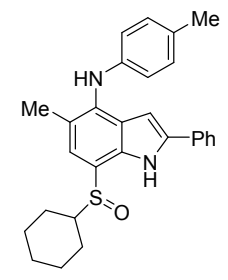

7-(cyclohexylsulfinyl)-5-methyl-2-phenyl- $N$-(p-tolyl)-1H-indol-4-amine 39: (eluent: petroleum ether/EtOAc 5:1), $23 \mathrm{mg}, 51 \%$; gray oil; ${ }^{1} \mathrm{H}$ NMR $\left(400 \mathrm{MHz}, \mathrm{CDCl}_{3}\right) \delta$ 10.34 (s, 1H), 7.60 (d, $J=7.7 \mathrm{~Hz}, 2 \mathrm{H}), 7.40$ (t, $J=7.6 \mathrm{~Hz}, 2 \mathrm{H}), 7.30$ (t, $J=7.4 \mathrm{~Hz}, 1 \mathrm{H})$, $7.05(\mathrm{~d}, J=7.9 \mathrm{~Hz}, 2 \mathrm{H}), 6.90$ (s, 1H), 6.79 (d, $J=7.9 \mathrm{~Hz}, 2 \mathrm{H}), 6.41(\mathrm{~d}, J=2.4 \mathrm{~Hz}, 1 \mathrm{H})$, 5.71(s, 1H), $2.97(\mathrm{~m}, 1 \mathrm{H}), 2.31(\mathrm{~s}, 3 \mathrm{H}), 2.30(\mathrm{~s}, 3 \mathrm{H}), 2.11(\mathrm{~m}, 2 \mathrm{H}), 1.92(\mathrm{~m}, 3 \mathrm{H}), 1.67$ (m, 5H). ${ }^{13} \mathrm{C} \mathrm{NMR}\left(100 \mathrm{MHz}, \mathrm{CDCl}_{3}\right) \delta 141.8,138.0,135.8,135.8,131.8,129.9,129.5$, $128.8,127.8,125.4,125.1,122.8,119.5,117.5,114.7,97.8,63.1,26.4,25.5,25.3,25.2$, 20.6, 17.8. HRMS m/z calcd for $\mathrm{C}_{28} \mathrm{H}_{31} \mathrm{~N}_{2} \mathrm{OS}\left([\mathrm{M}+\mathrm{H}]^{+}\right)$: 443.2152, found 443.2080.

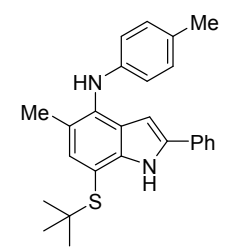

7-(tert-butylthio)-5-methyl-2-phenyl- $N$-(p-tolyl)-1H-indol-4-amine $\quad 41$ : (eluent: petroleum ether/EtOAc 5:1), $25 \mathrm{mg}, 62 \%$; yellow solid; m.p. $133-134{ }^{\circ} \mathrm{C}$; ${ }^{1} \mathrm{H}$ NMR $\left(400 \mathrm{MHz}, \mathrm{CDCl}_{3}\right) \delta 8.70(\mathrm{~s}, 1 \mathrm{H}), 7.58(\mathrm{~d}, J=7.2 \mathrm{~Hz}, 2 \mathrm{H}), 7.40(\mathrm{t}, J=7.7 \mathrm{~Hz}, 2 \mathrm{H})$, $7.28(\mathrm{t}, J=7.4 \mathrm{~Hz}, 1 \mathrm{H}), 7.21(\mathrm{~s}, 1 \mathrm{H}), 7.02(\mathrm{~d}, J=8.2 \mathrm{~Hz}, 2 \mathrm{H}), 6.74(\mathrm{~d}, J=8.4 \mathrm{~Hz}, 2 \mathrm{H})$, $6.49(\mathrm{~d}, J=2.4 \mathrm{~Hz}, 1 \mathrm{H}), 5.60(\mathrm{~s}, 1 \mathrm{H}), 2.31(\mathrm{~s}, 3 \mathrm{H}), 2.28(\mathrm{~s}, 3 \mathrm{H}), 1.37(\mathrm{~s}, 9 \mathrm{H}) . ;{ }^{13} \mathrm{C} \mathrm{NMR}$ $\left(100 \mathrm{MHz}, \mathrm{CDCl}_{3}\right) \delta 142.5,140.6,136.5,135.2,133.8,132.2,129.5,128.5,127.6$, 125.1, 123.8, 121.6, 116.4, 108.4, 99.3, 47.5, 31.3, 20.6, 17.7. HRMS m/z calcd for $\mathrm{C}_{26} \mathrm{H}_{29} \mathrm{~N}_{2} \mathrm{~S}\left([\mathrm{M}+\mathrm{H}]^{+}\right)$: 401.2046, found 401.2038.

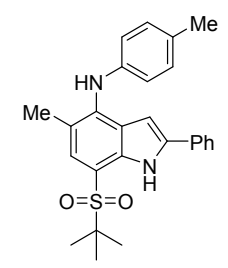


7-(tert-butylsulfonyl)-5-methyl-2-phenyl- $N$-(p-tolyl)-1H-indol-4-amine 42: (eluent: petroleum ether/EtOAc 5:1), $40 \mathrm{mg}, 93 \%$; yellow solid; m.p. 163-164 ${ }^{\circ} \mathrm{C} ;{ }^{1} \mathrm{H}$ NMR $\left(400 \mathrm{MHz}, \mathrm{CDCl}_{3}\right) \delta 9.69(\mathrm{~s}, 1 \mathrm{H}), 7.51(\mathrm{~d}, J=7.3 \mathrm{~Hz}, 2 \mathrm{H}), 7.41(\mathrm{~s}, 1 \mathrm{H}), 7.38(\mathrm{t}, J=7.6$ $\mathrm{Hz}, 2 \mathrm{H}), 7.28$ (t, $J=7.3 \mathrm{~Hz}, 1 \mathrm{H}), 7.09$ (d, $J=8.0 \mathrm{~Hz}, 2 \mathrm{H}), 6.91(\mathrm{~d}, J=8.3 \mathrm{~Hz}, 2 \mathrm{H})$, $6.25(\mathrm{~d}, J=2.4 \mathrm{~Hz}, 1 \mathrm{H}), 5.91(\mathrm{~s}, 1 \mathrm{H}), 2.34(\mathrm{~s}, 6 \mathrm{H}), 1.41(\mathrm{~s}, 9 \mathrm{H}) ;{ }^{13} \mathrm{C} \mathrm{NMR}(100 \mathrm{MHz}$, $\left.\mathrm{CDCl}_{3}\right) \delta 140.2,139.6,137.3,136.0,131.7,131.5,129.5,128.9,128.0,125.2,121.7$, 119.9, 117.2, 108.3, 99.0, 61.3, 23.5, 20.8, 17.8. HRMS m/z calcd for $\mathrm{C}_{26} \mathrm{H}_{29} \mathrm{~N}_{2} \mathrm{O}_{2} \mathrm{~S}$ $\left([\mathrm{M}+\mathrm{H}]^{+}\right):$433.1944, found 433.1922 .

\section{X-ray diffraction structure of compound 2}

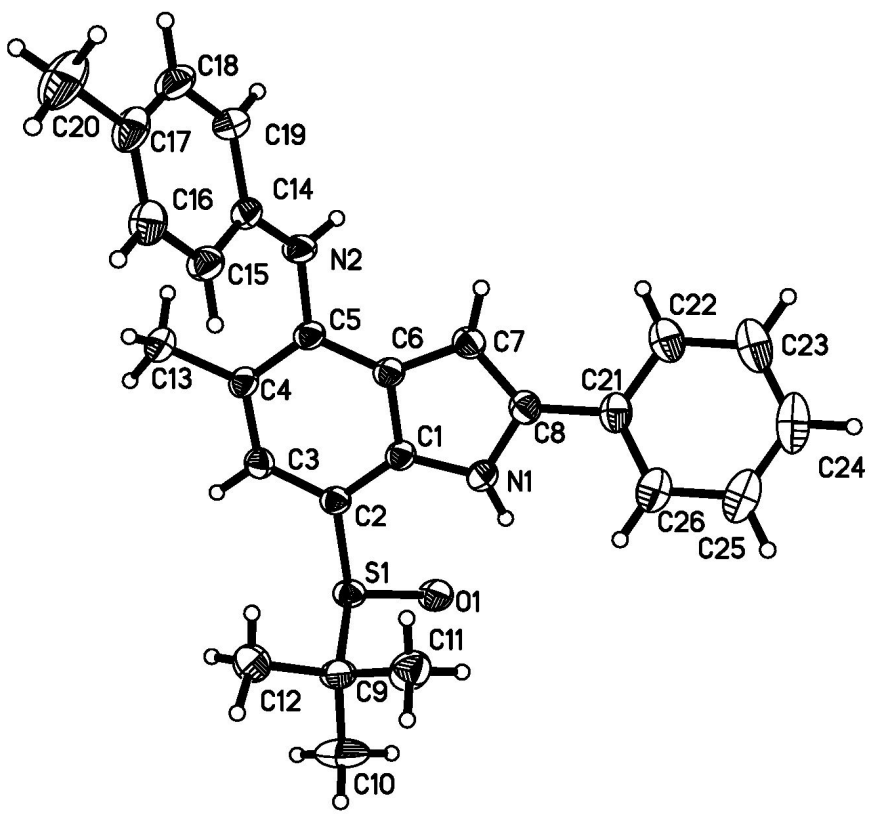




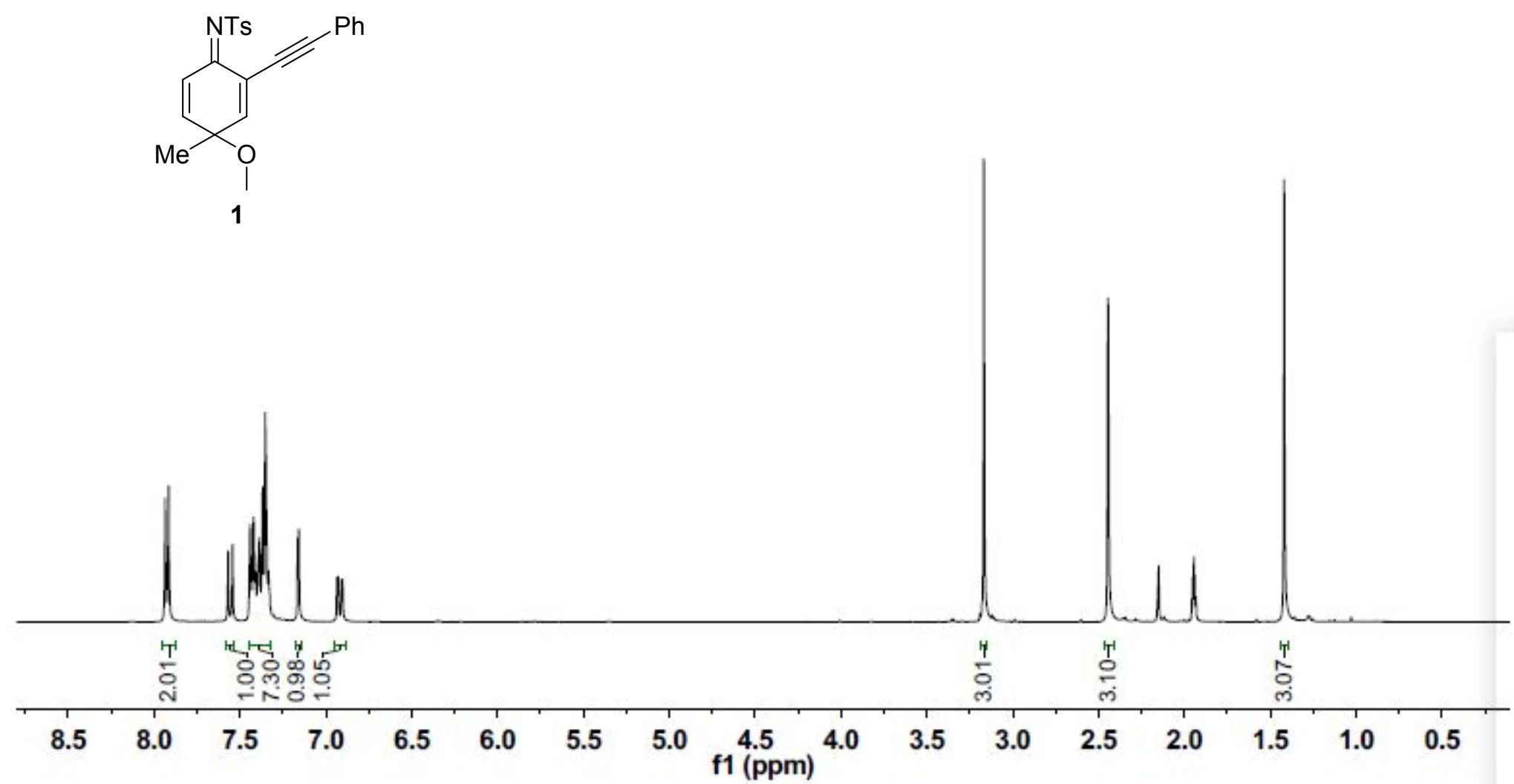




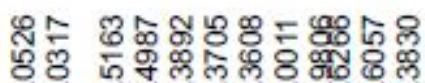

舟

$\frac{\infty}{5}$

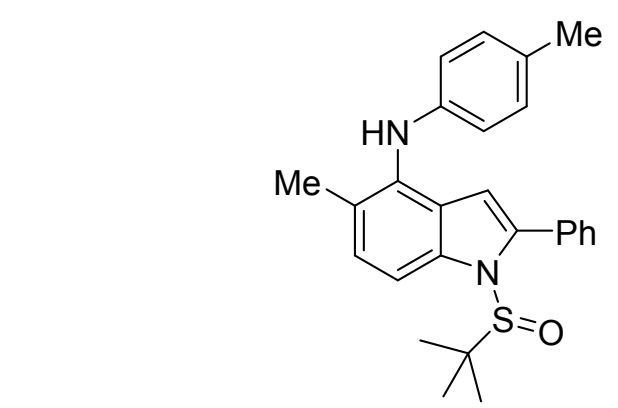

int-2B

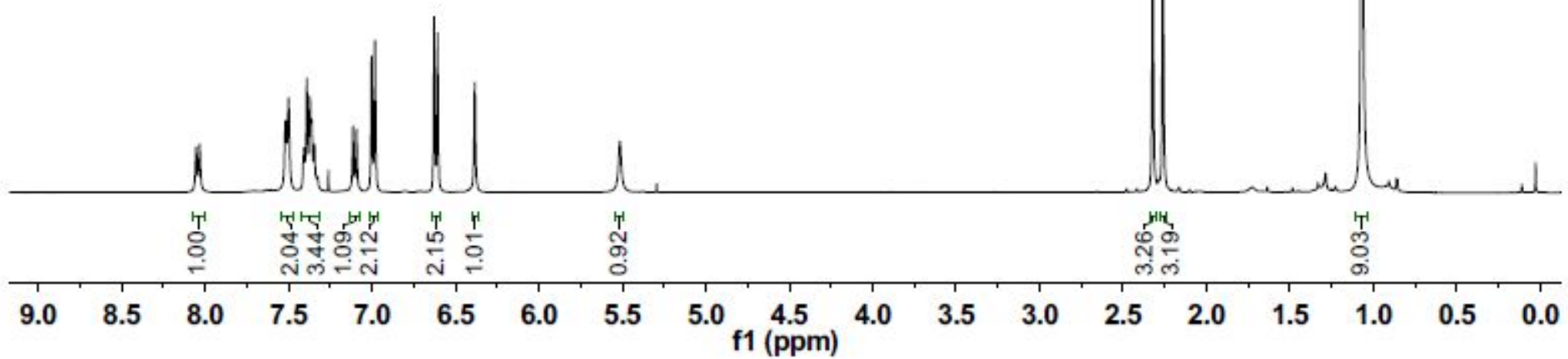




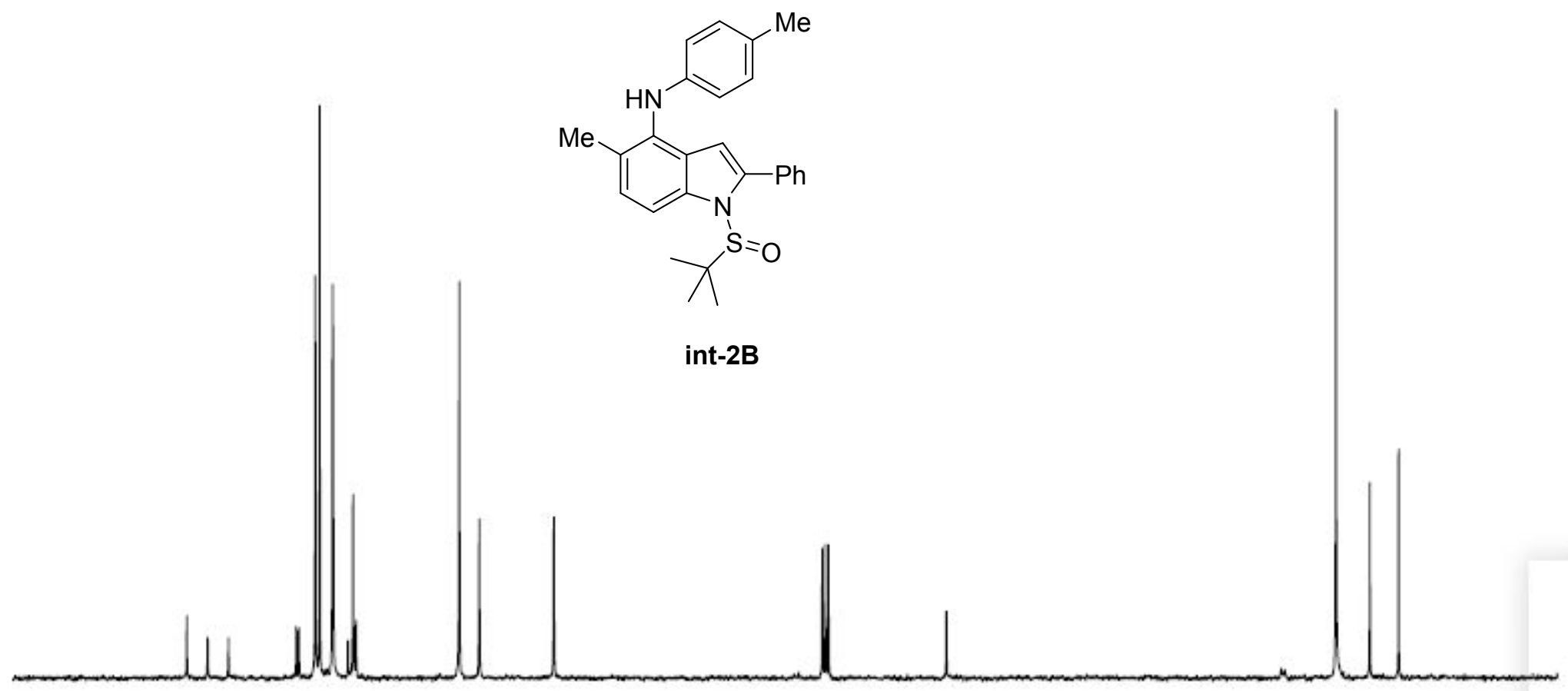




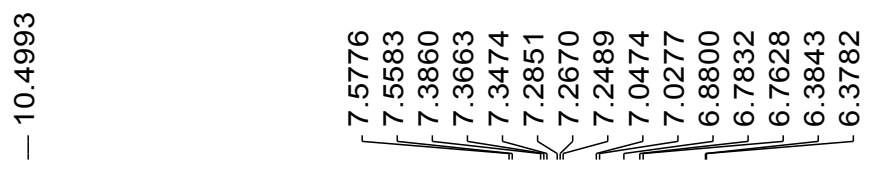

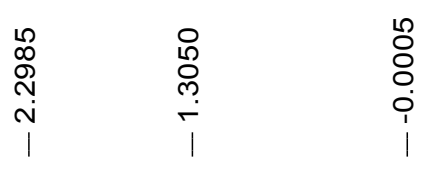

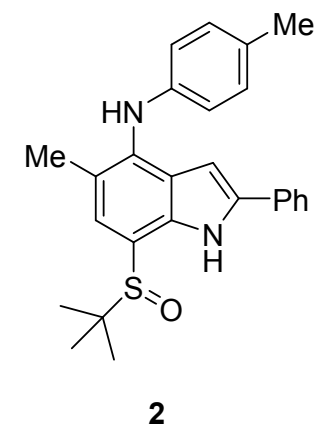

Ihill 年

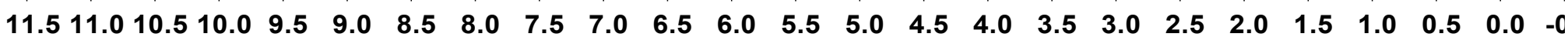
f1 (ppm) 


\begin{tabular}{|c|c|c|c|}
\hline 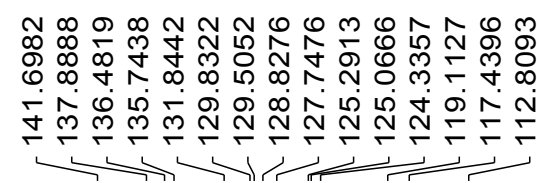 & $\begin{array}{l}\bar{g} \\
\stackrel{0}{0} \\
\hat{\sigma}\end{array}$ & 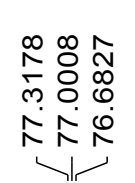 & 站 \\
\hline
\end{tabular}
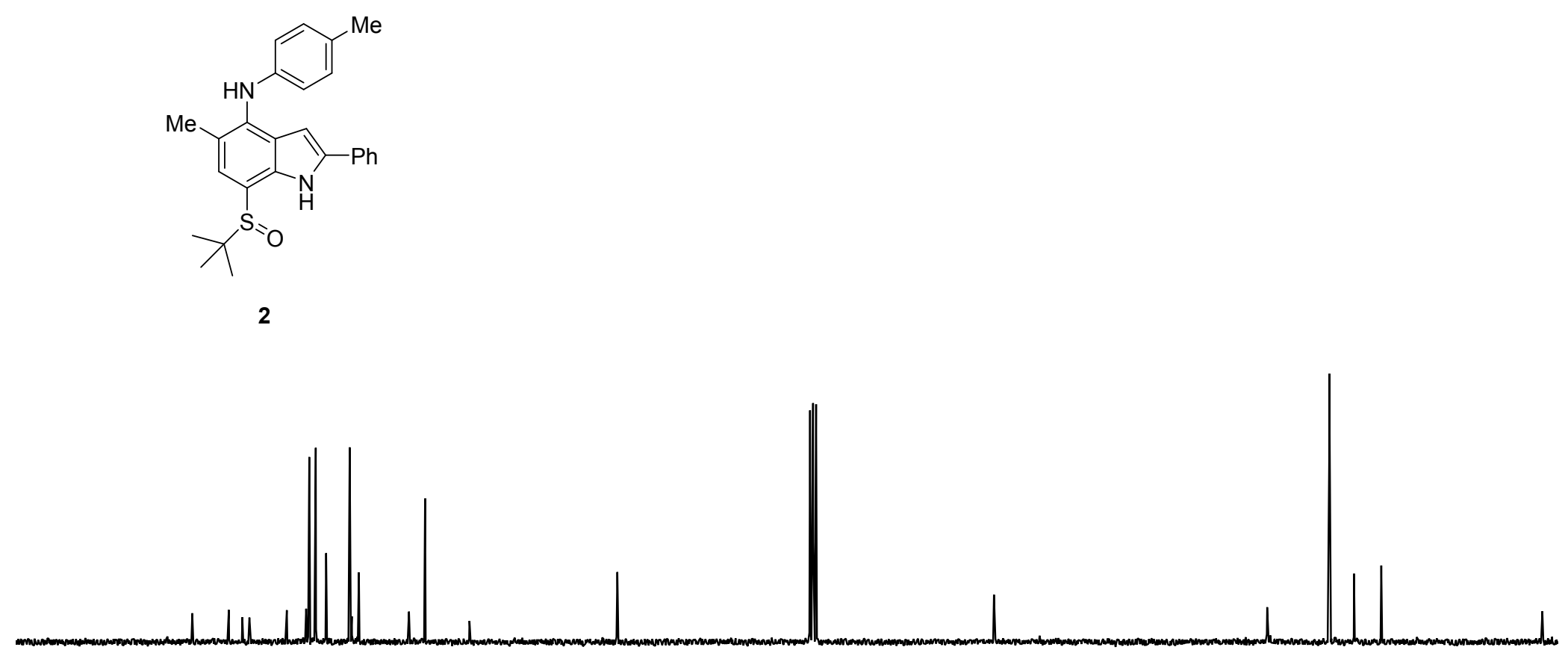

60

$150 \quad 140 \quad 130$

120

$110 \quad 100$

$90 \quad 80$

$\begin{array}{llllllll}70 & 60 & 50 & 40 & 30 & 20 & 10 & 0\end{array}$




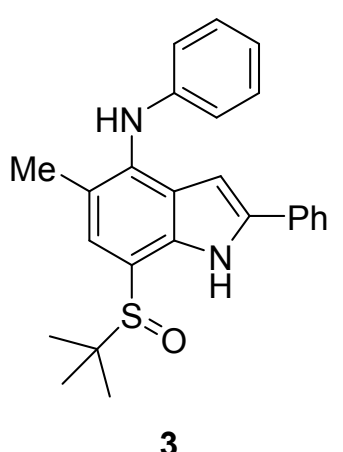

3

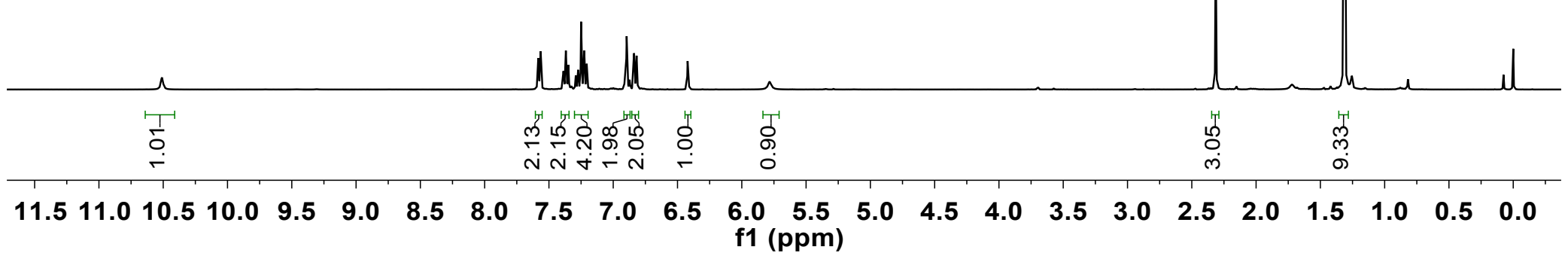



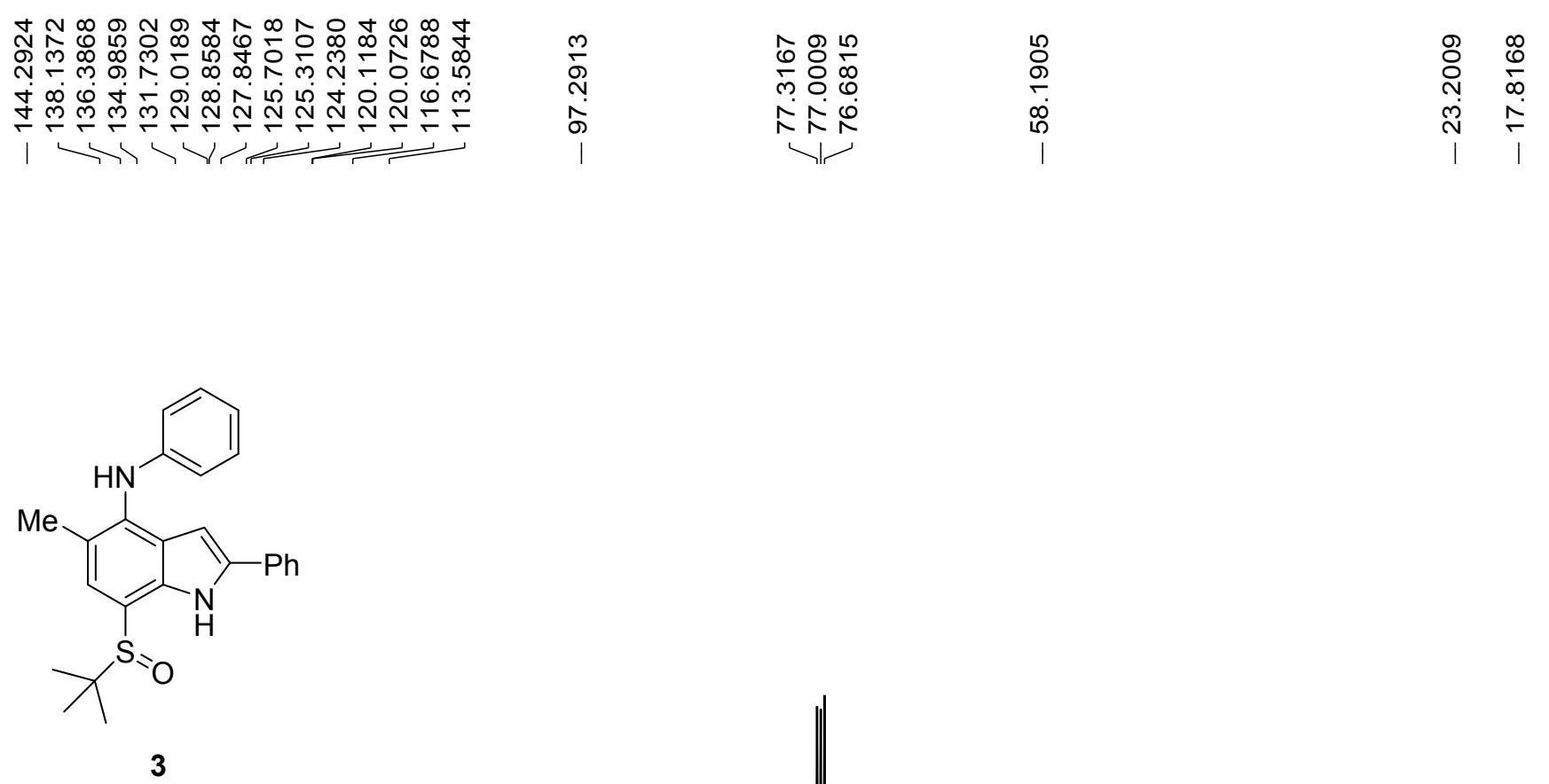

160
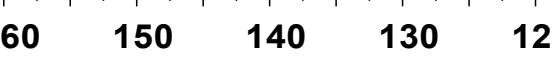

$110 \quad 100$

$90 \quad \begin{array}{r}80 \\ \text { f1 }\end{array}$

70

60

50

40

30

2010 


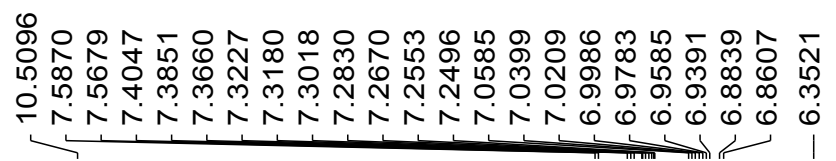

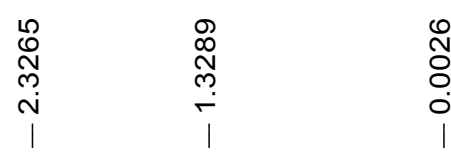
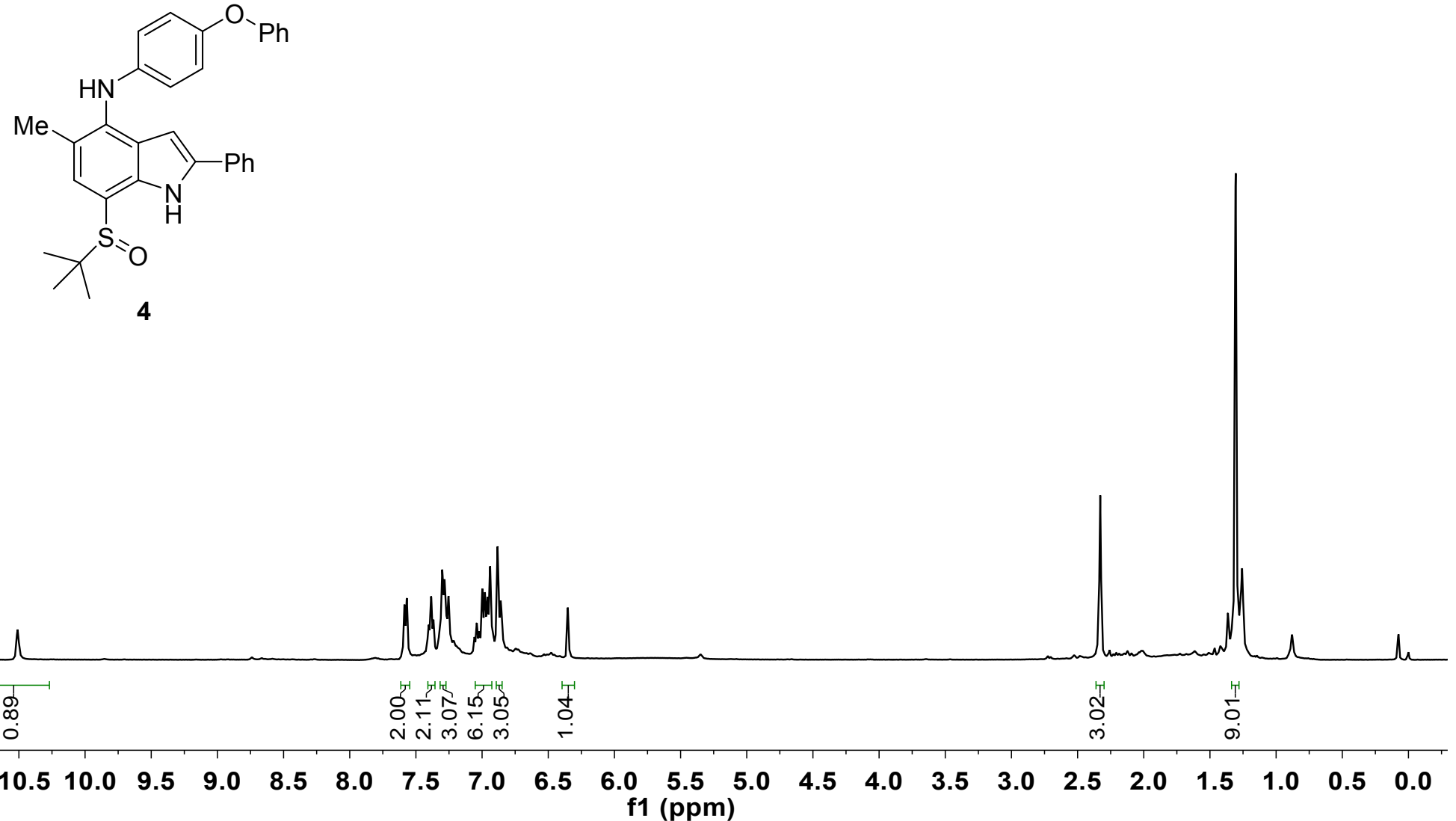


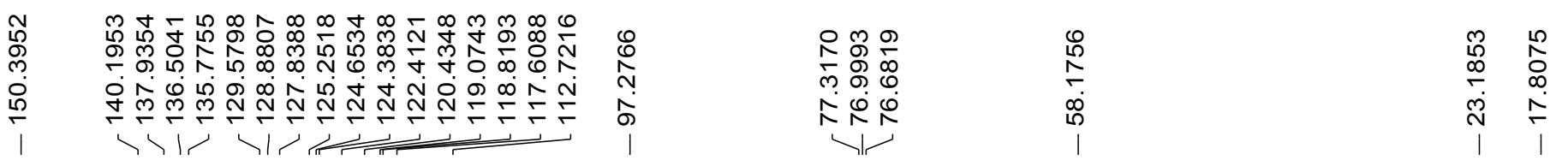<smiles>Cc1cc(S(=O)(=O)C(C)(C)C)c2[nH]c(-c3ccccc3)cc2c1Nc1ccc(Oc2ccccc2)cc1</smiles>

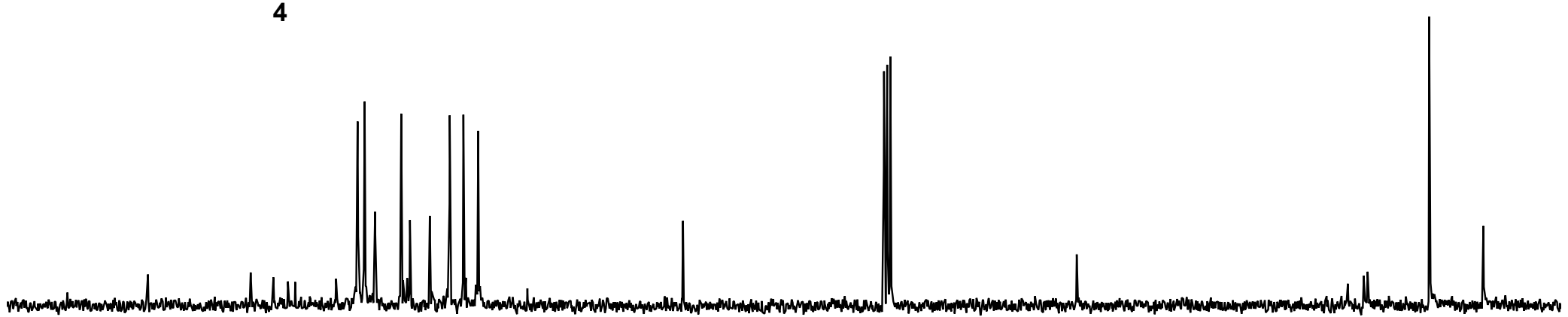




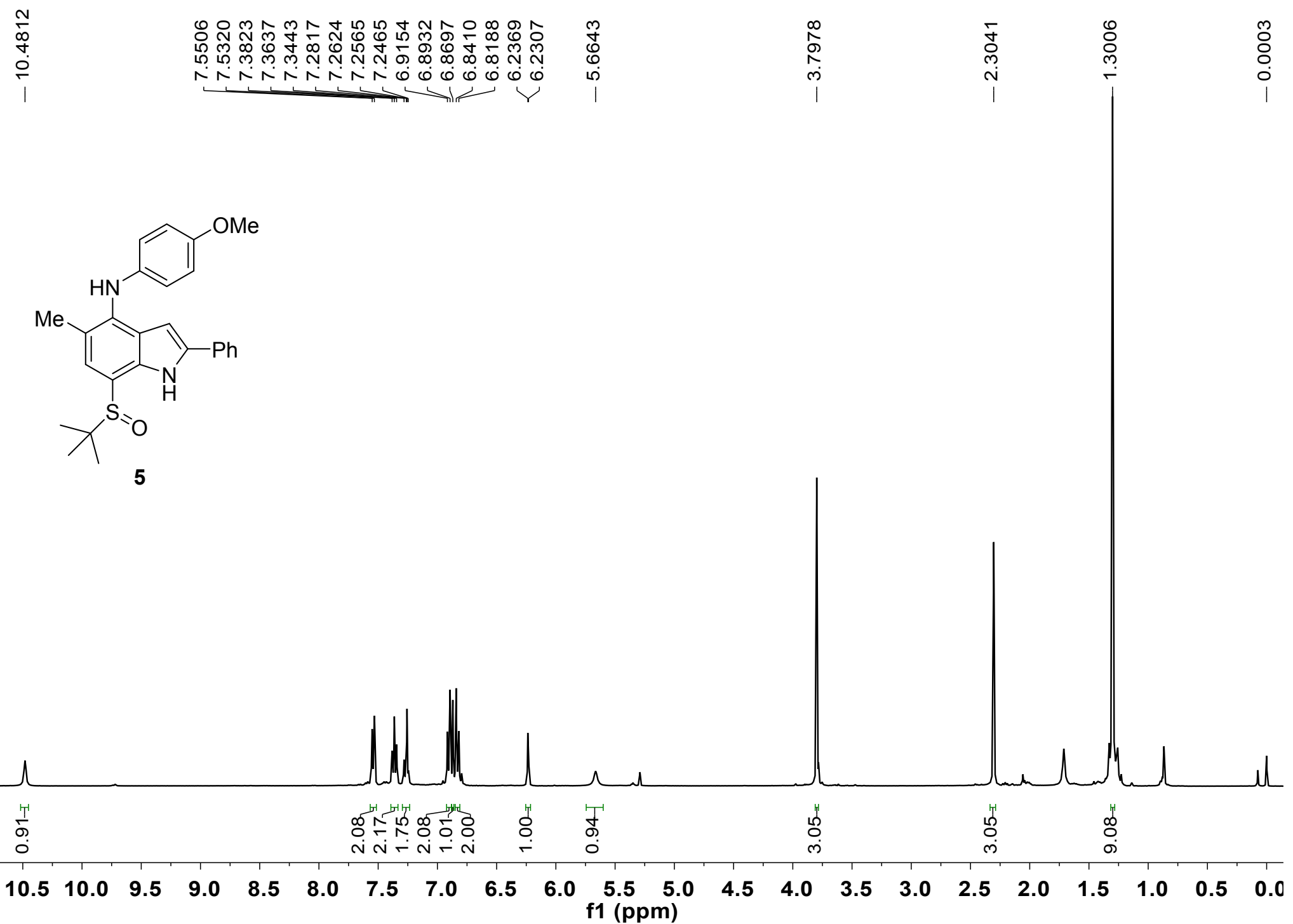




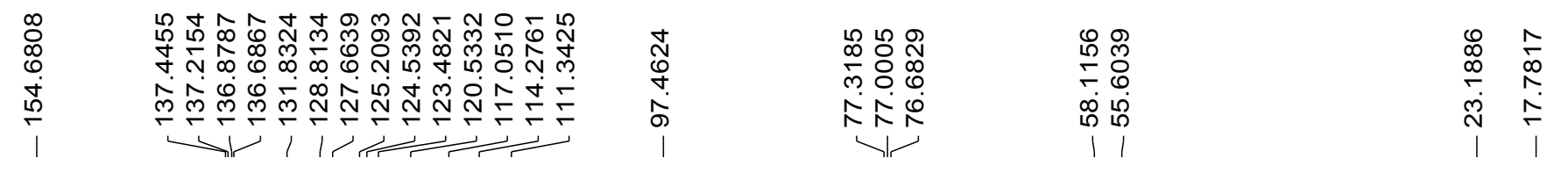
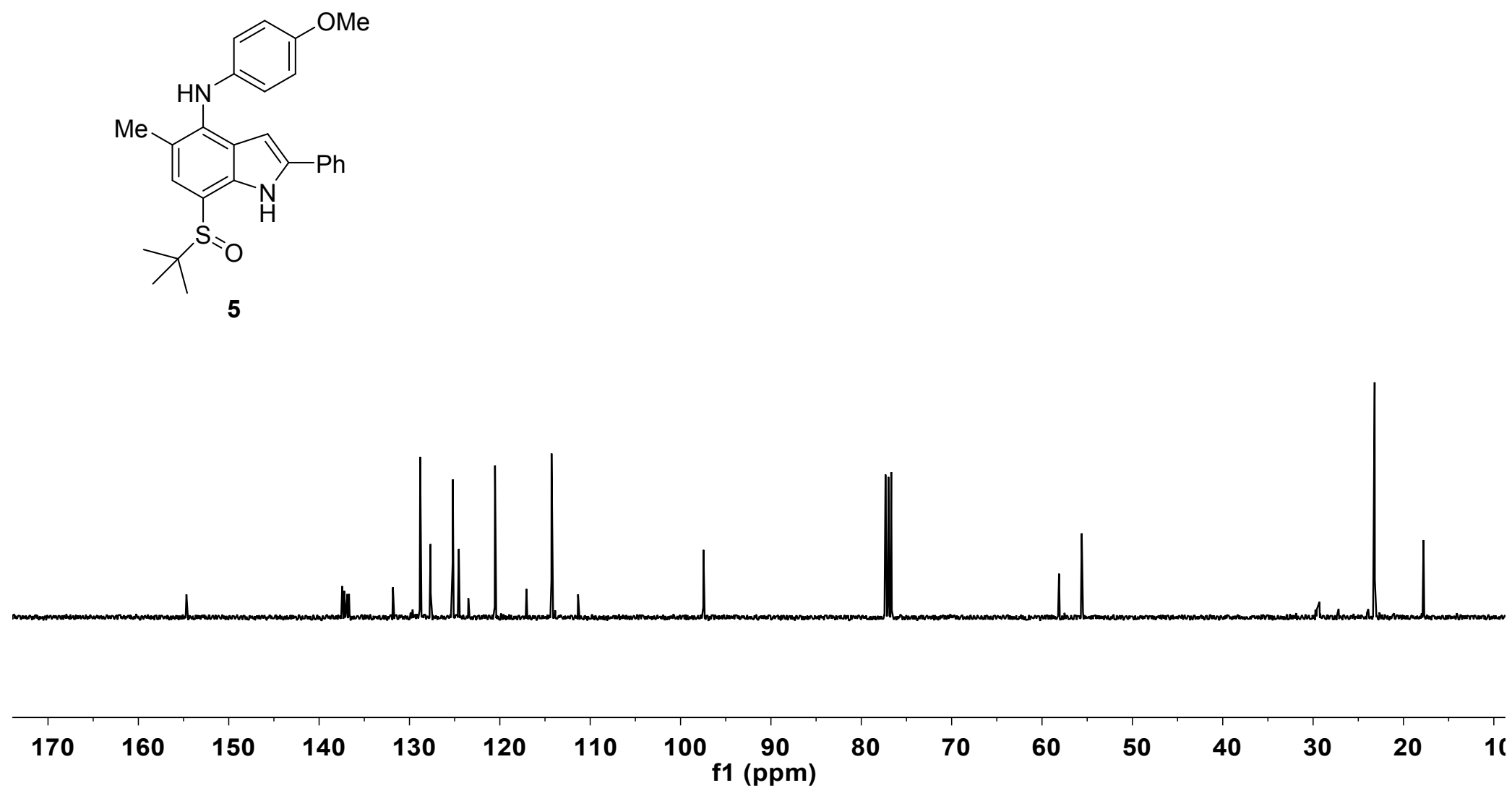

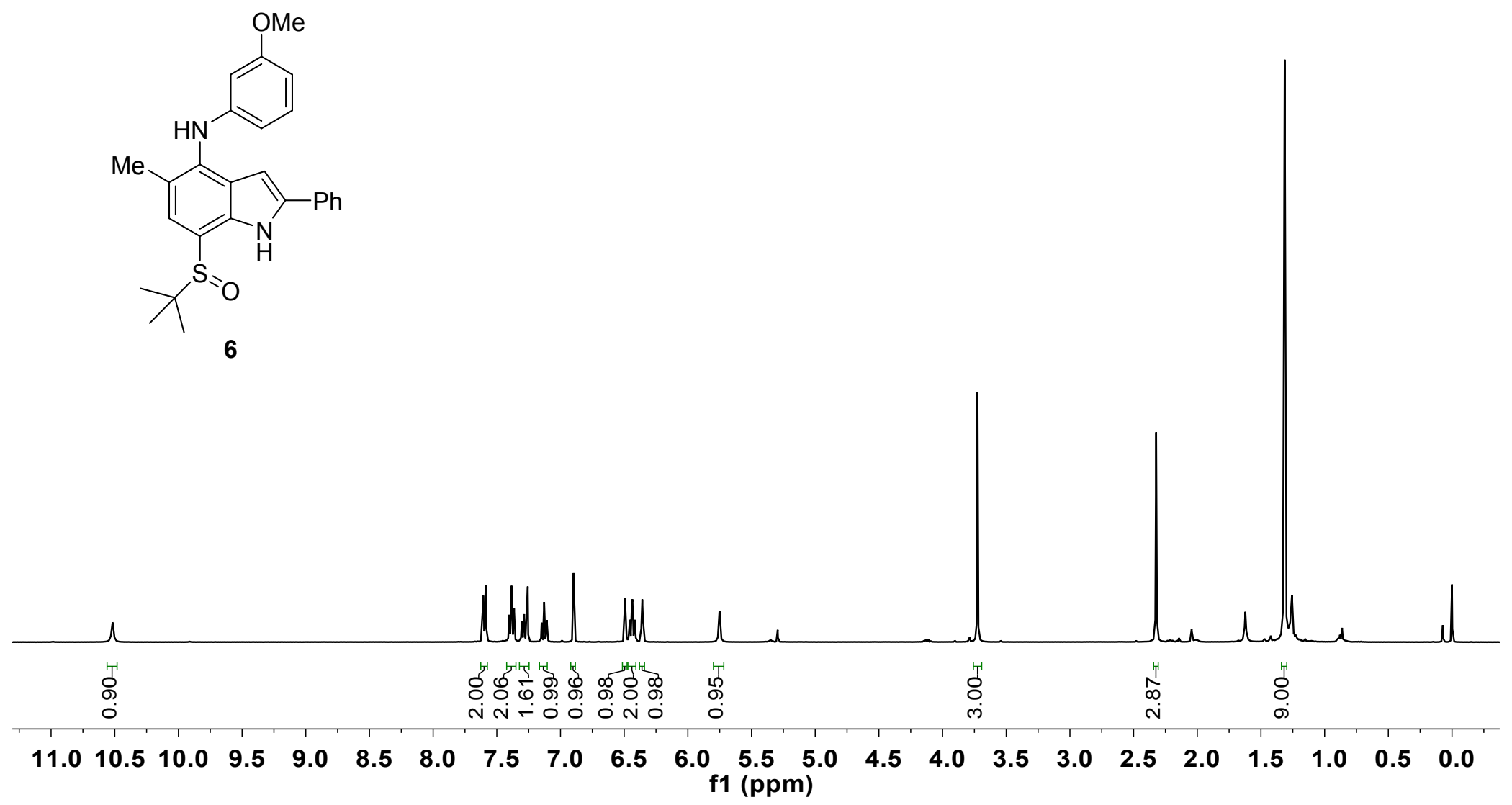


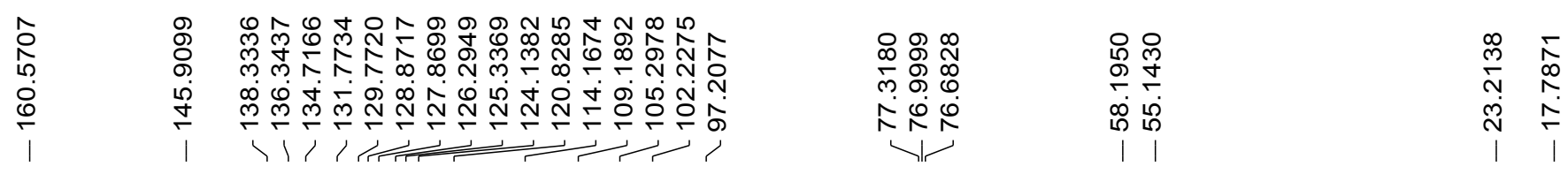<smiles>COc1cccc(Nc2c(C)cc(S(=O)C(C)(C)C)c3[nH]c(-c4ccccc4)cc23)c1</smiles>
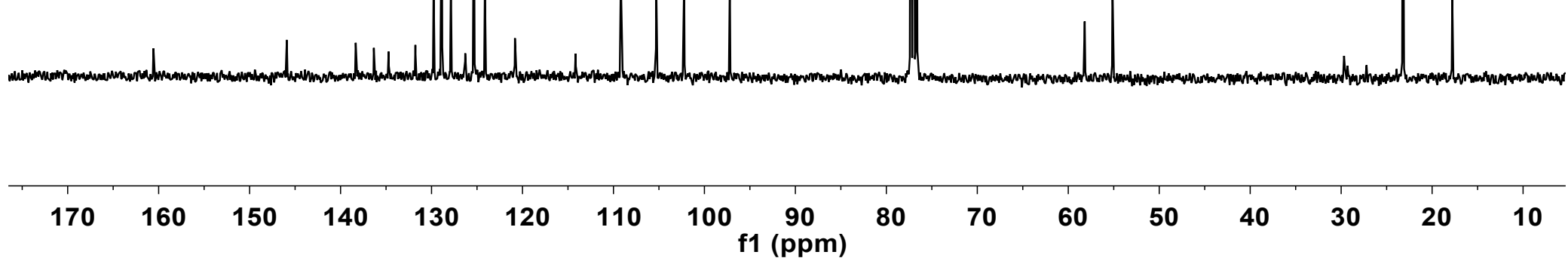

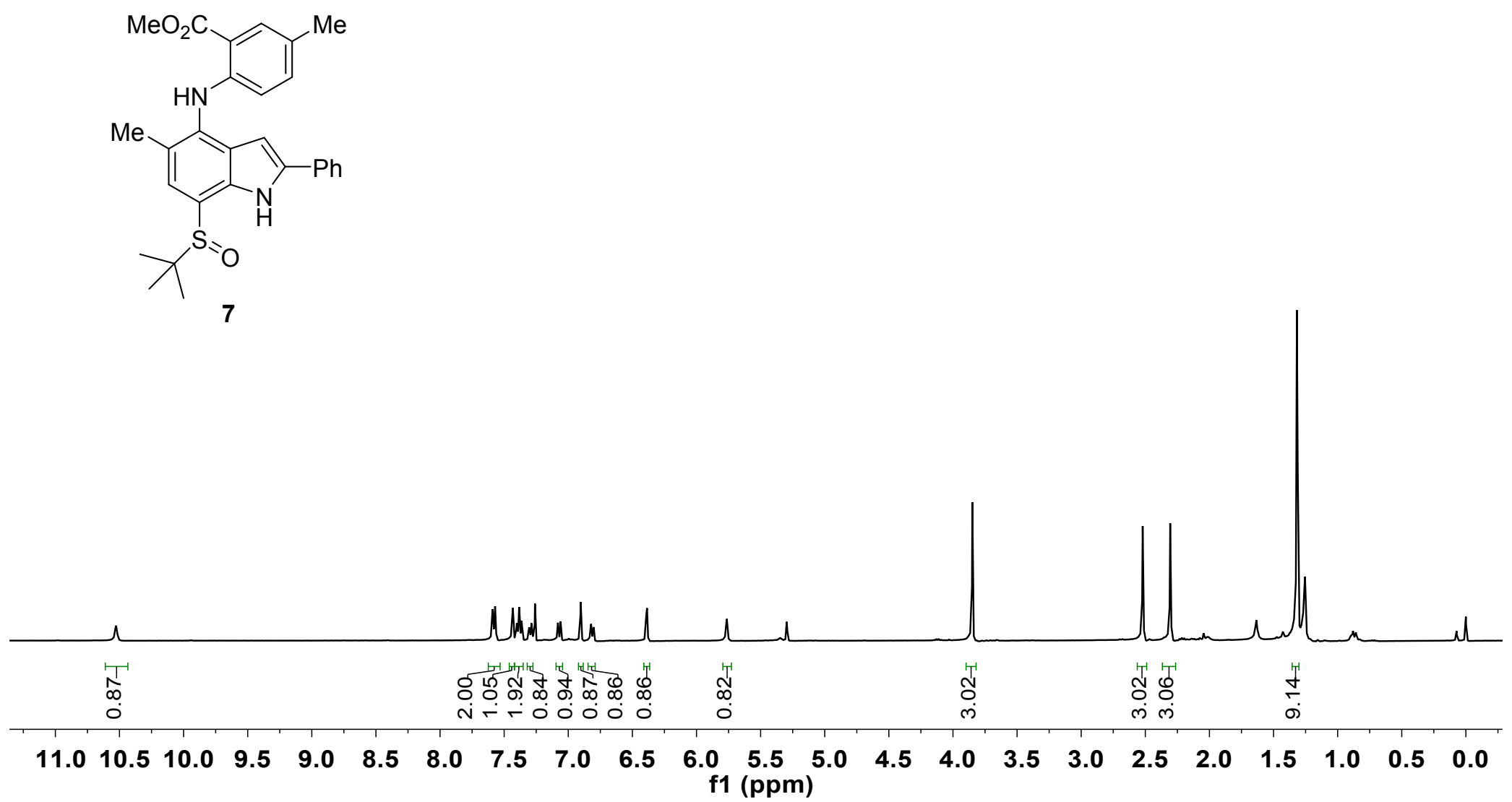


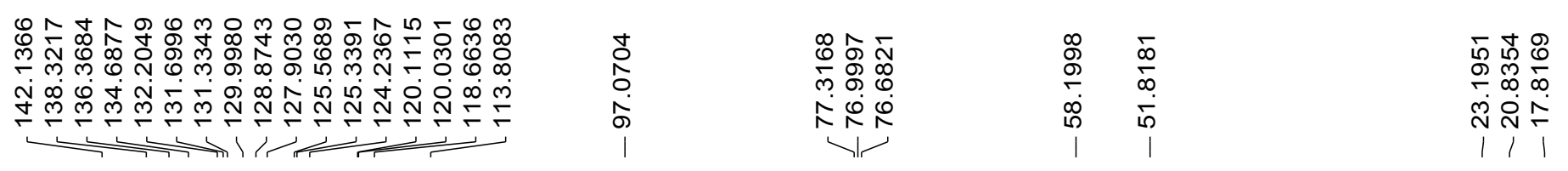<smiles>CC(=O)c1cc(C)ccc1Nc1c(C)cc(S(=O)C(C)(C)C)c2[nH]c(-c3ccccc3)cc12</smiles>

$\begin{array}{cccccccc}80 & 70 & 60 & 50 & 40 & 30 & 20 & 10\end{array}$



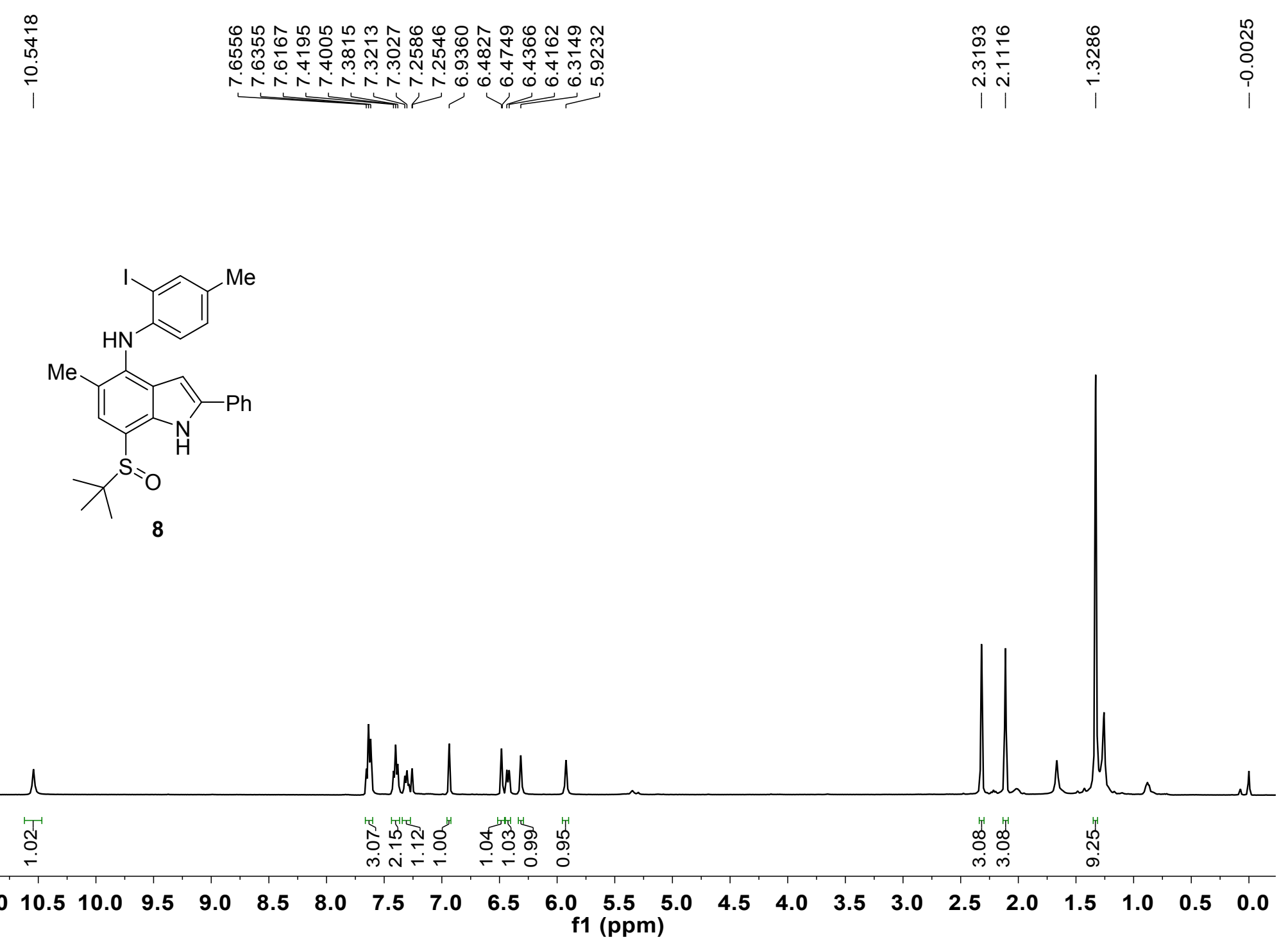

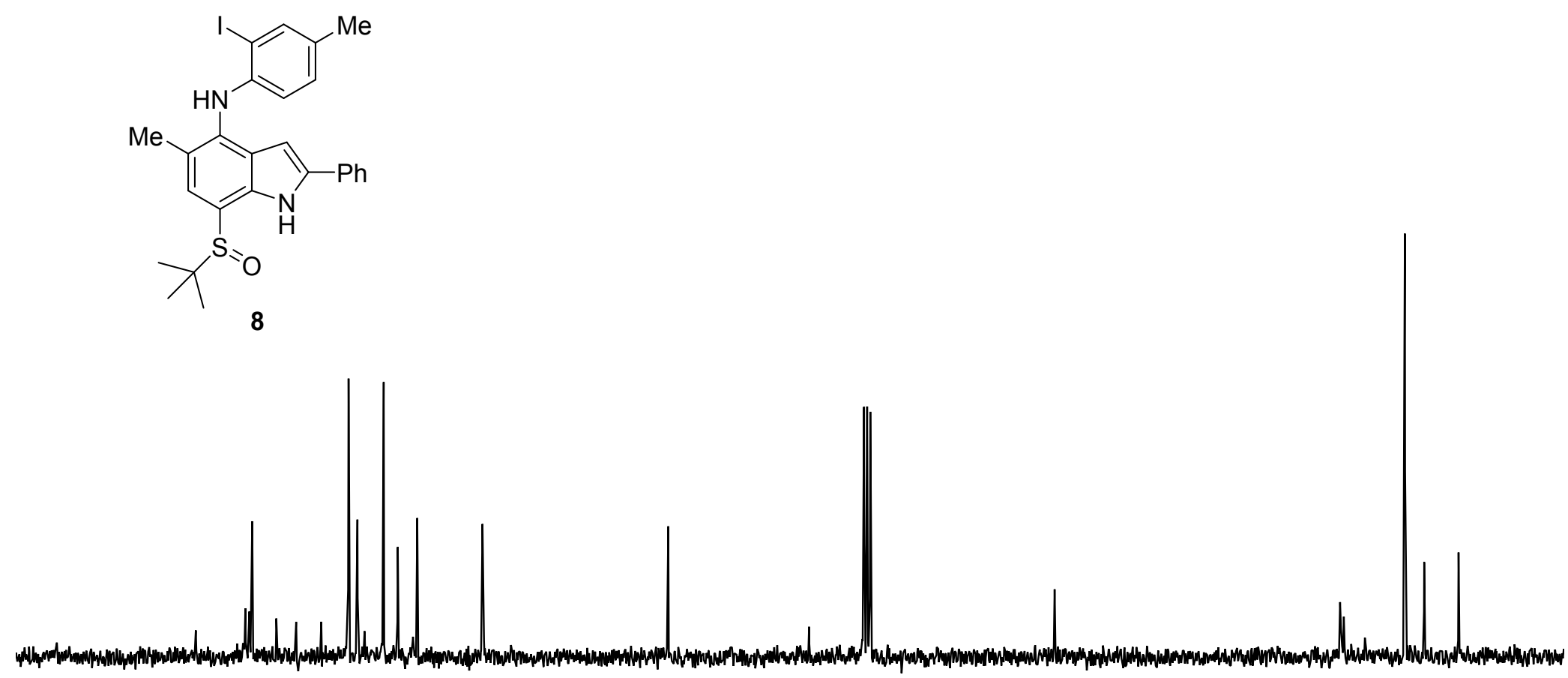
90
$\begin{array}{cr}90 & 80 \\ \text { f1 } & (p p m)\end{array}$

$\begin{array}{lllllll}70 & 60 & 50 & 40 & 30 & 20 & 10\end{array}$



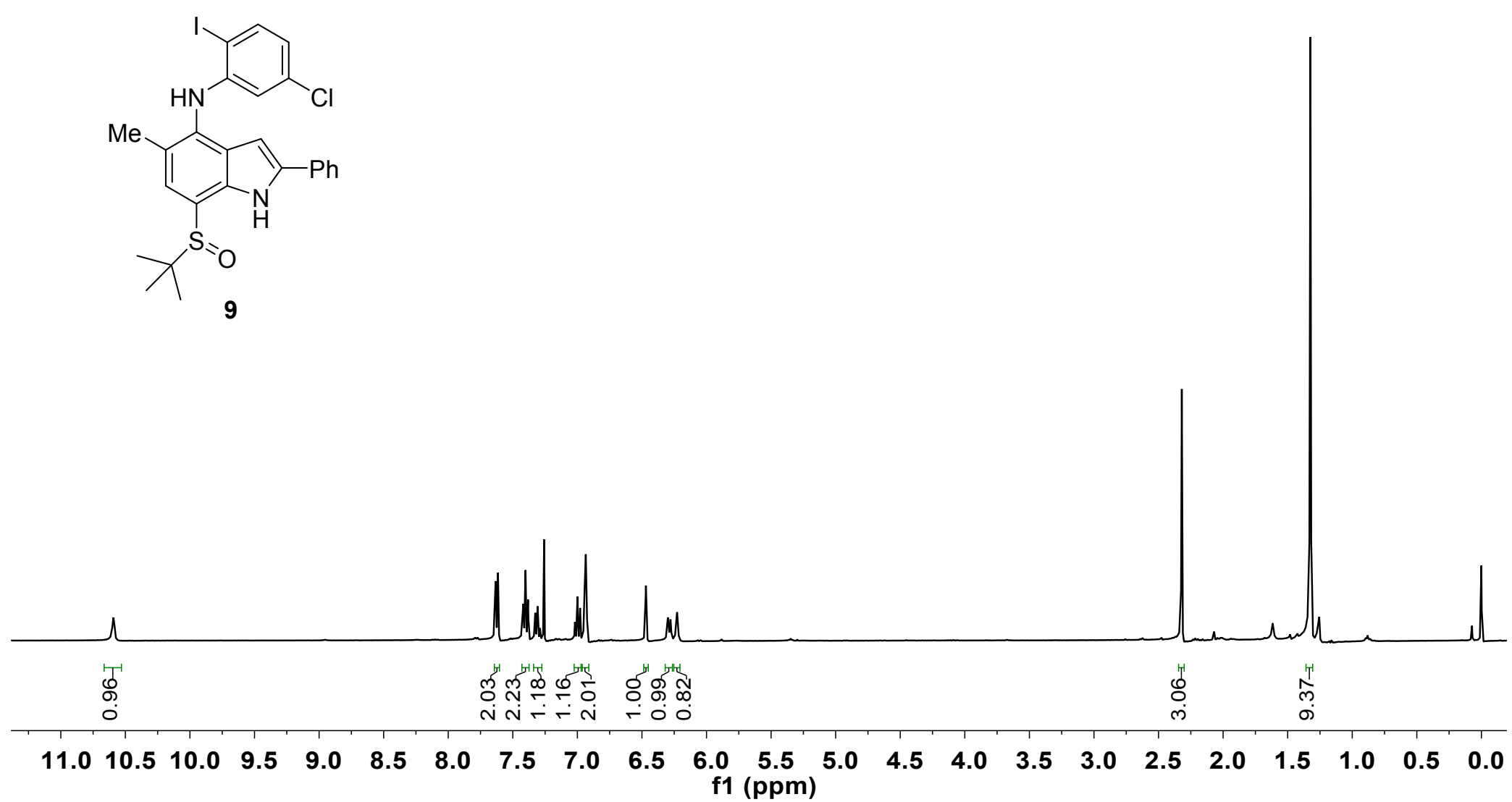


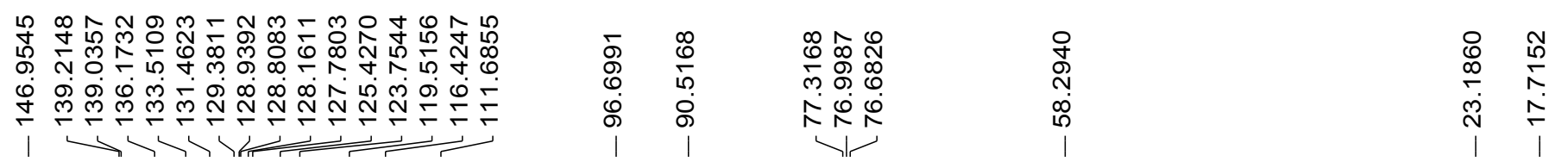<smiles>Cc1cc(S(=O)C(C)(C)C)c2[nH]c(-c3ccccc3)cc2c1Nc1cc(Cl)ccc1I</smiles>

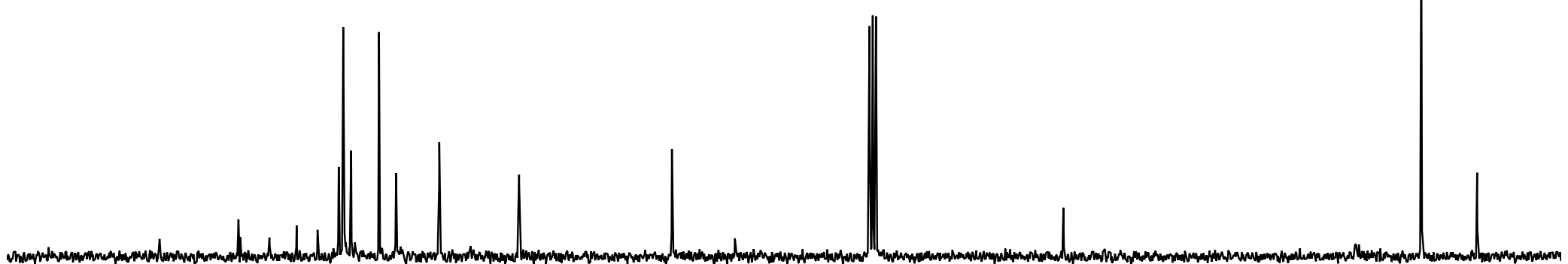



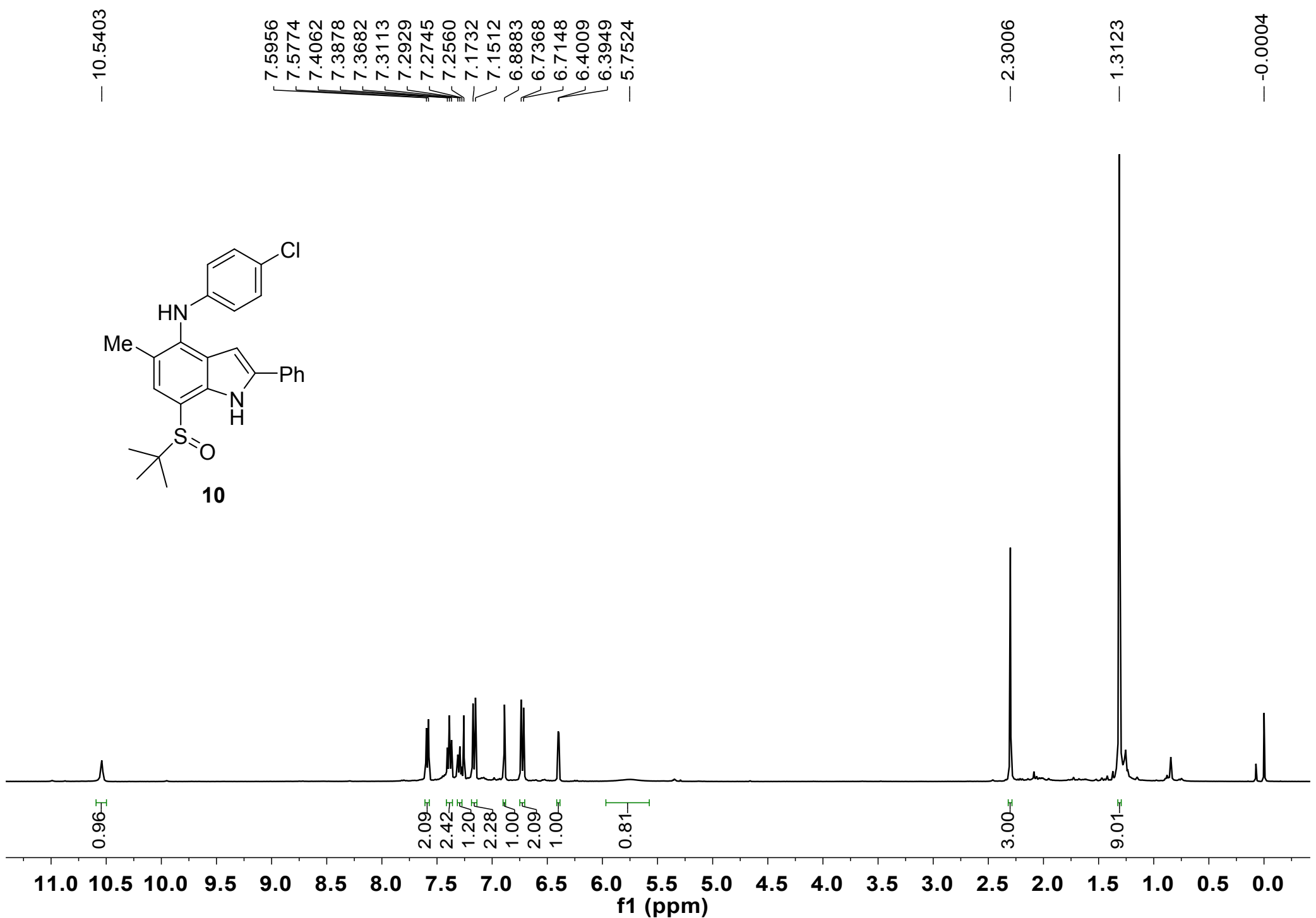


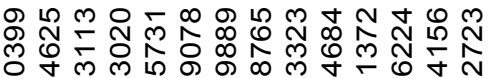

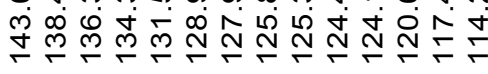

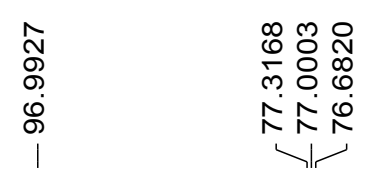

$\stackrel{\substack{N \\ \\ \infty \\ 1 \\ 1}}{1}$

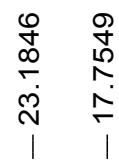

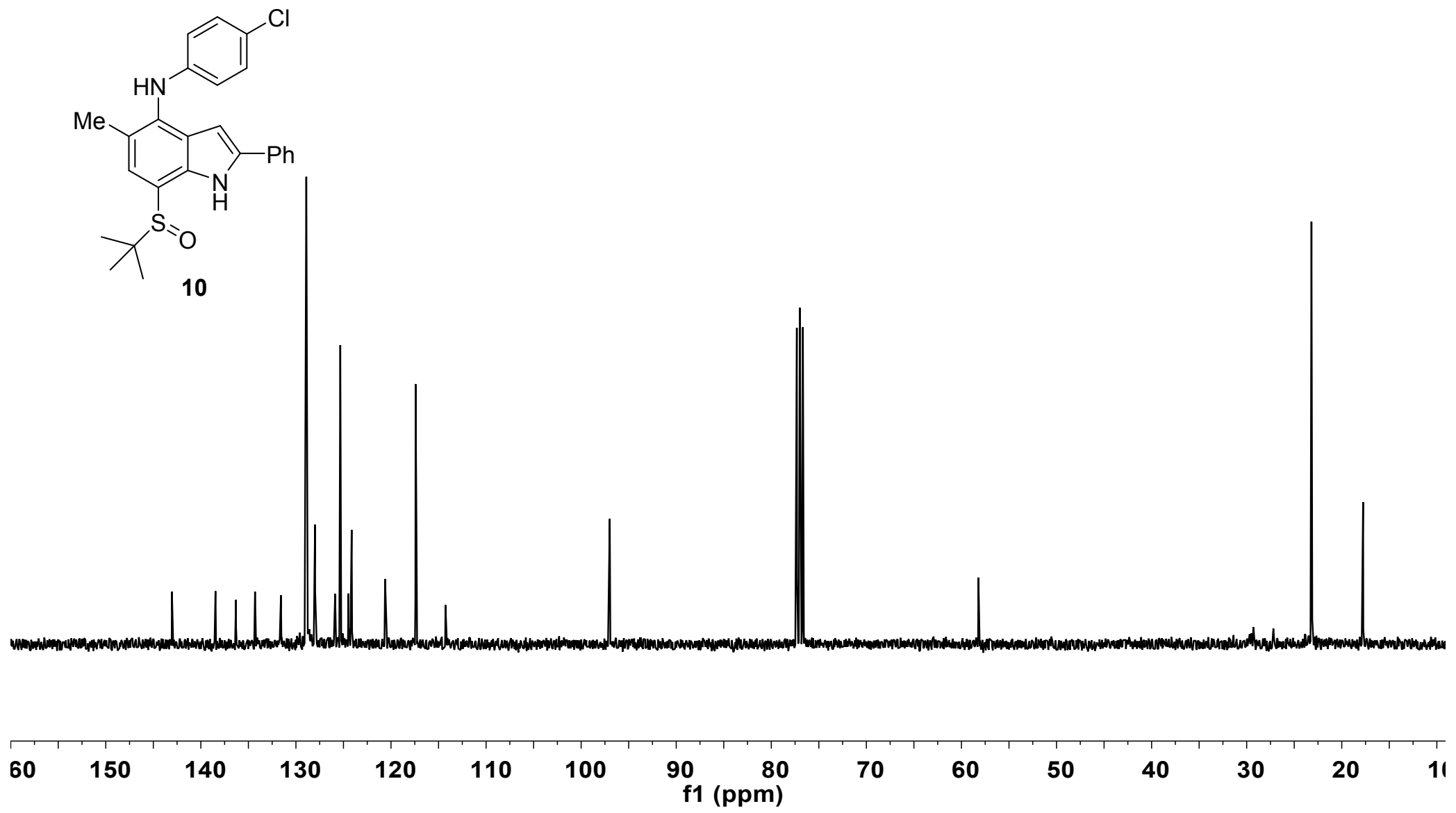



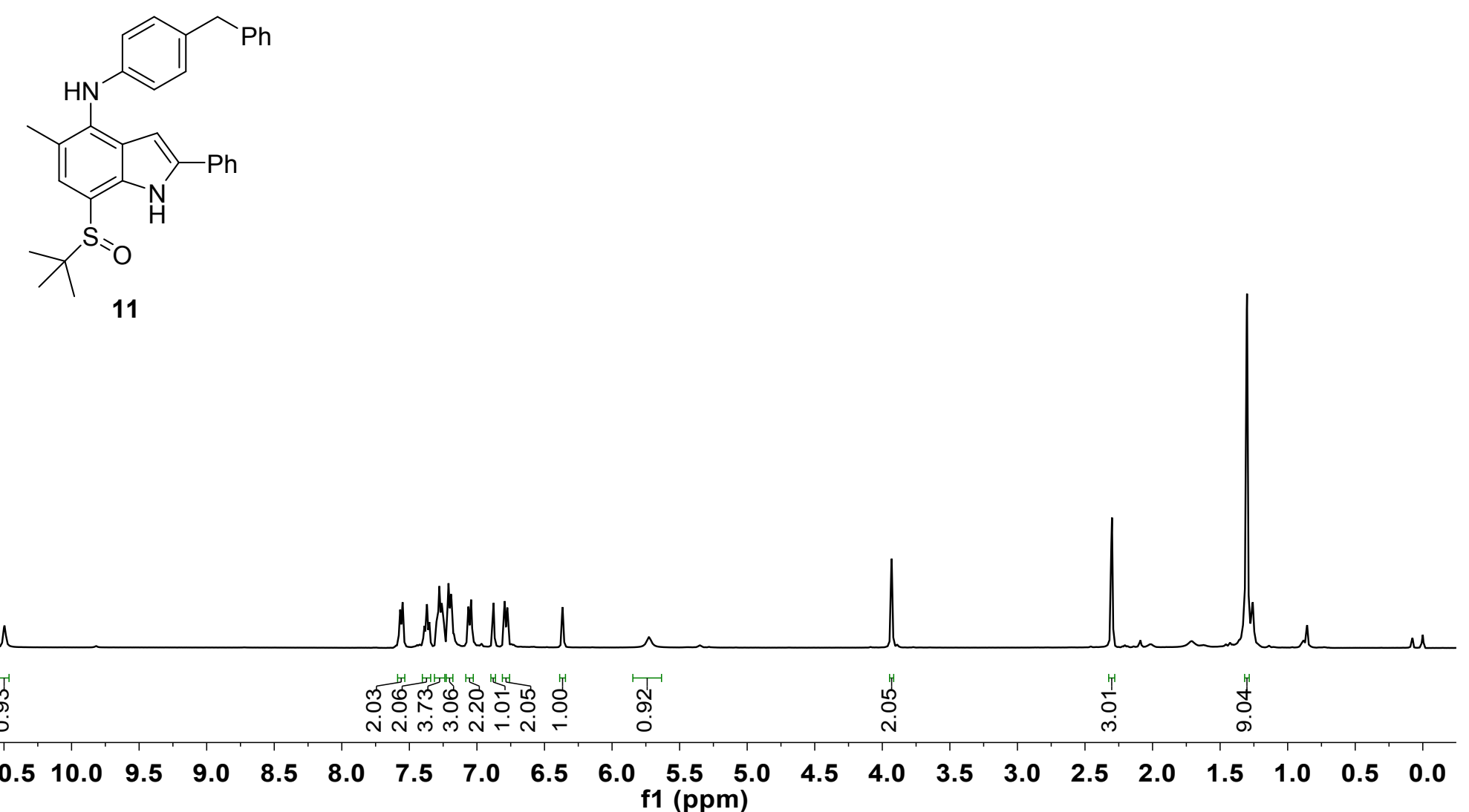


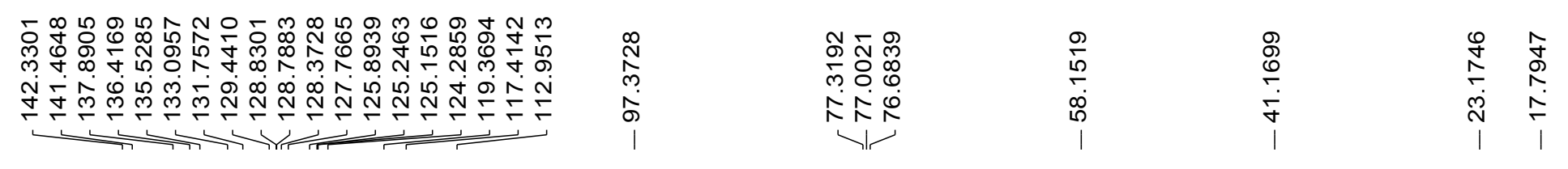
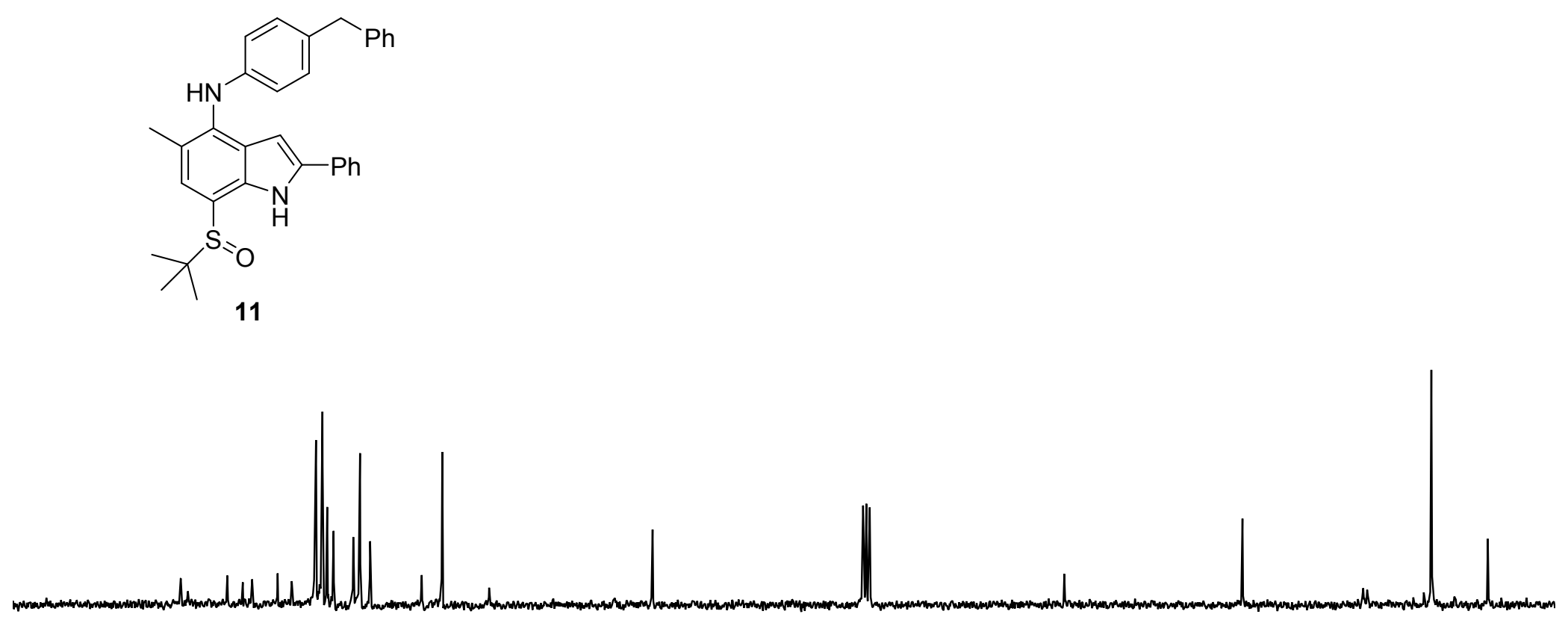

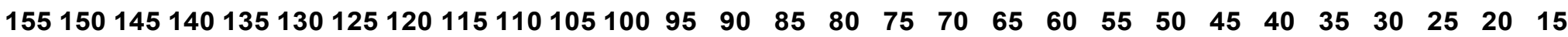
f1 (ppm) 

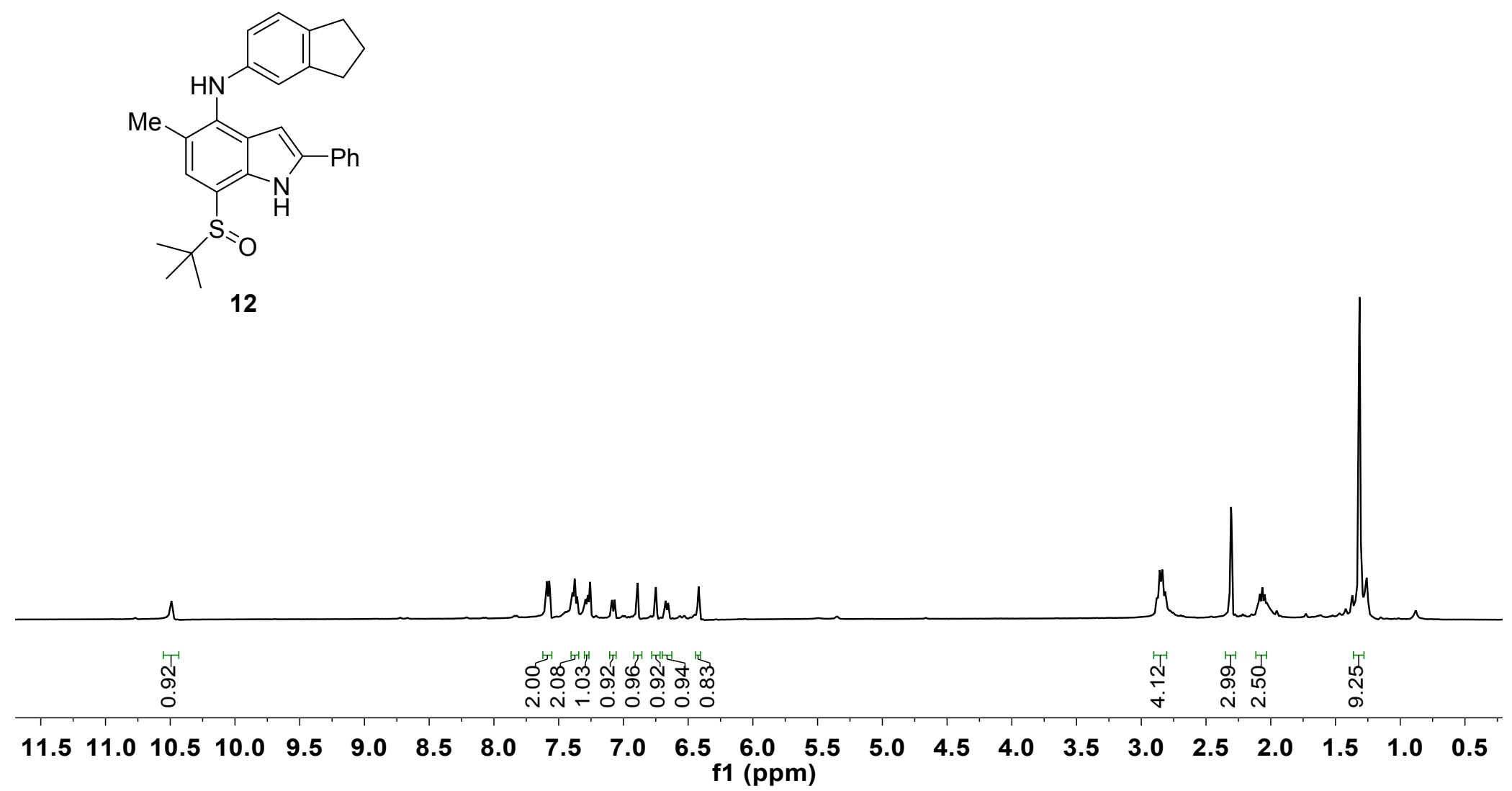


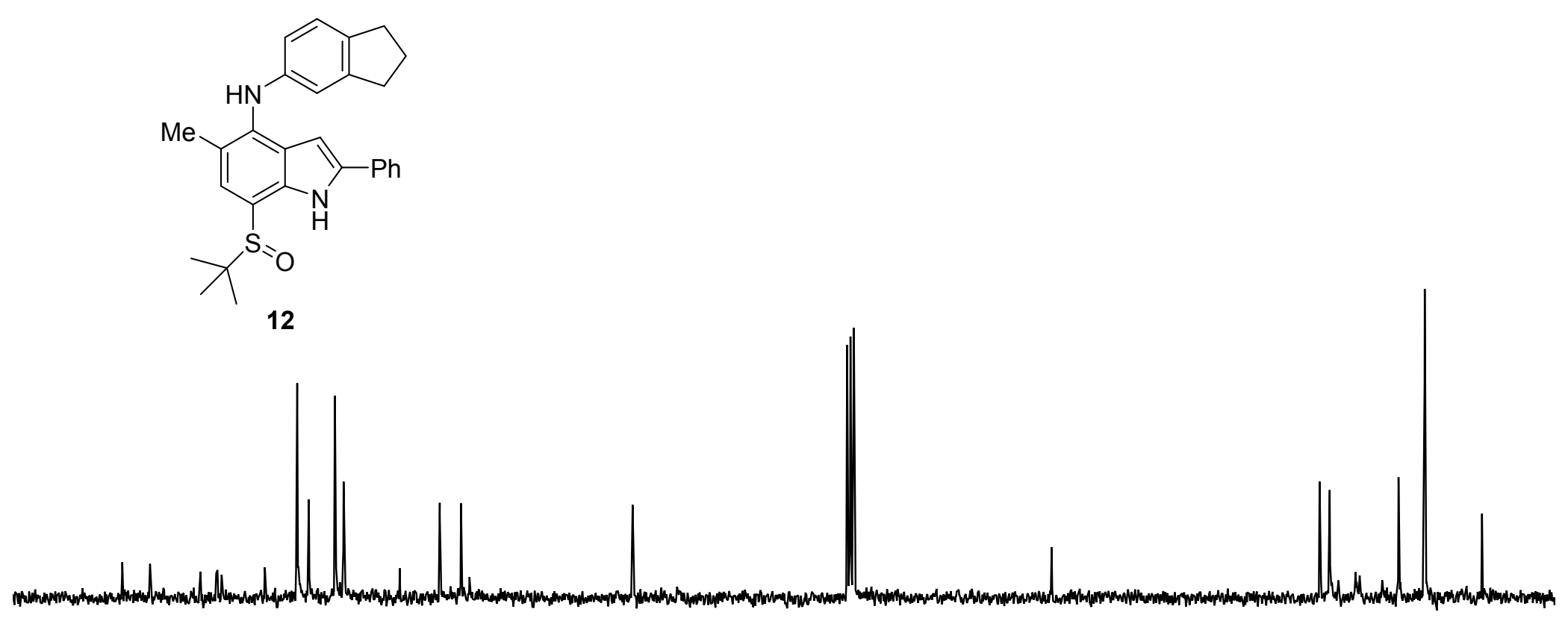

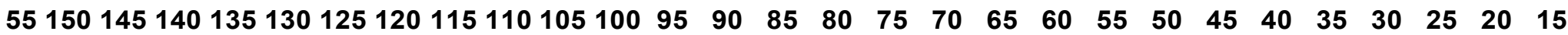
f1 (ppm) 

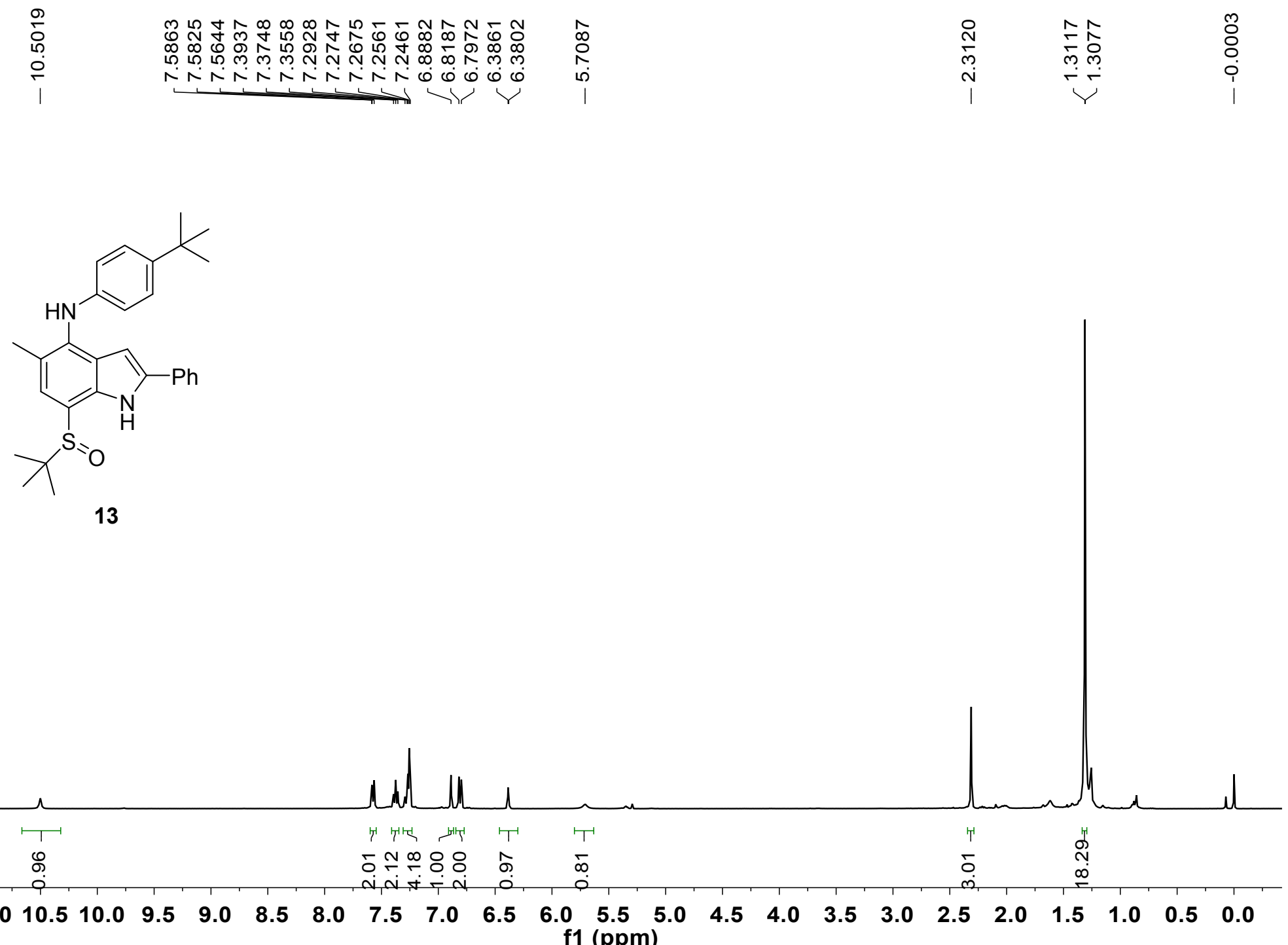

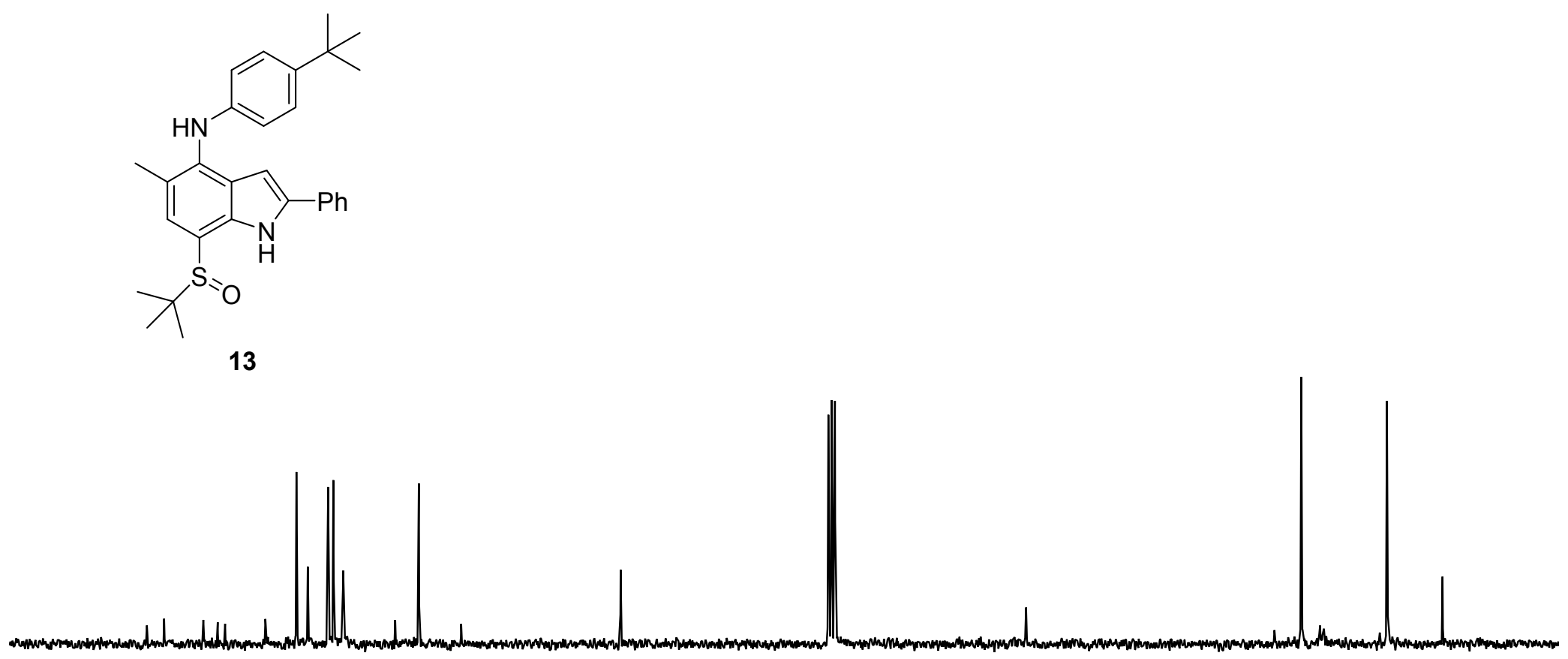

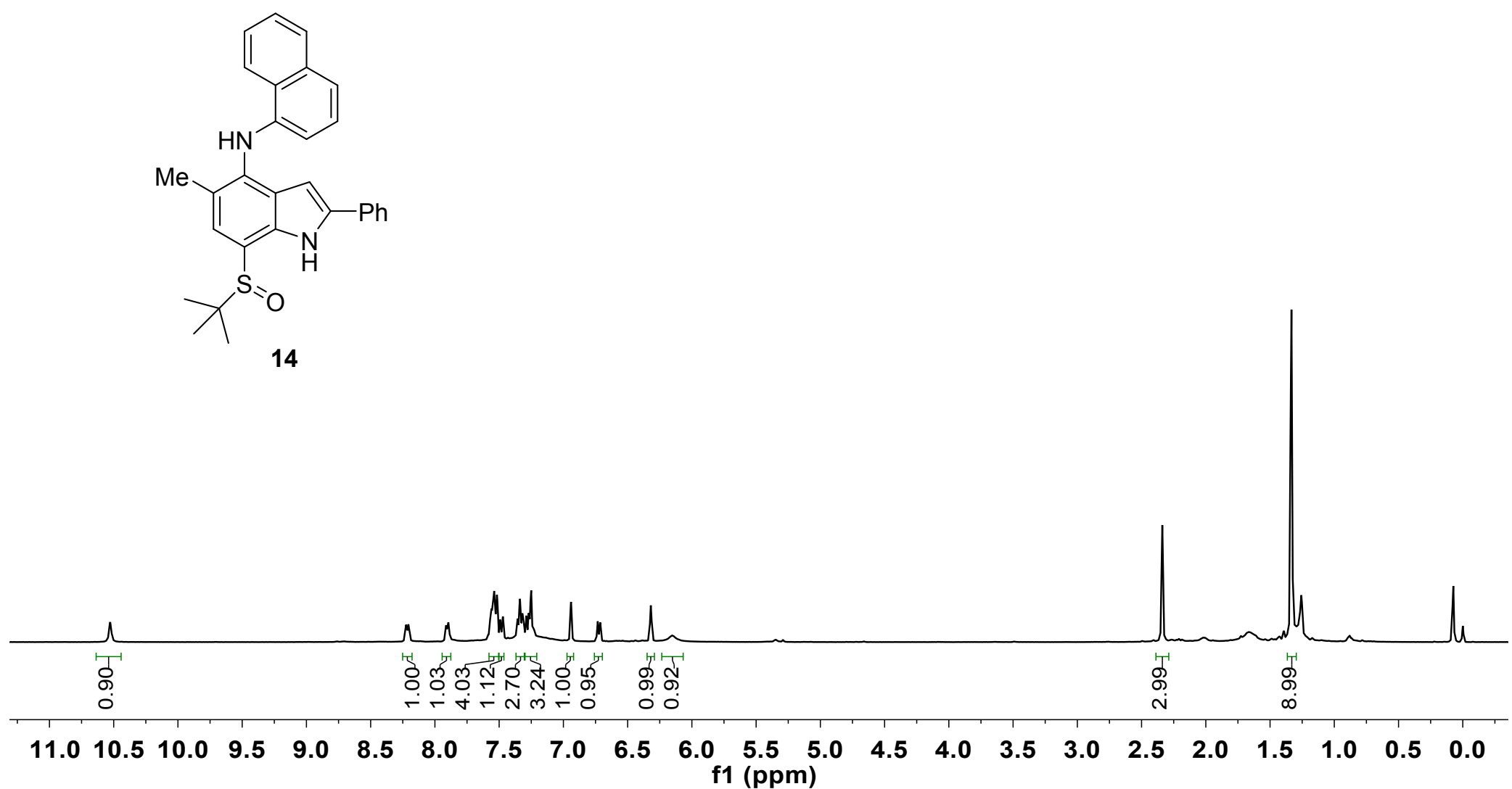

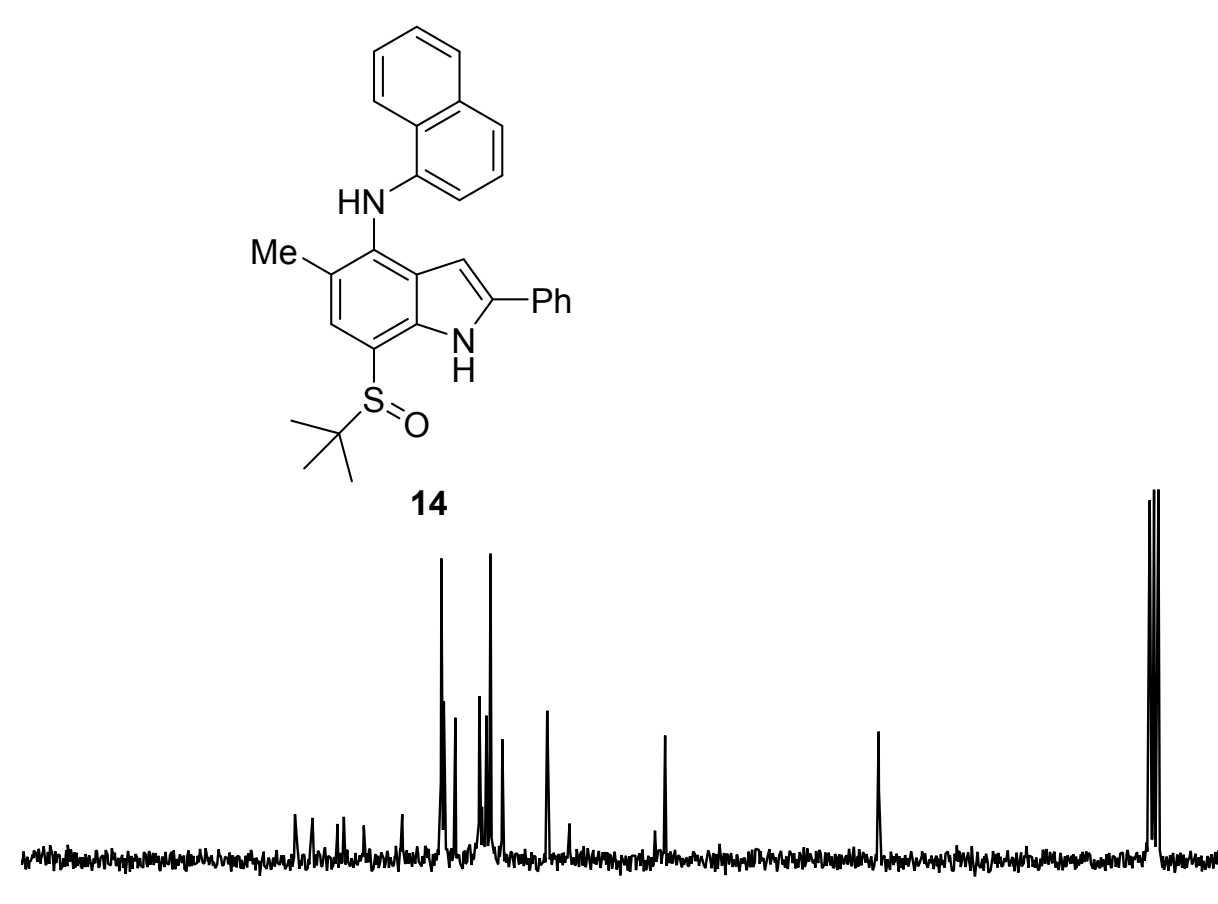

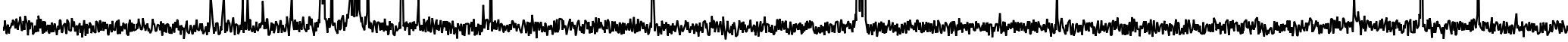




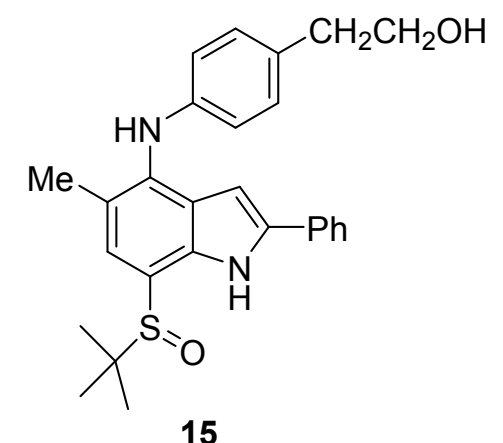

15

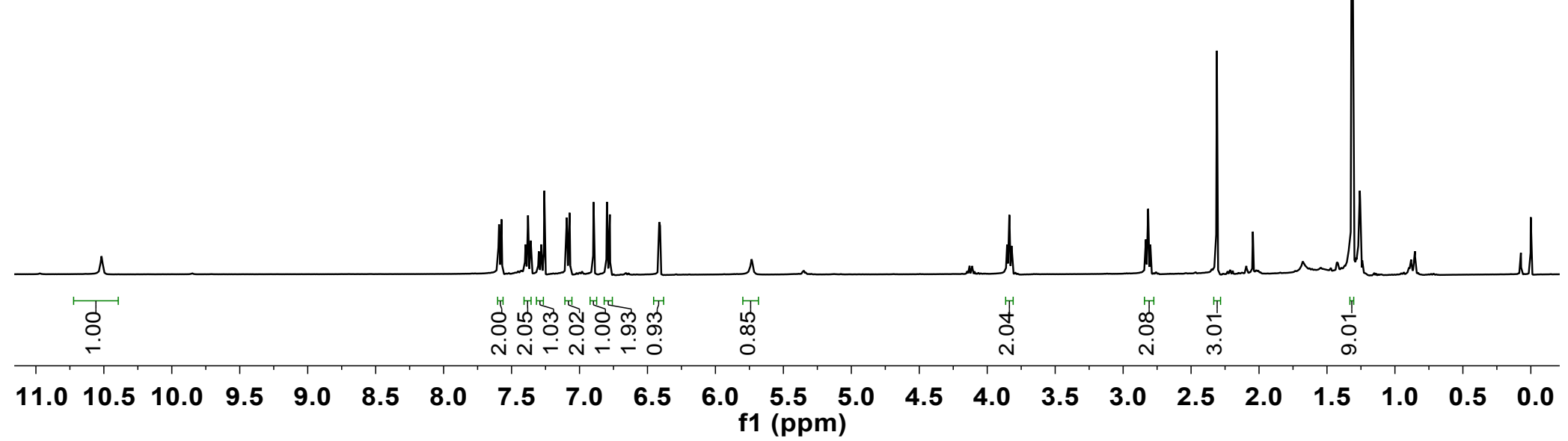




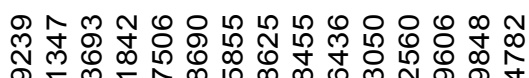

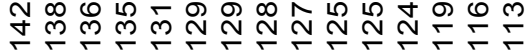
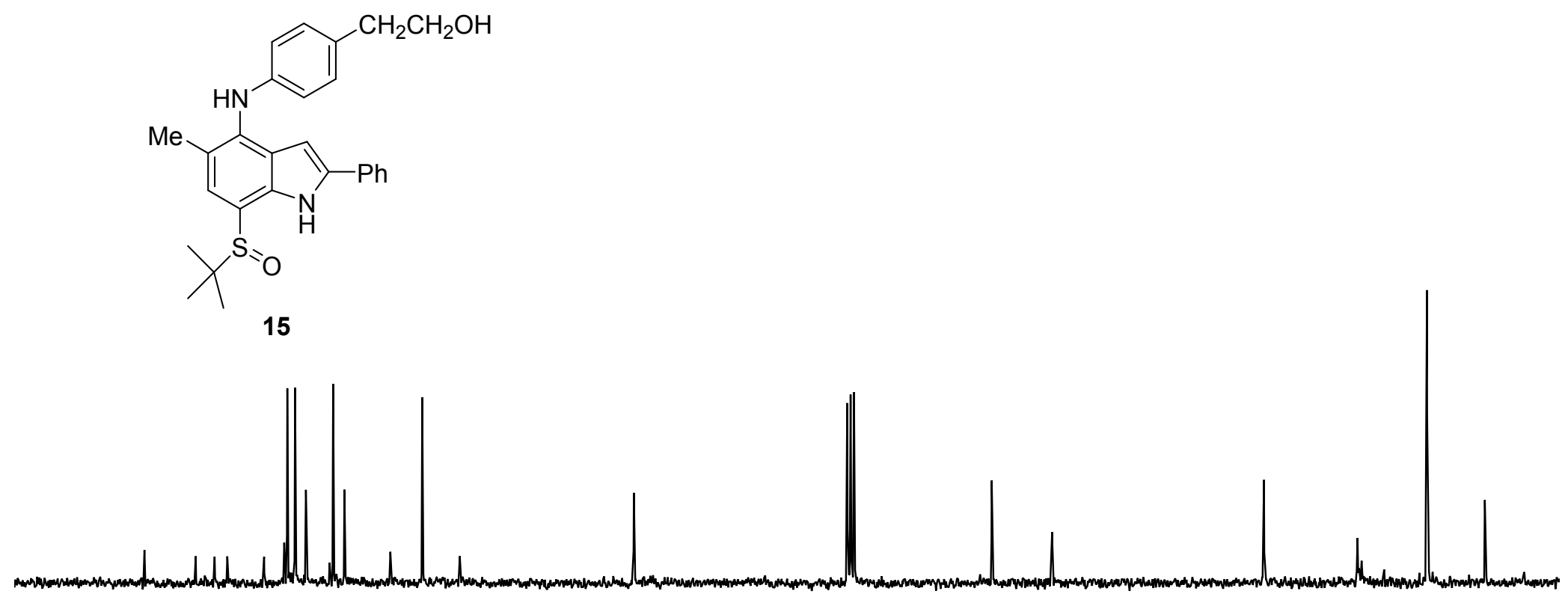

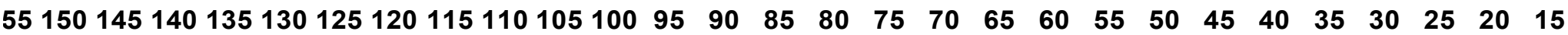
f1 (ppm) 

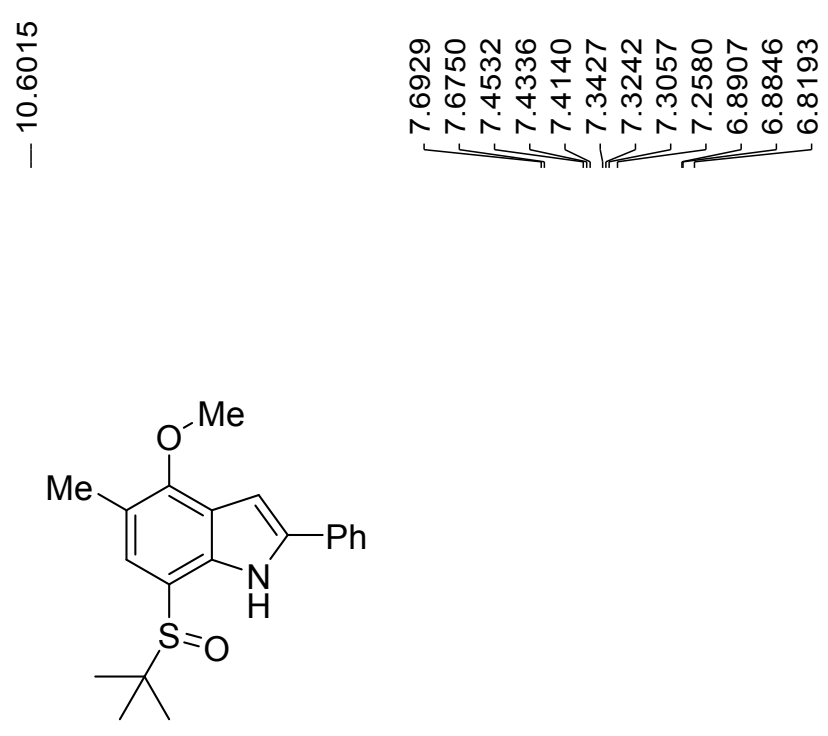

16

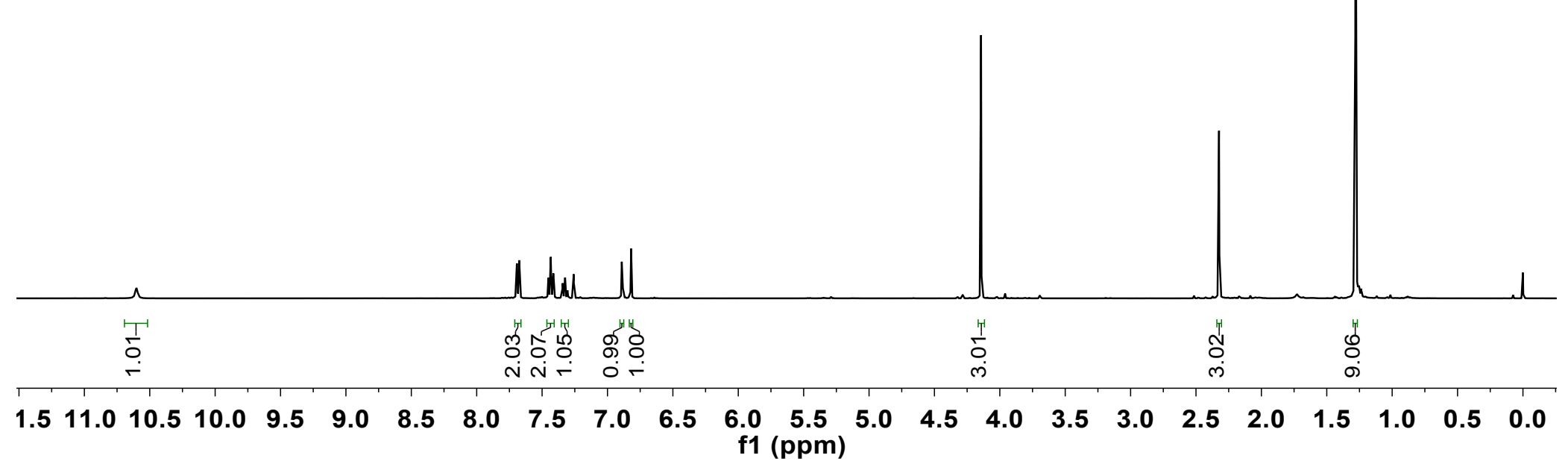



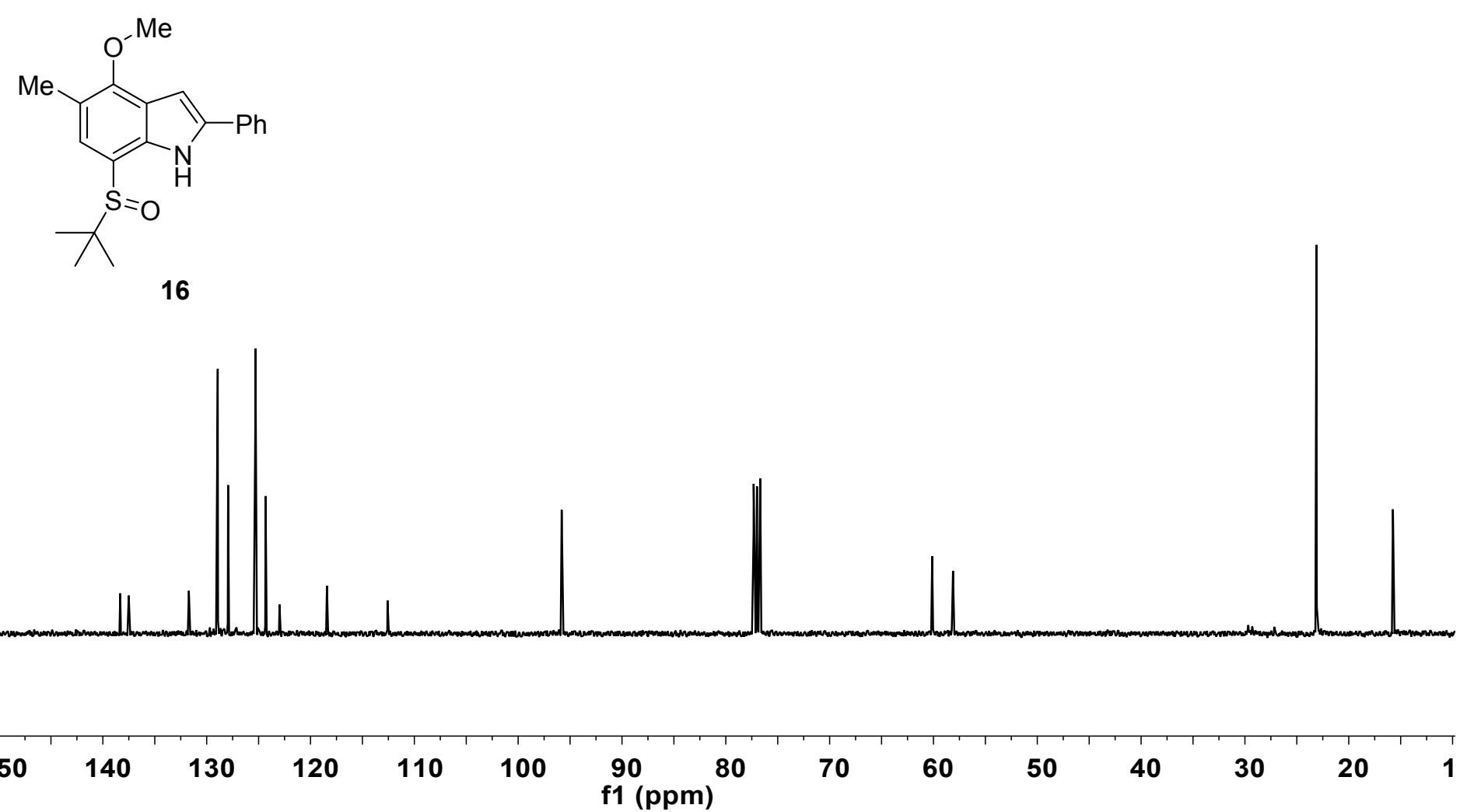

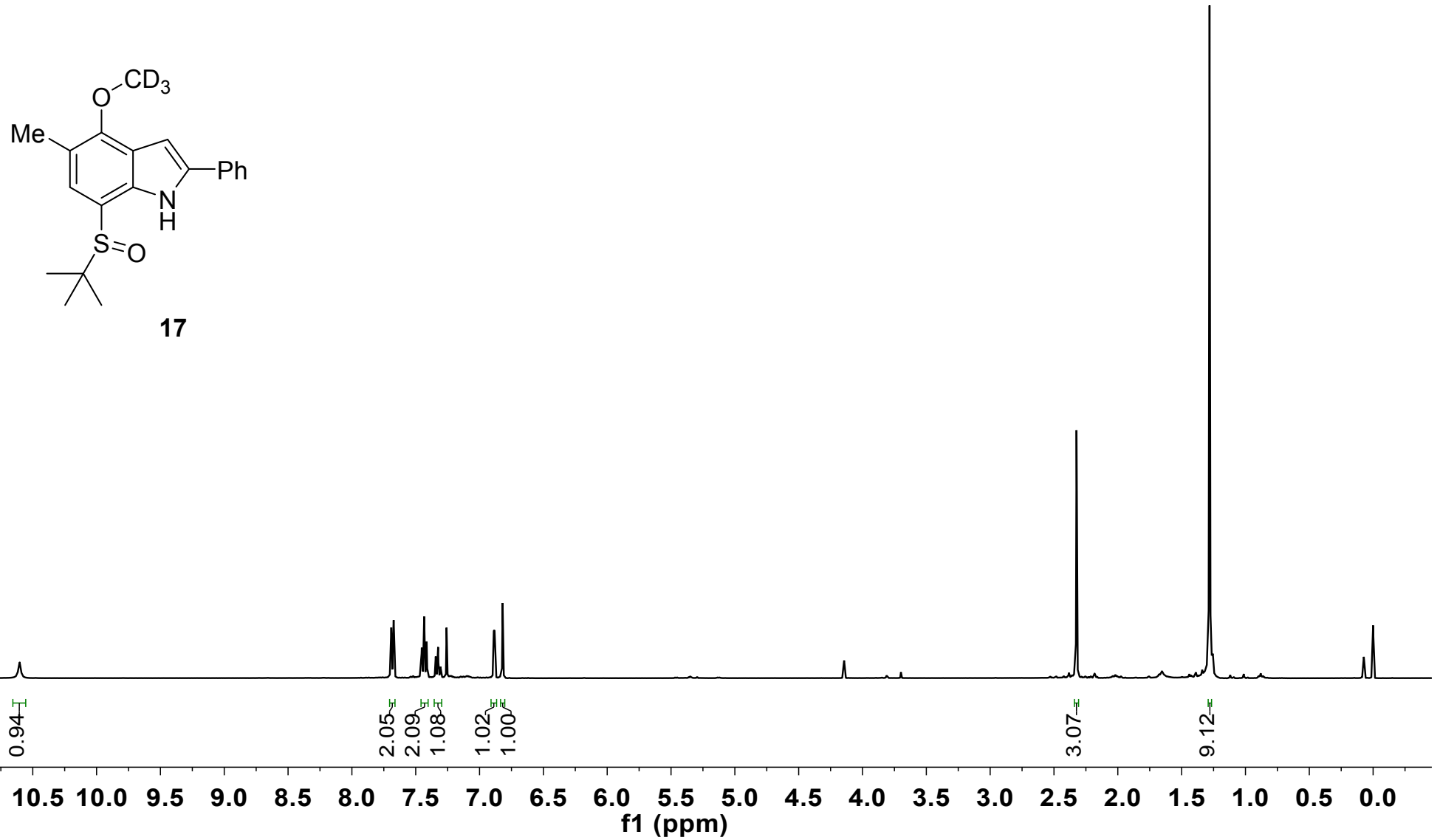

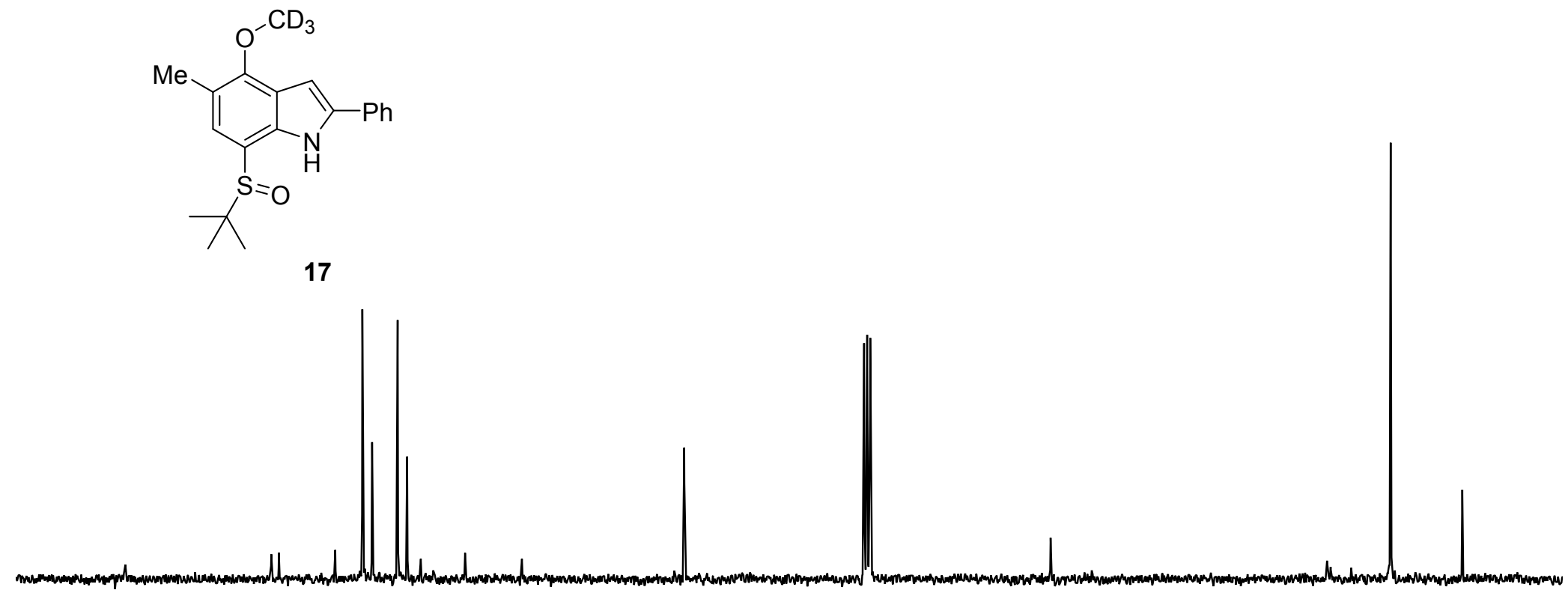


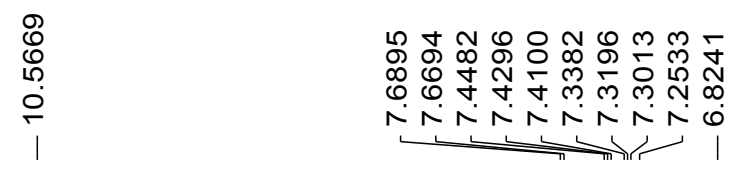

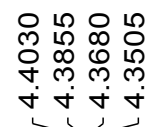

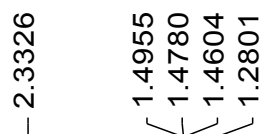
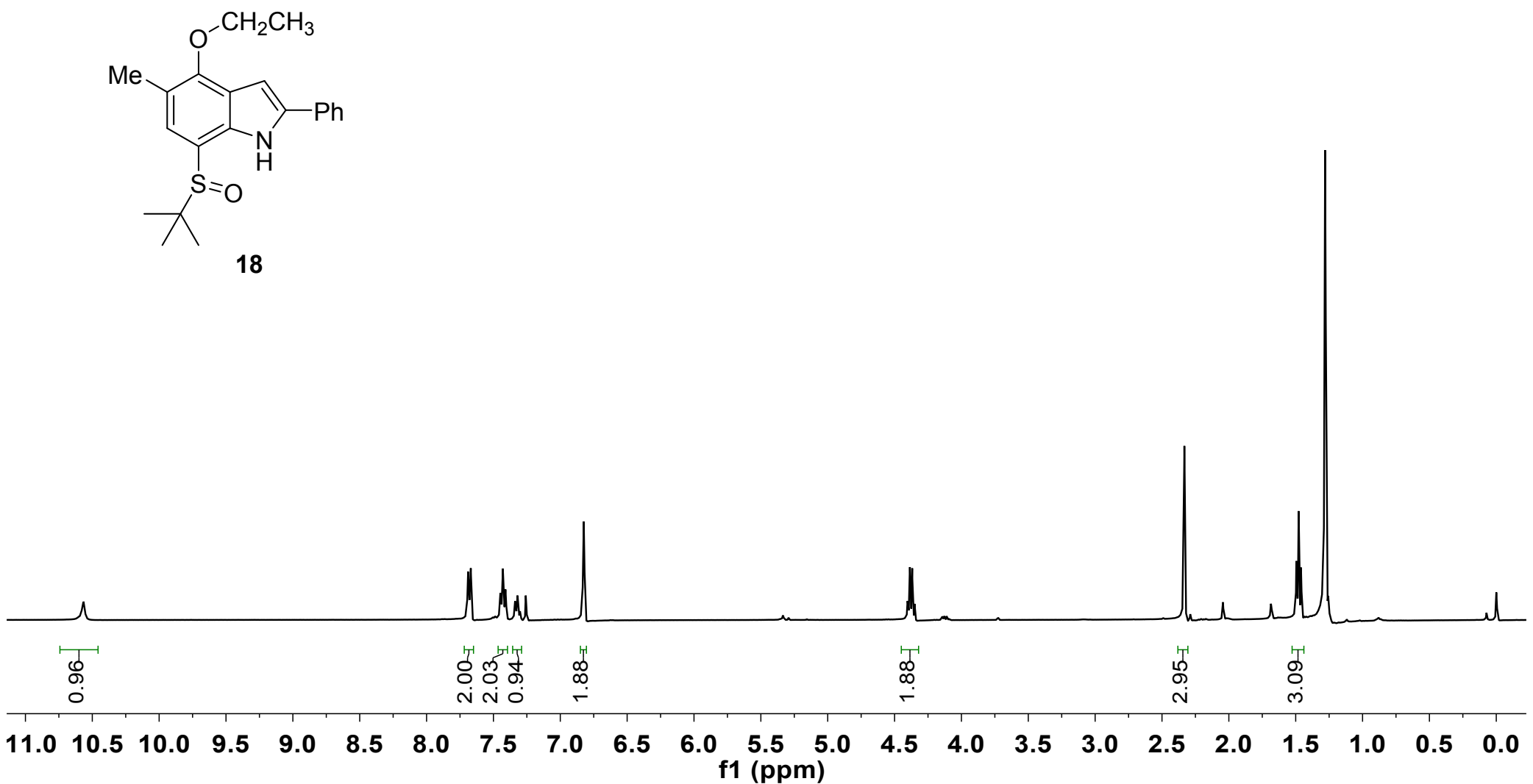
<smiles>CCOc1c(C)cc(S(=O)C(C)(C)C)c2[nH]c(P)cc12</smiles> 

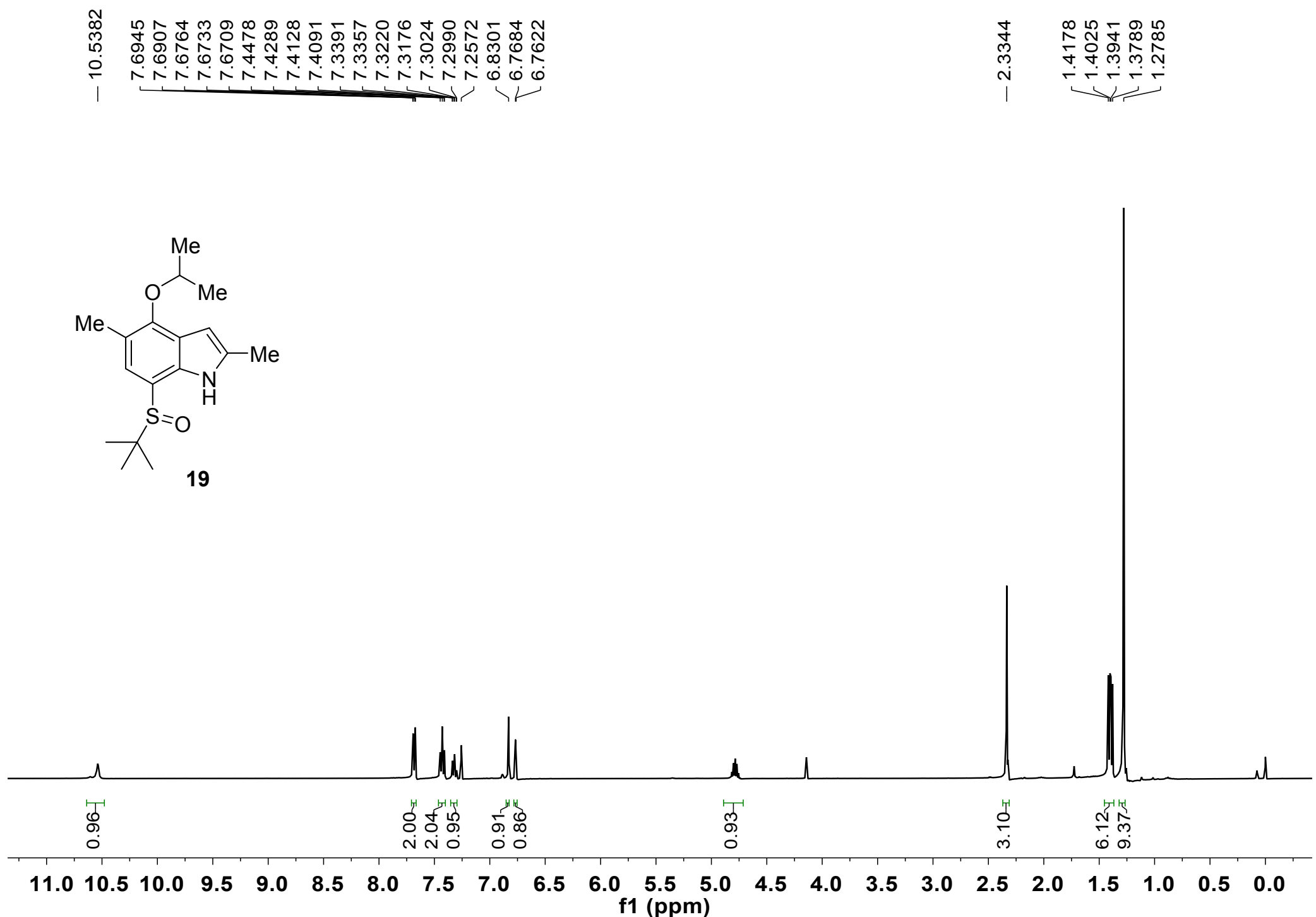

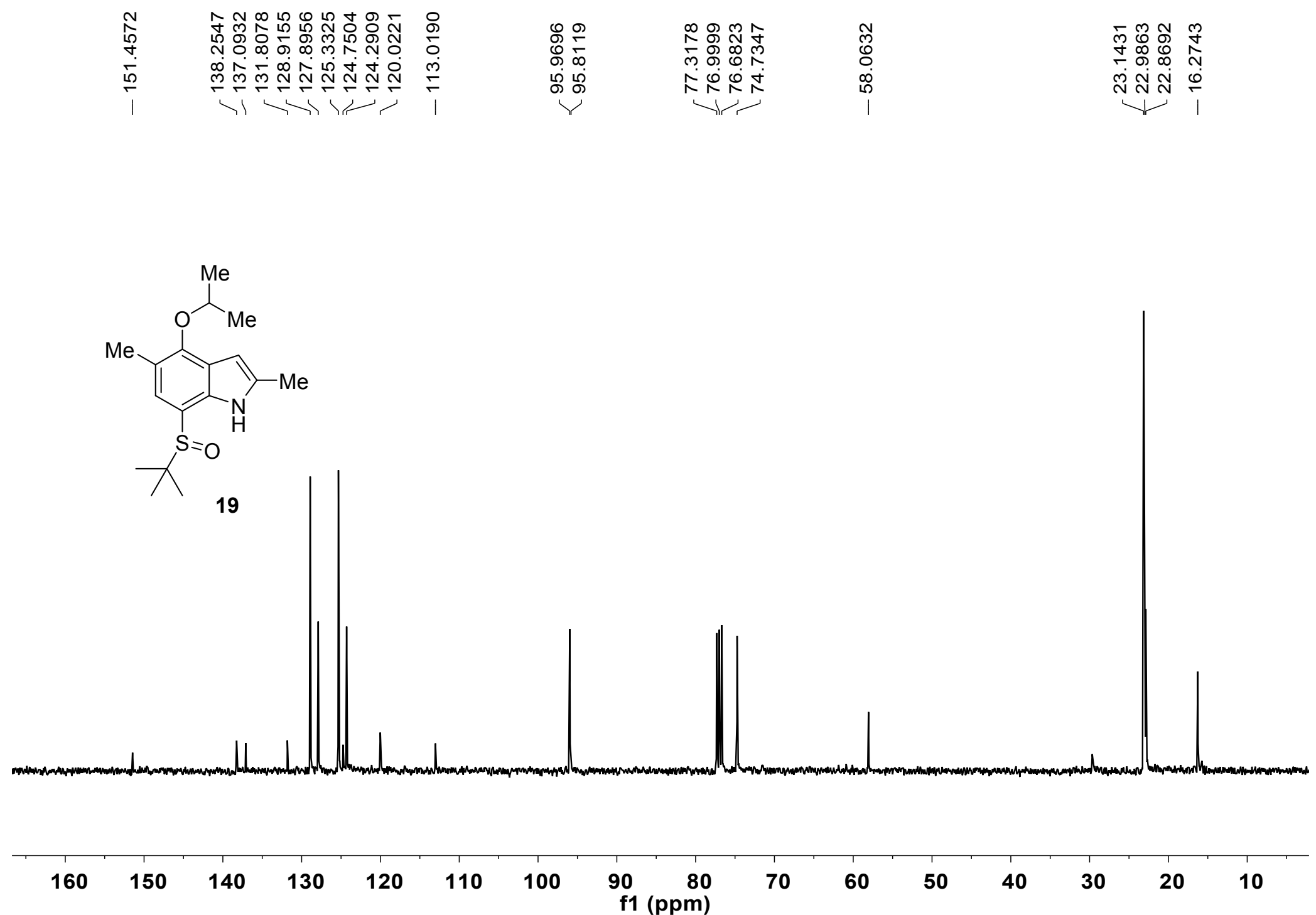

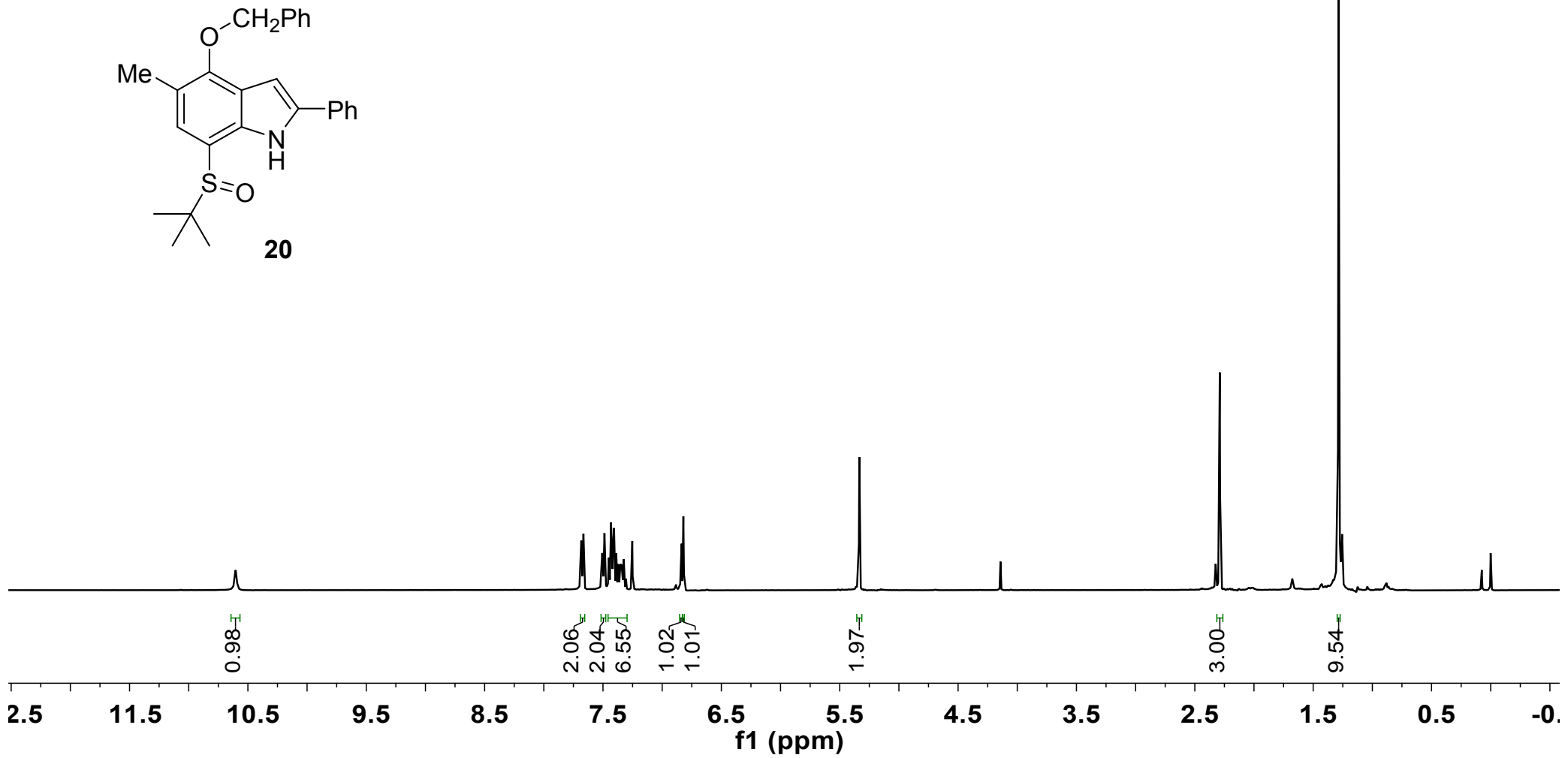

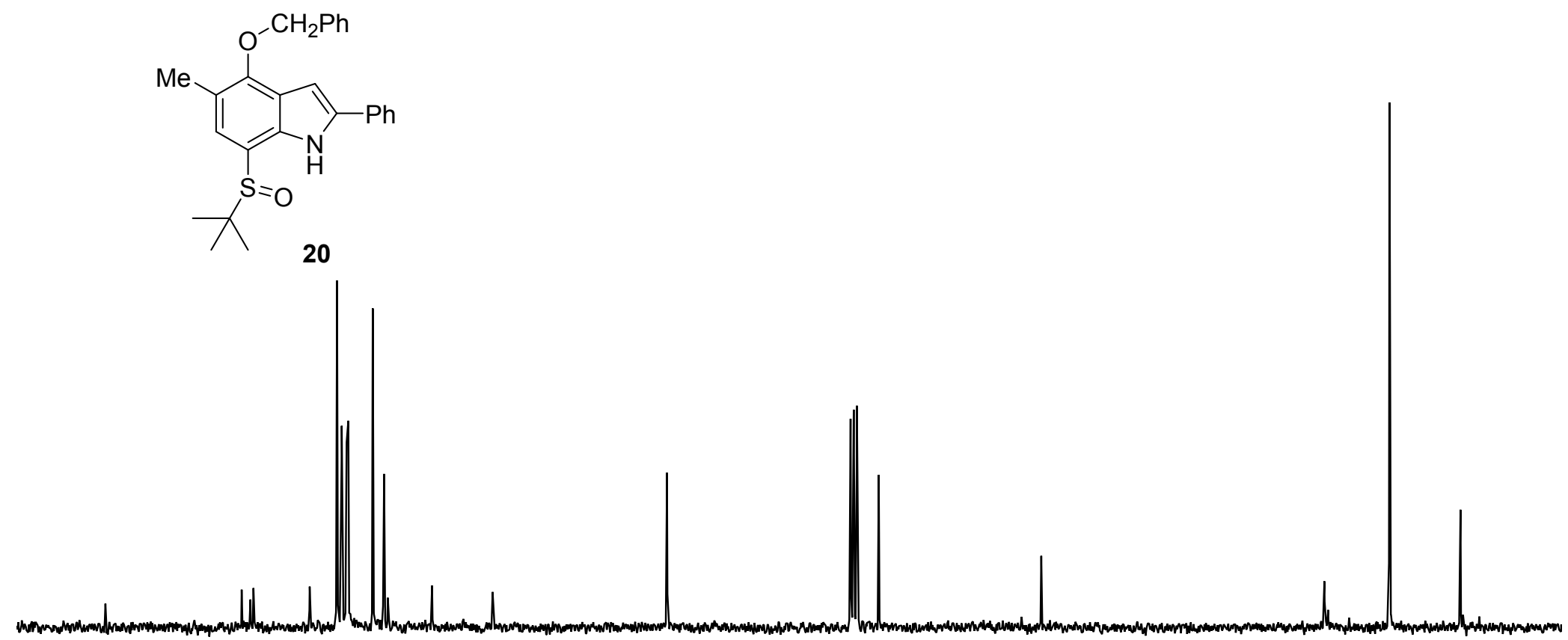

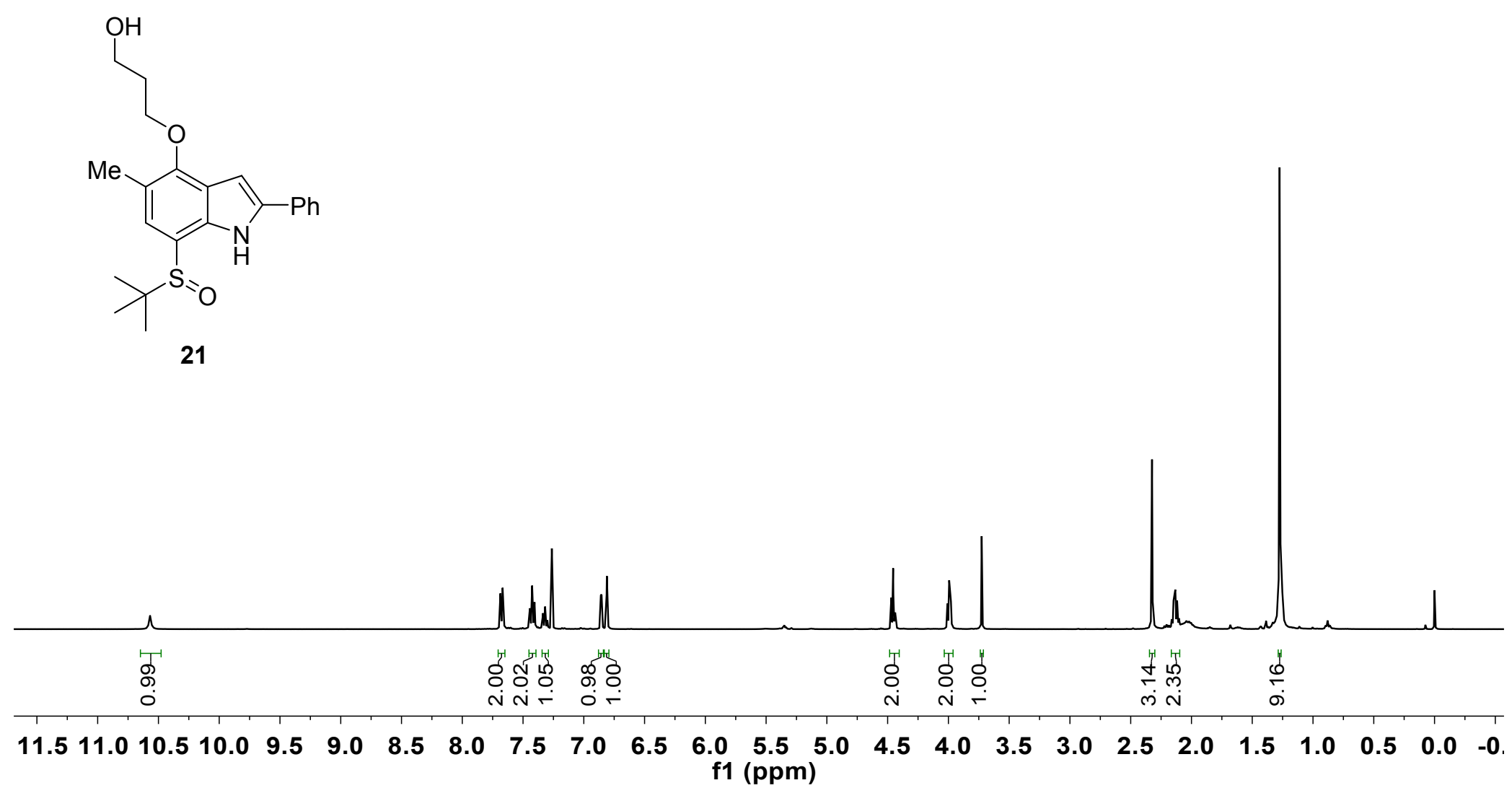


\begin{tabular}{|c|c|c|c|c|c|c|}
\hline $\begin{array}{l}\text { 2. } \\
\text { } \\
\text { v } \\
\end{array}$ & 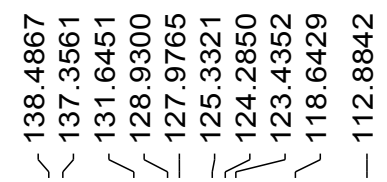 & 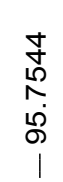 & 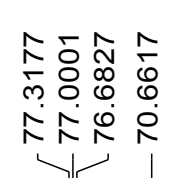 & 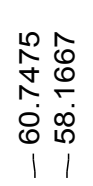 & $\begin{array}{c}\hat{N} \\
\hat{0} \\
\mathbb{m}^{2} \\
1\end{array}$ & 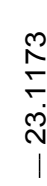 \\
\hline
\end{tabular}
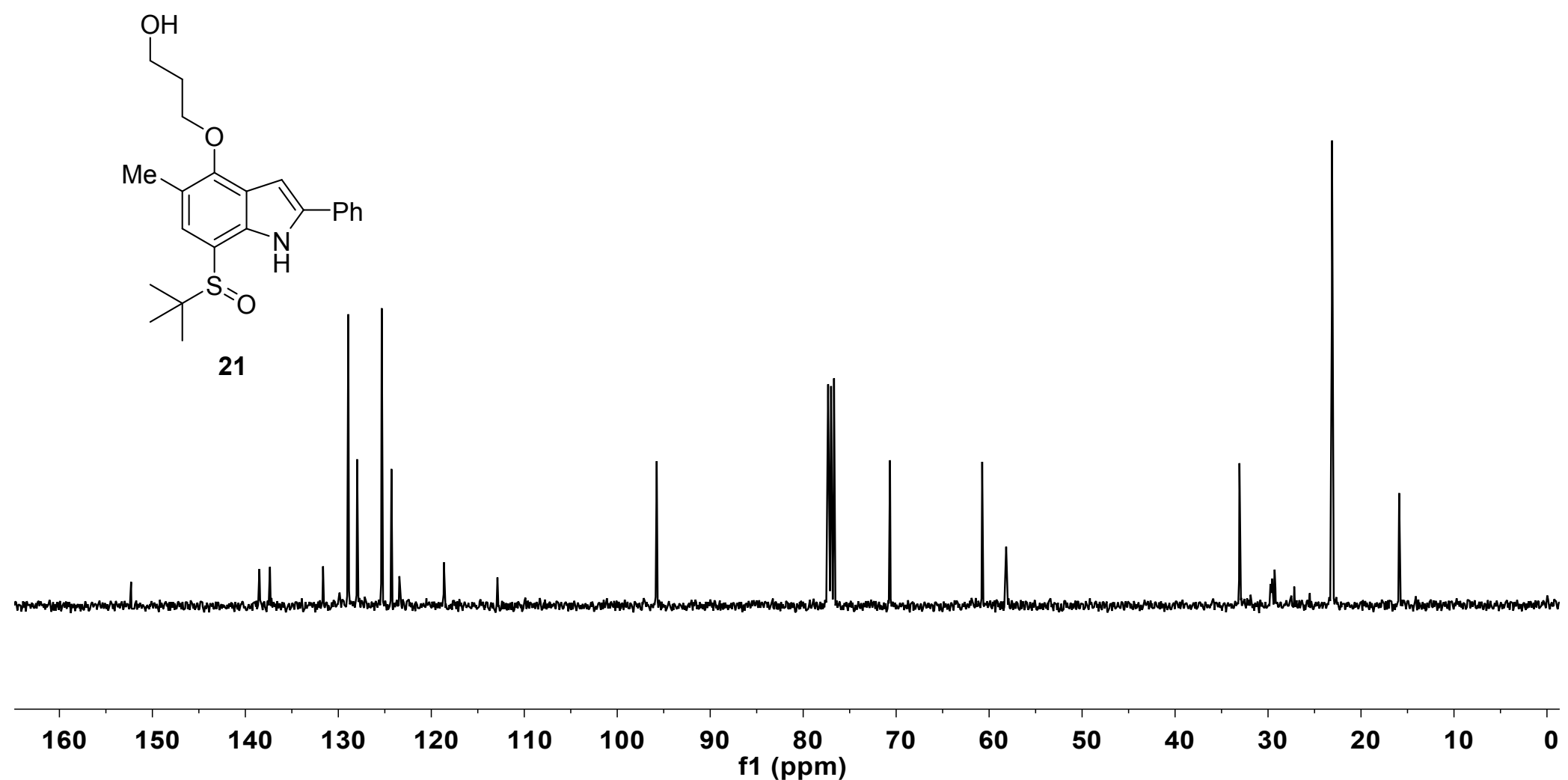

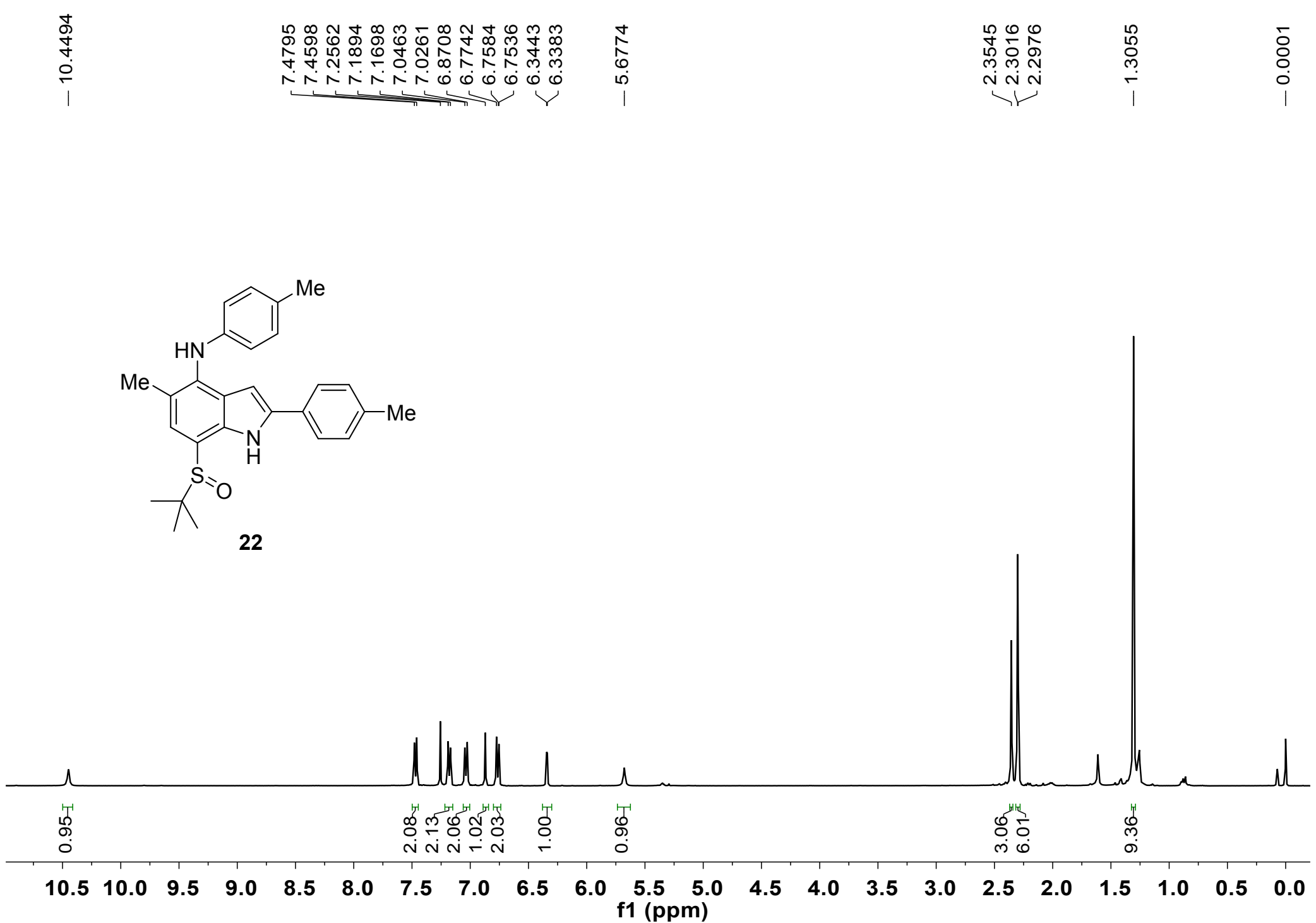


\begin{tabular}{|c|c|c|c|}
\hline 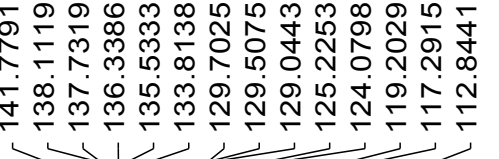 & 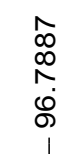 & 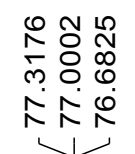 & 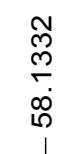 \\
\hline
\end{tabular}

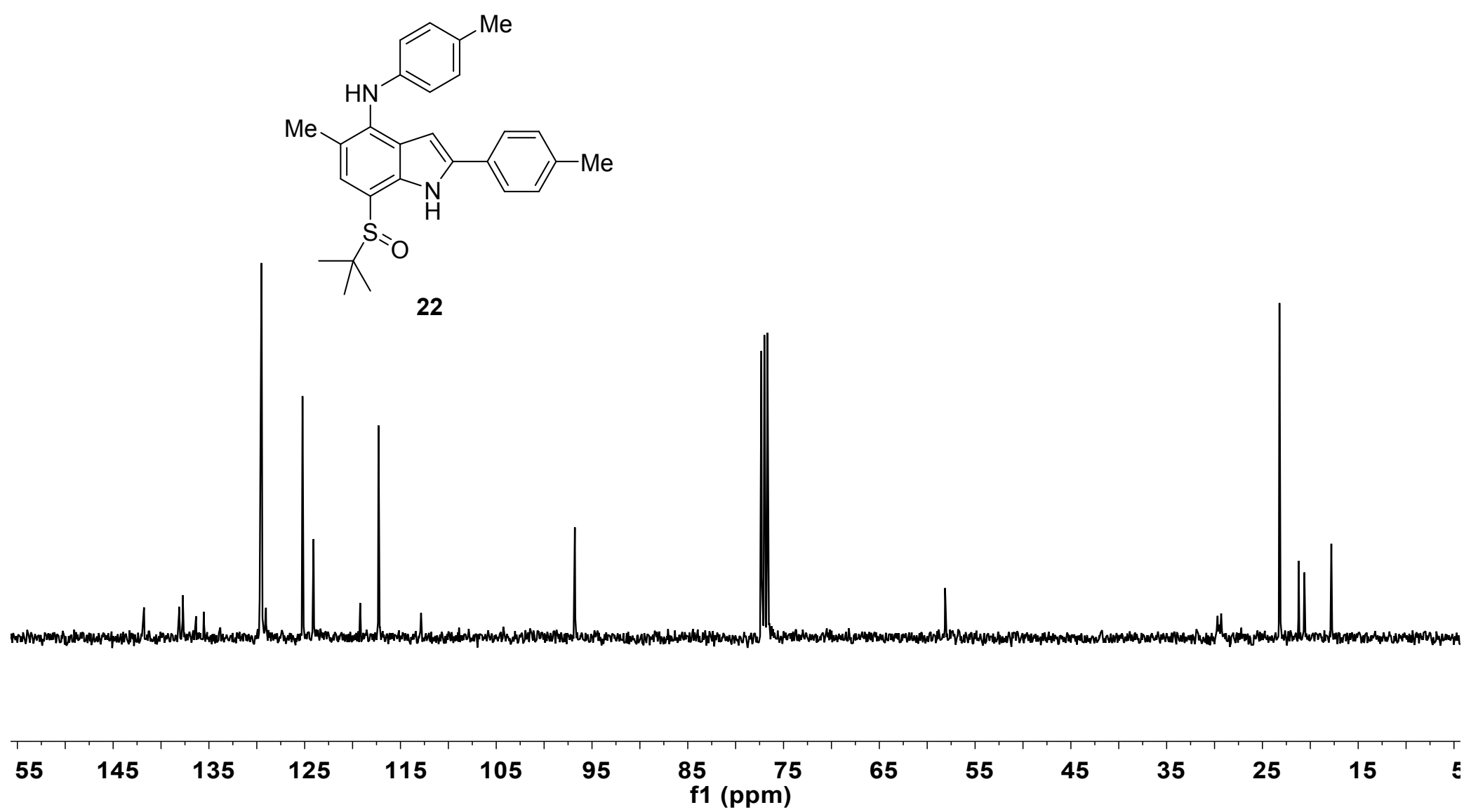



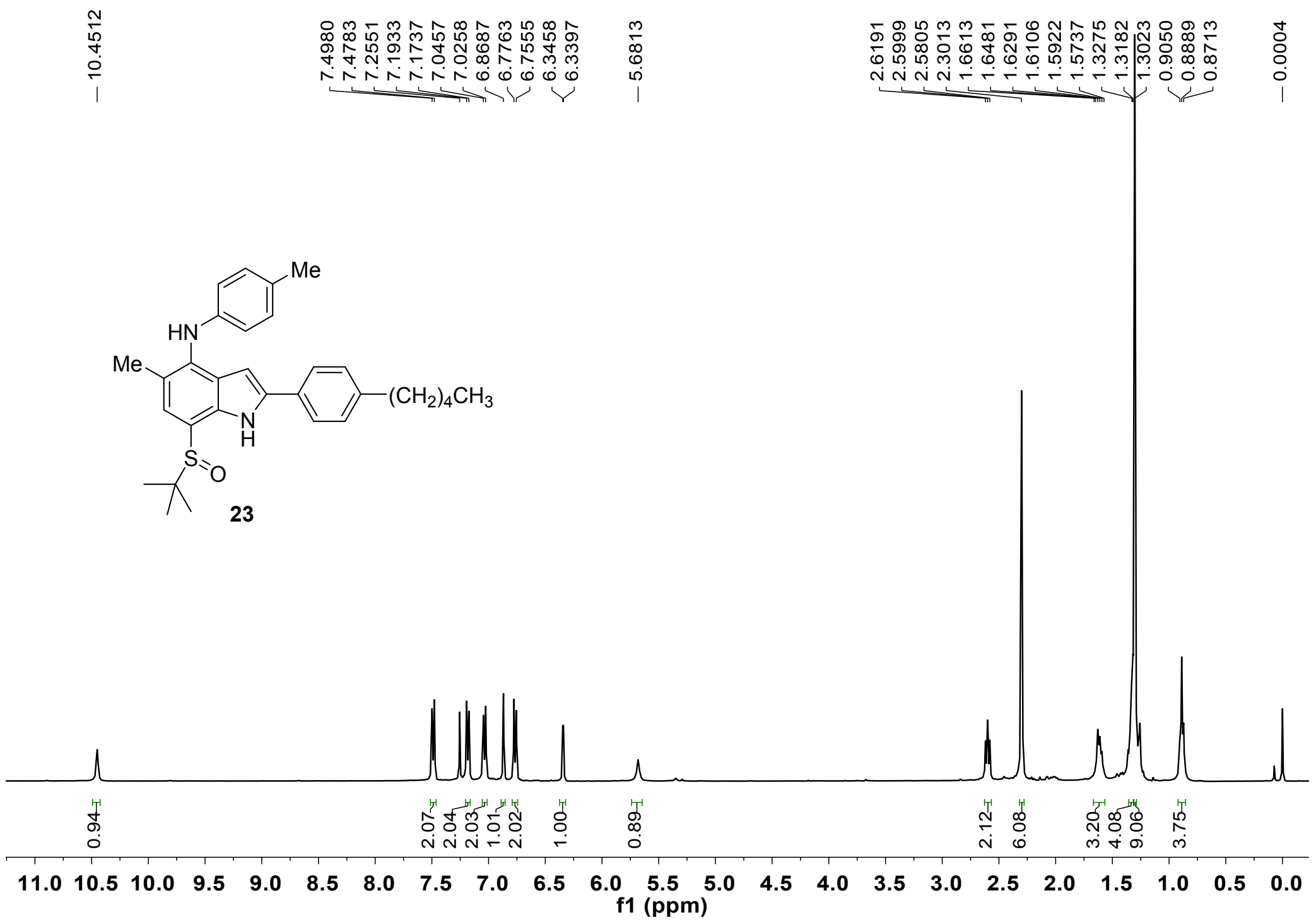


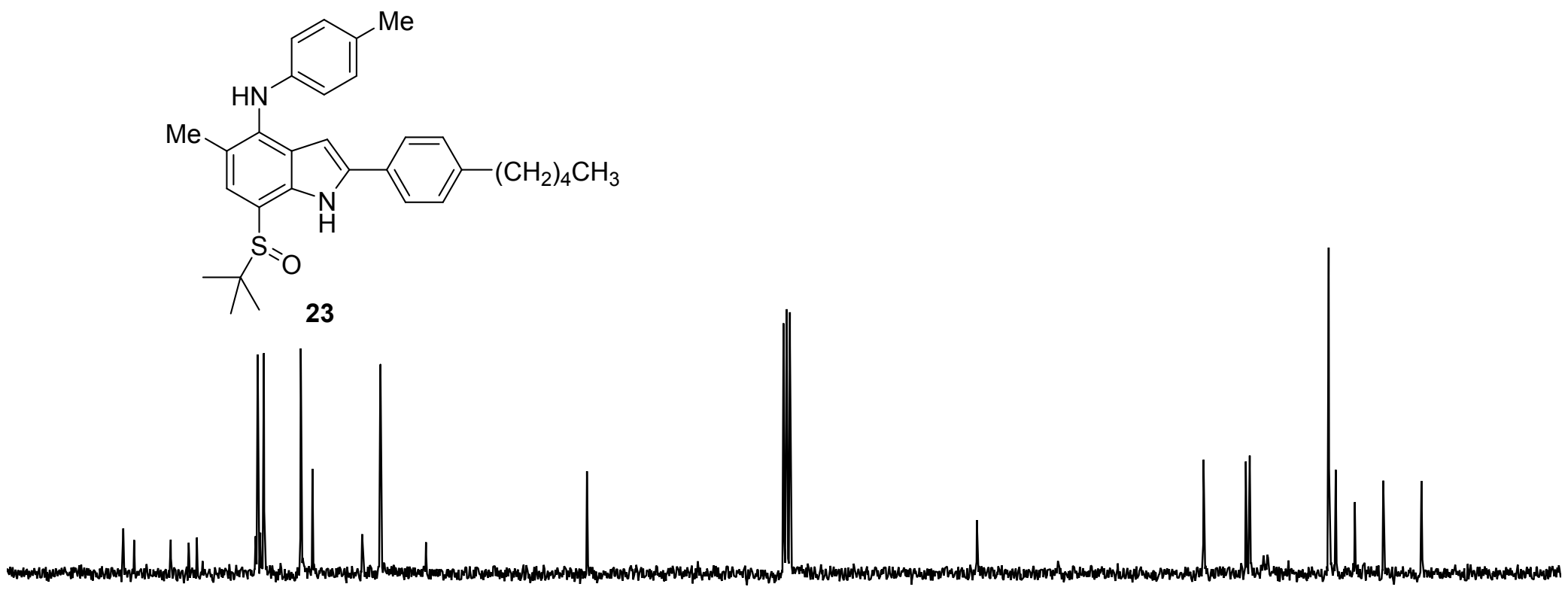



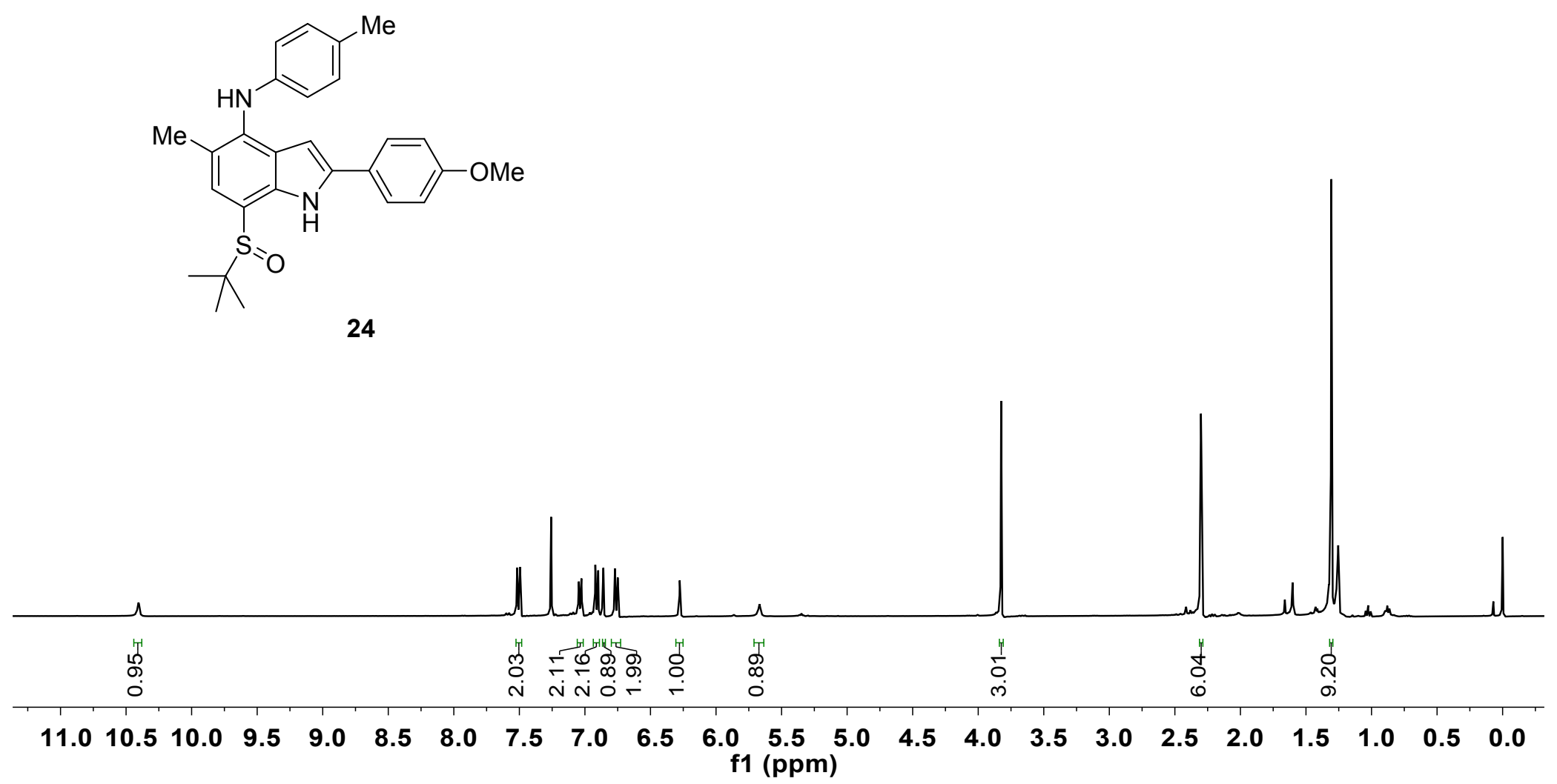


\begin{tabular}{|c|c|c|c|}
\hline 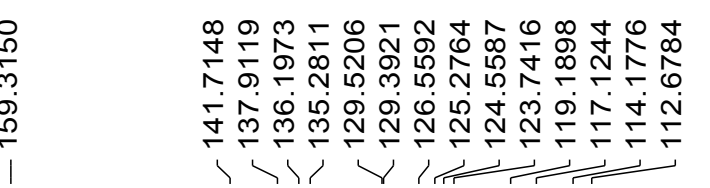 & 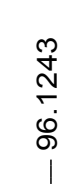 & 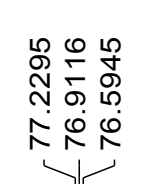 & 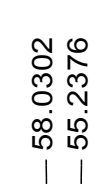 \\
\hline
\end{tabular}
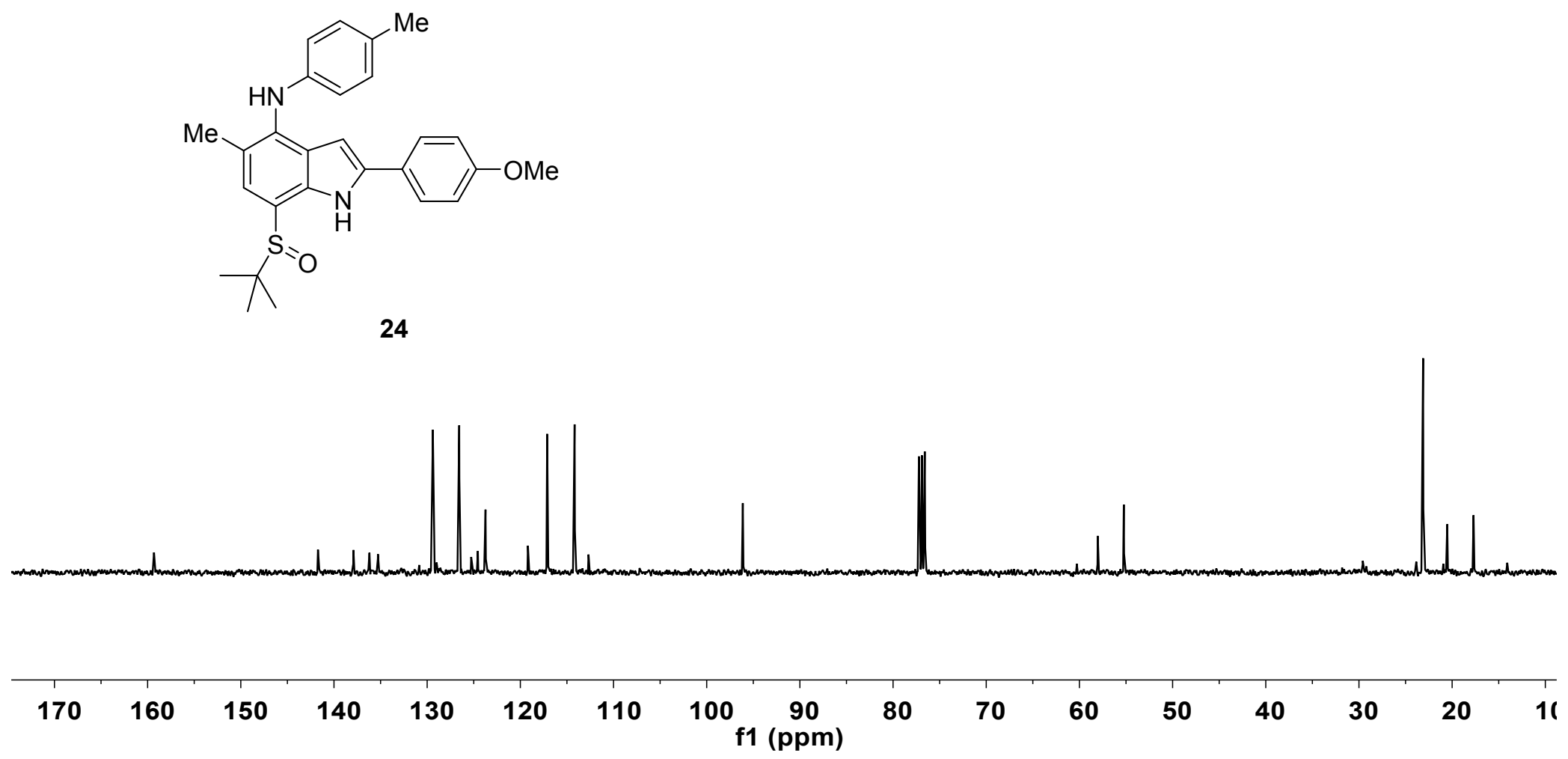


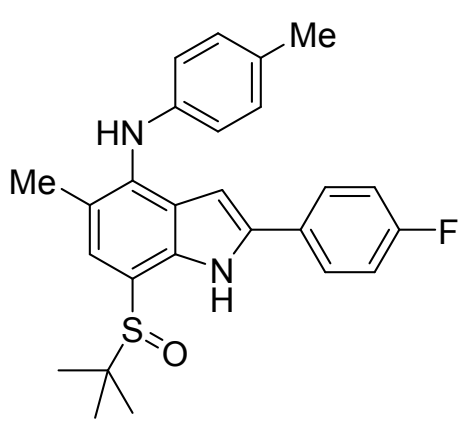

25

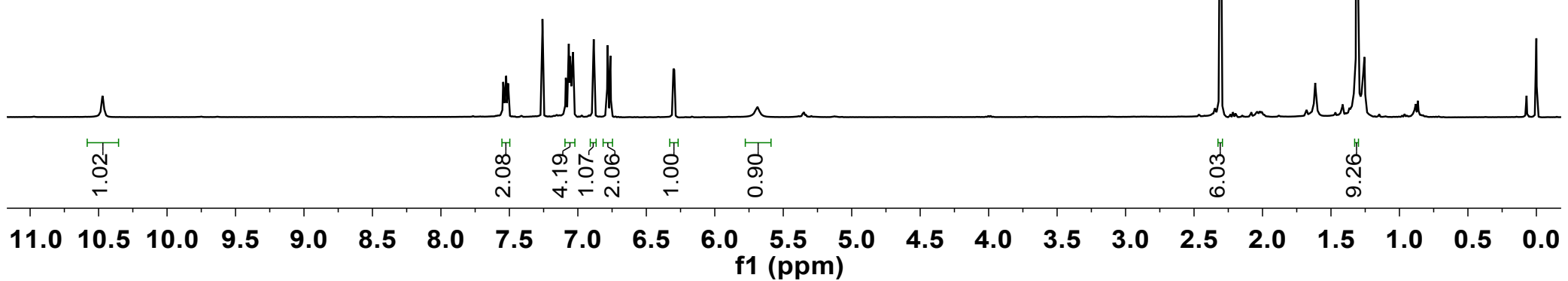



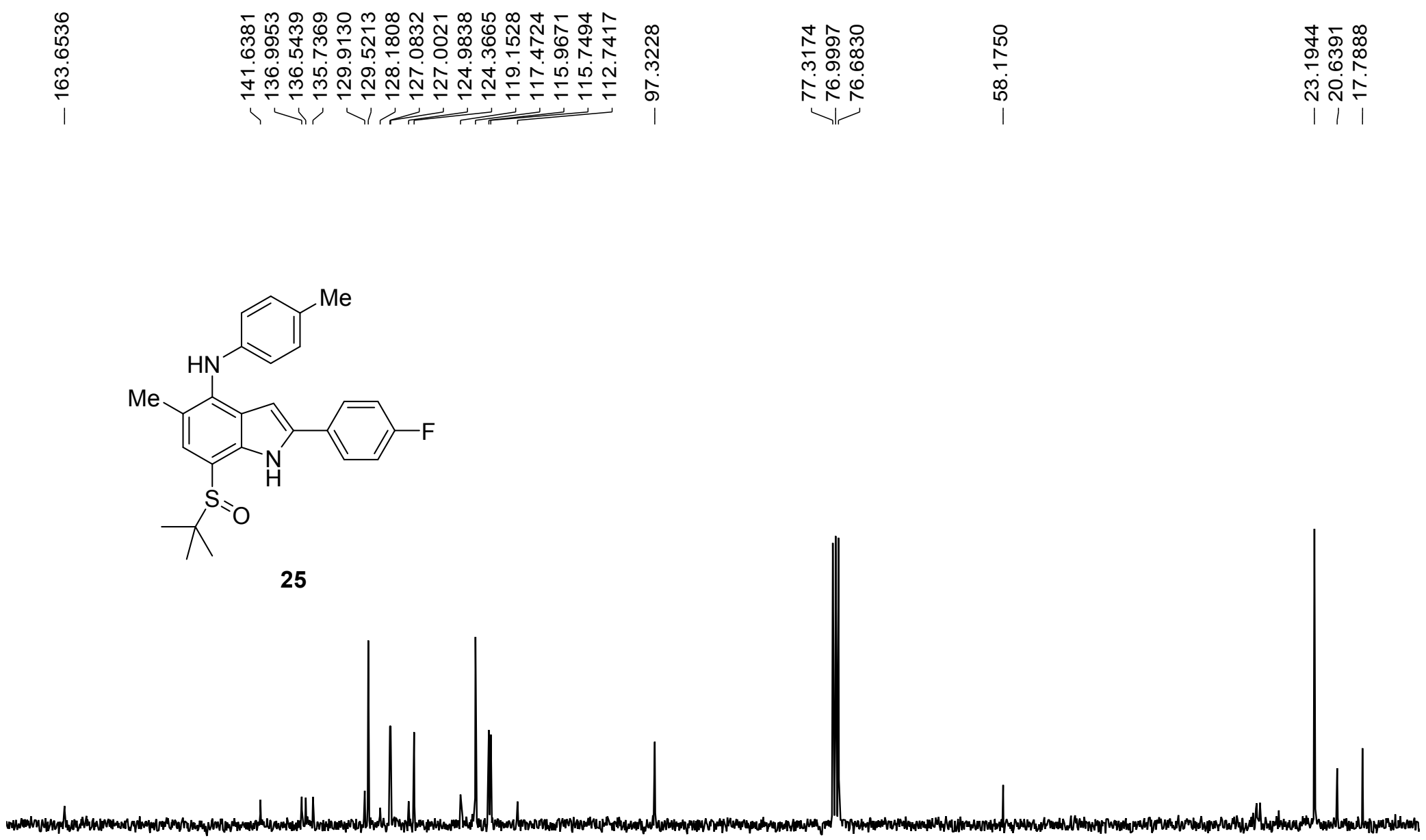

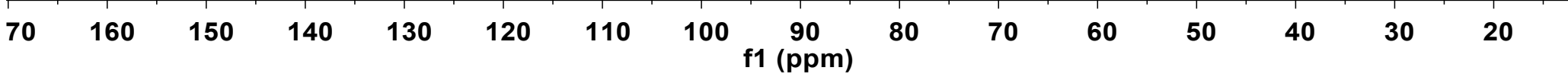



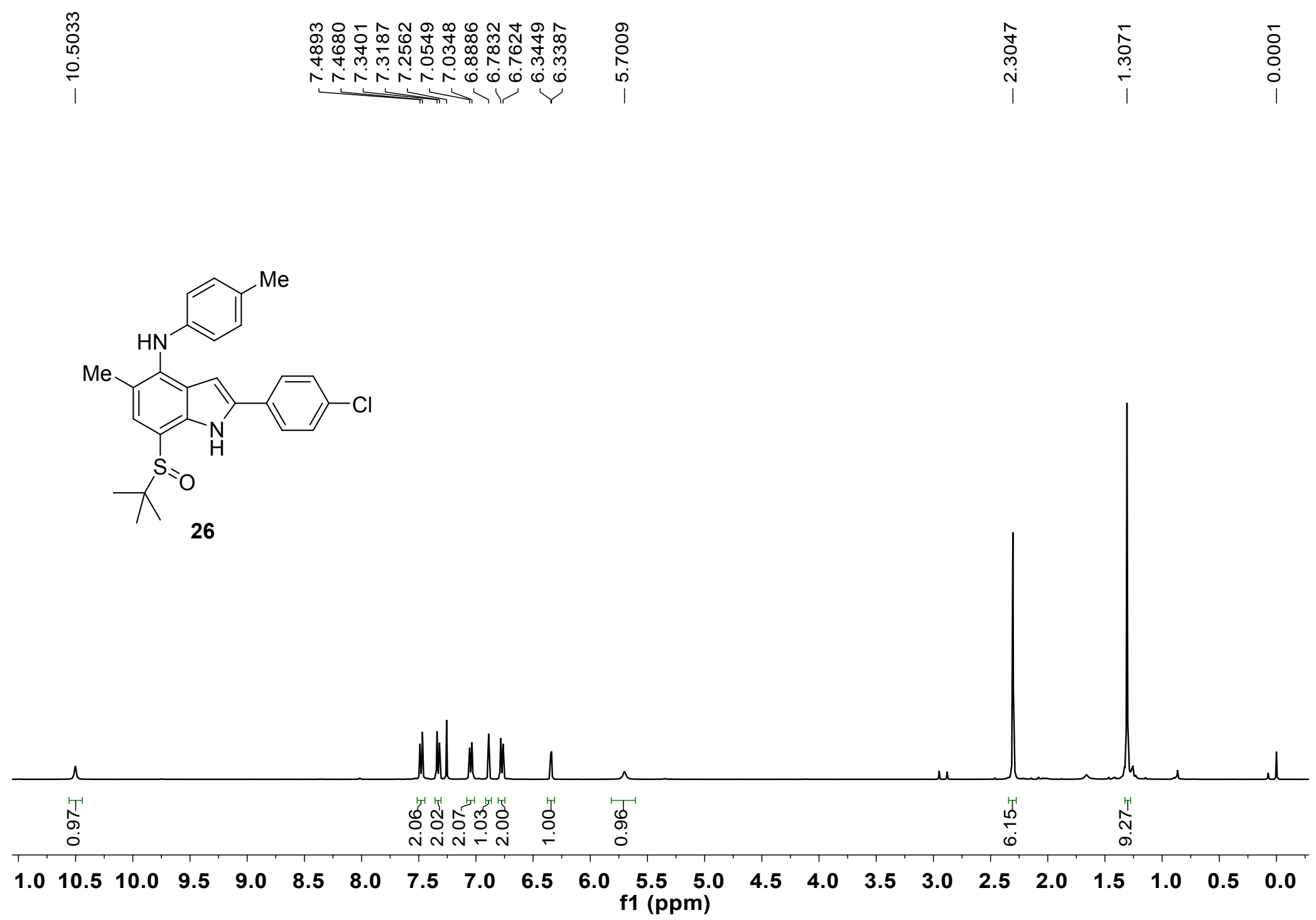


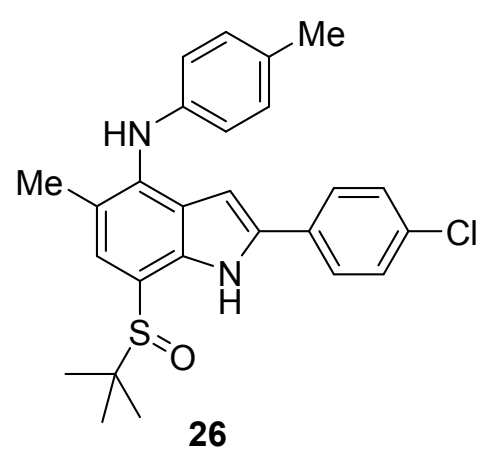

\section{5}

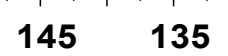

125

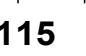

105

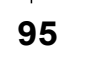

85 f1 (ppm)

65

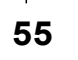

45 

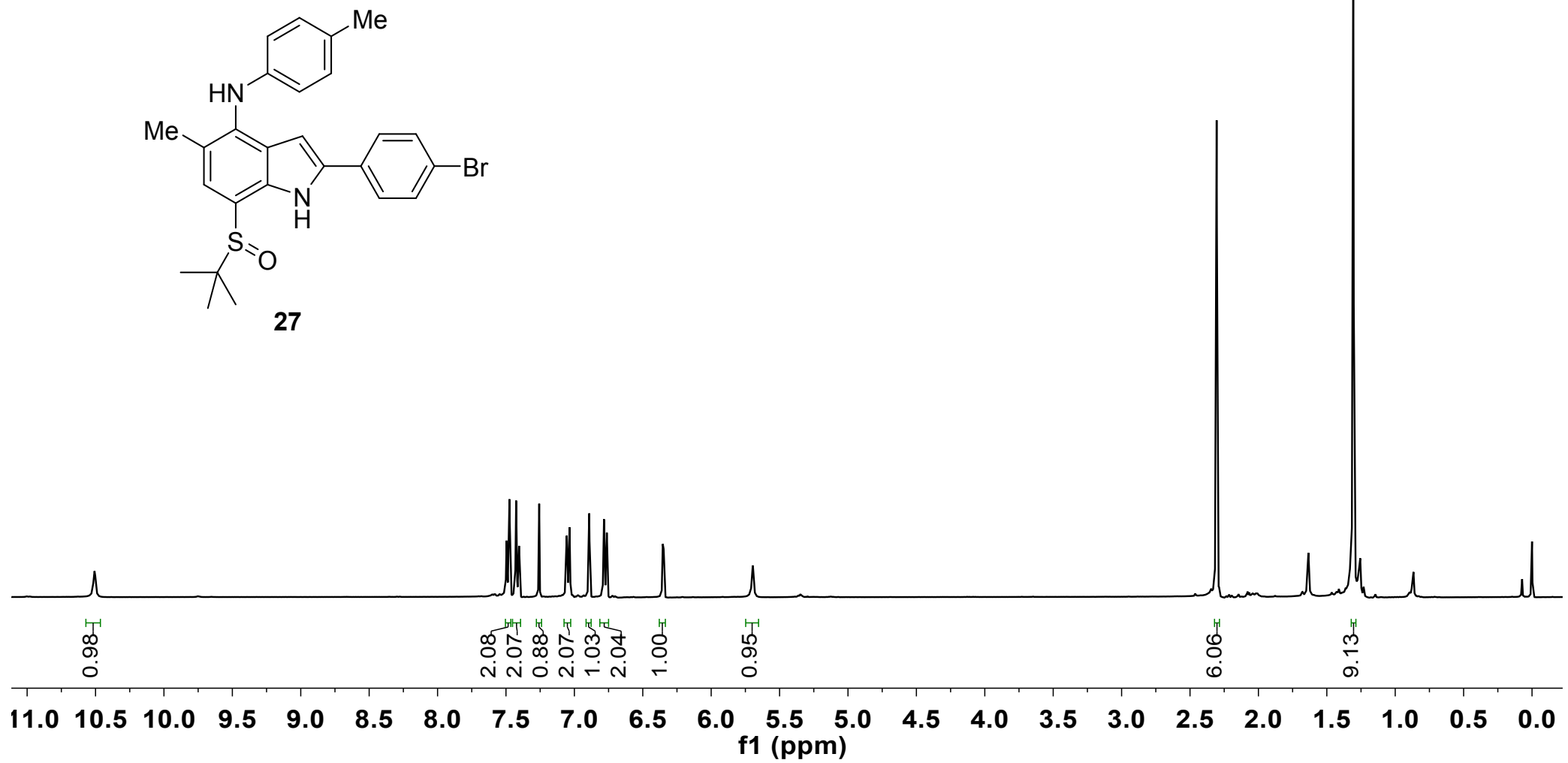

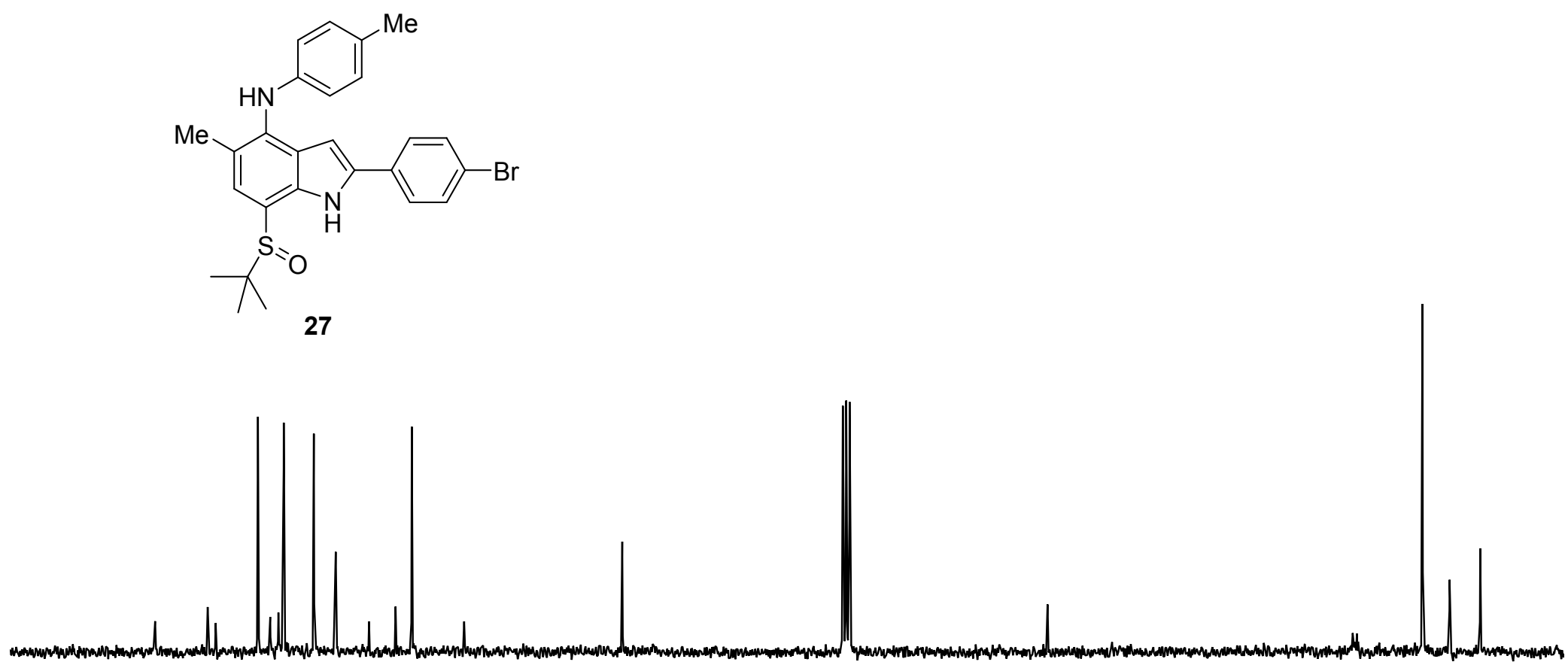

$\begin{array}{llllllllllllll}150 & 140 & 130 & 120 & 110 & 100 & 90 \underset{\mathrm{f} 1(\mathrm{ppm})}{80} & 70 & 60 & 50 & 40 & 30 & 20 & 1\end{array}$




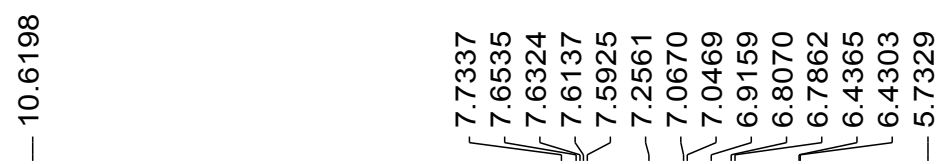

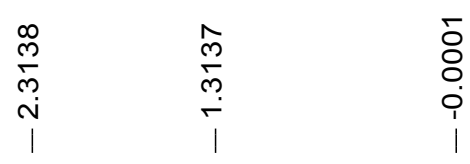
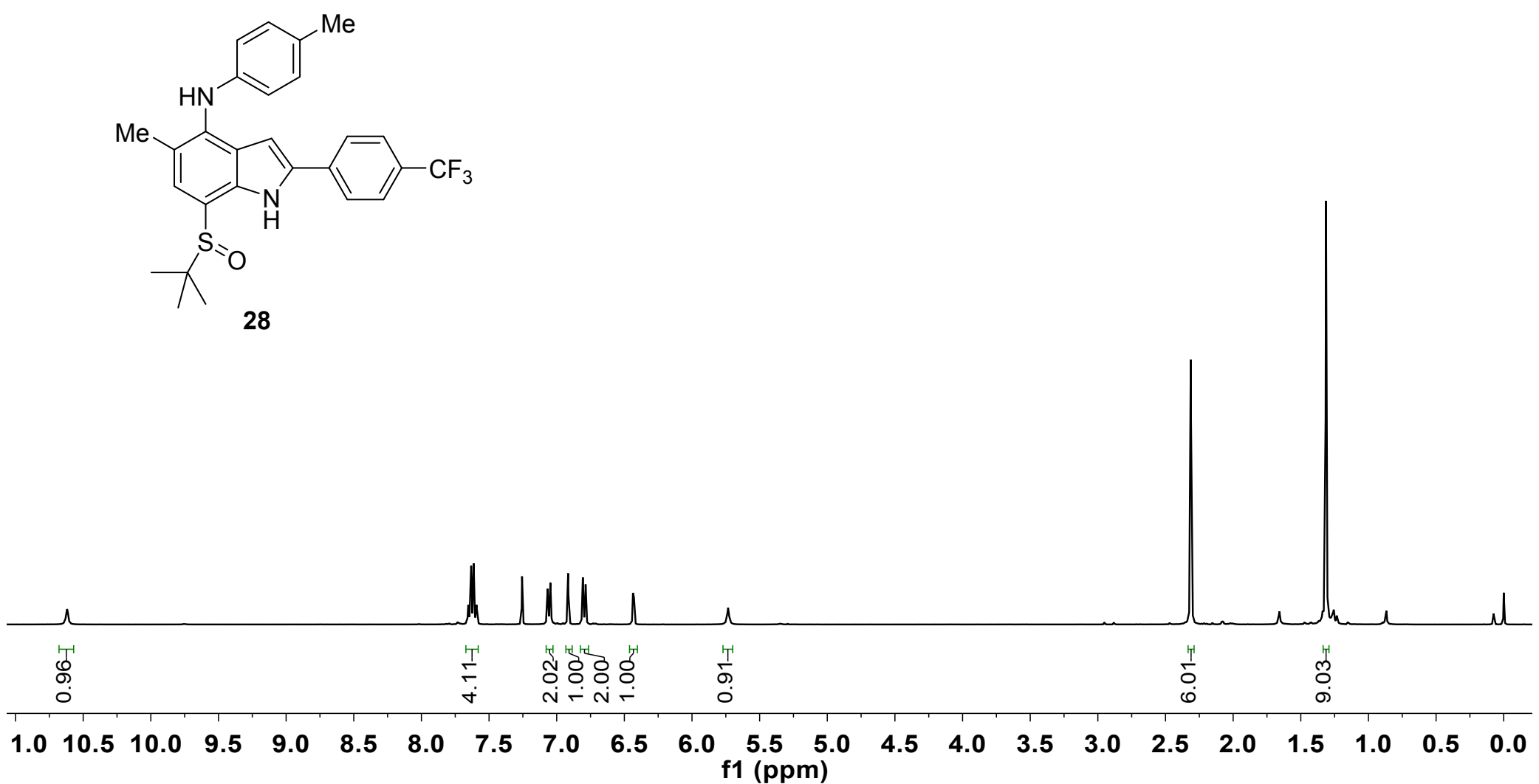

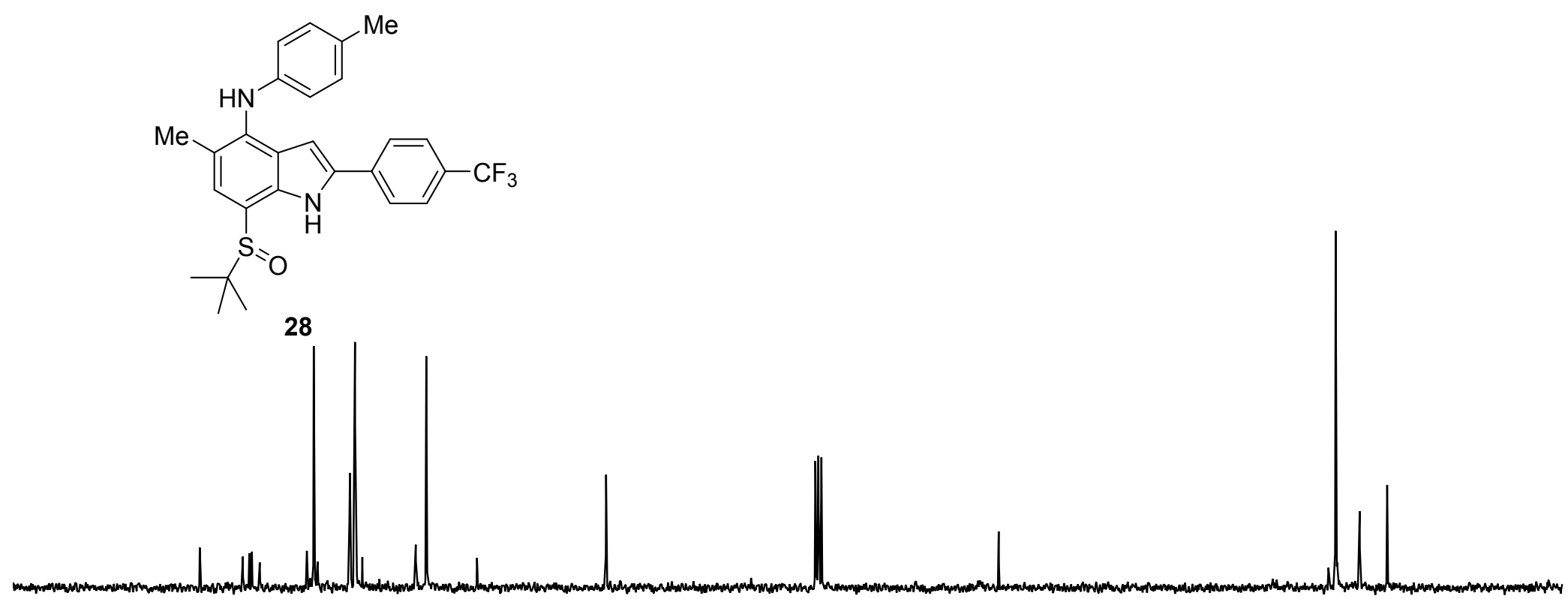


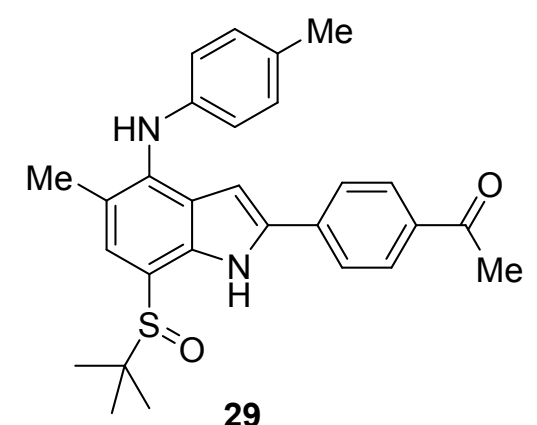

29

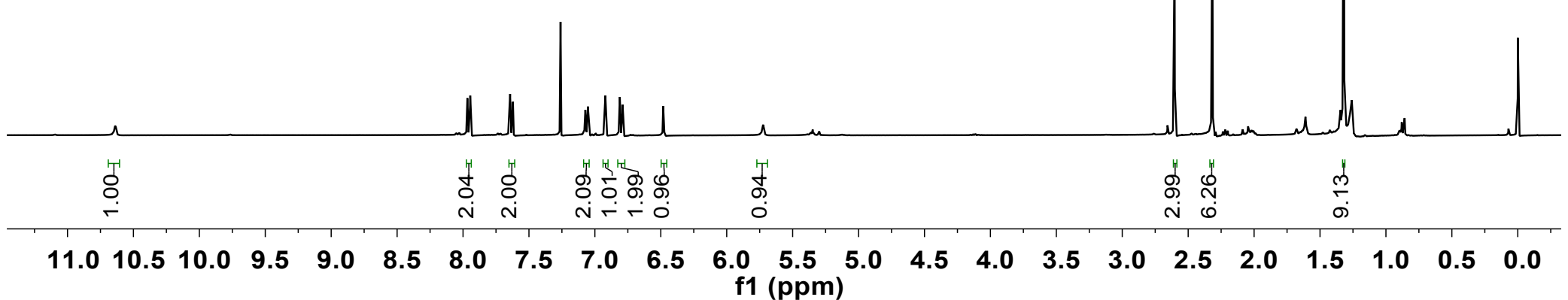



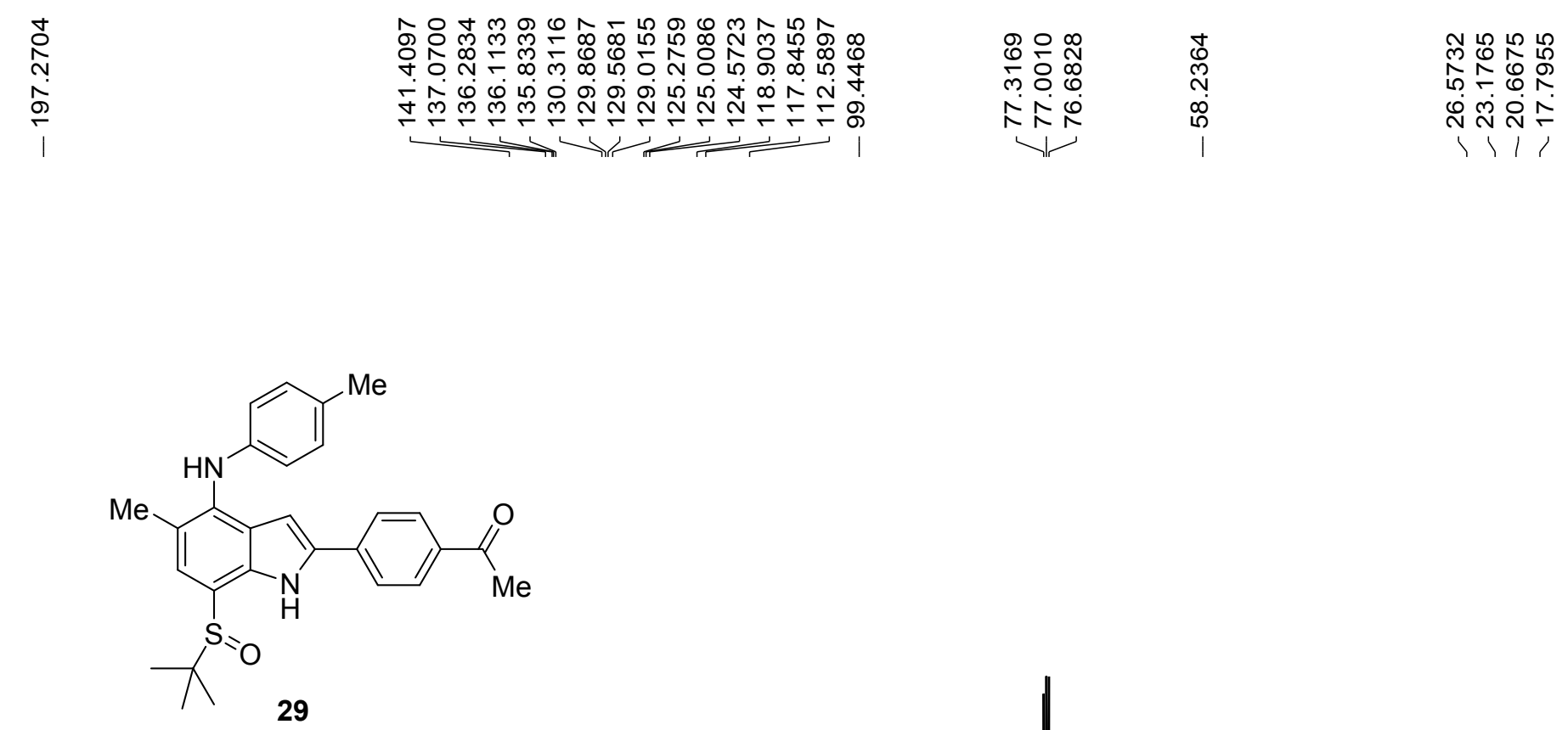

$\begin{array}{lllllllllllllllllllll}210 & 200 & 190 & 180 & 170 & 160 & 150 & 140 & 130 & 120 \begin{array}{c}110 \\ \mathrm{f} 1(\mathrm{ppm})\end{array} & 90 & 80 & 70 & 60 & 50 & 40 & 30 & 20 & 10\end{array}$ 

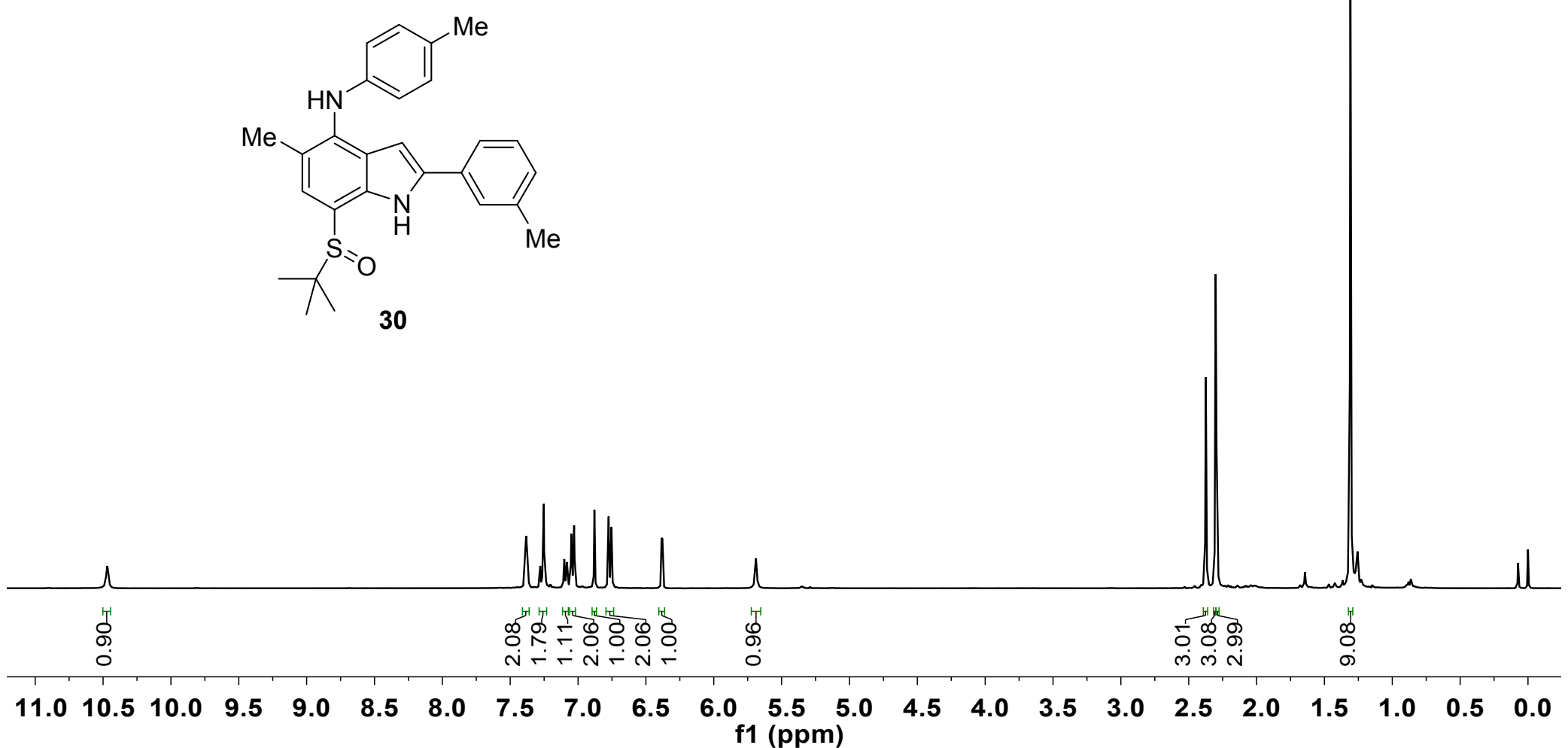

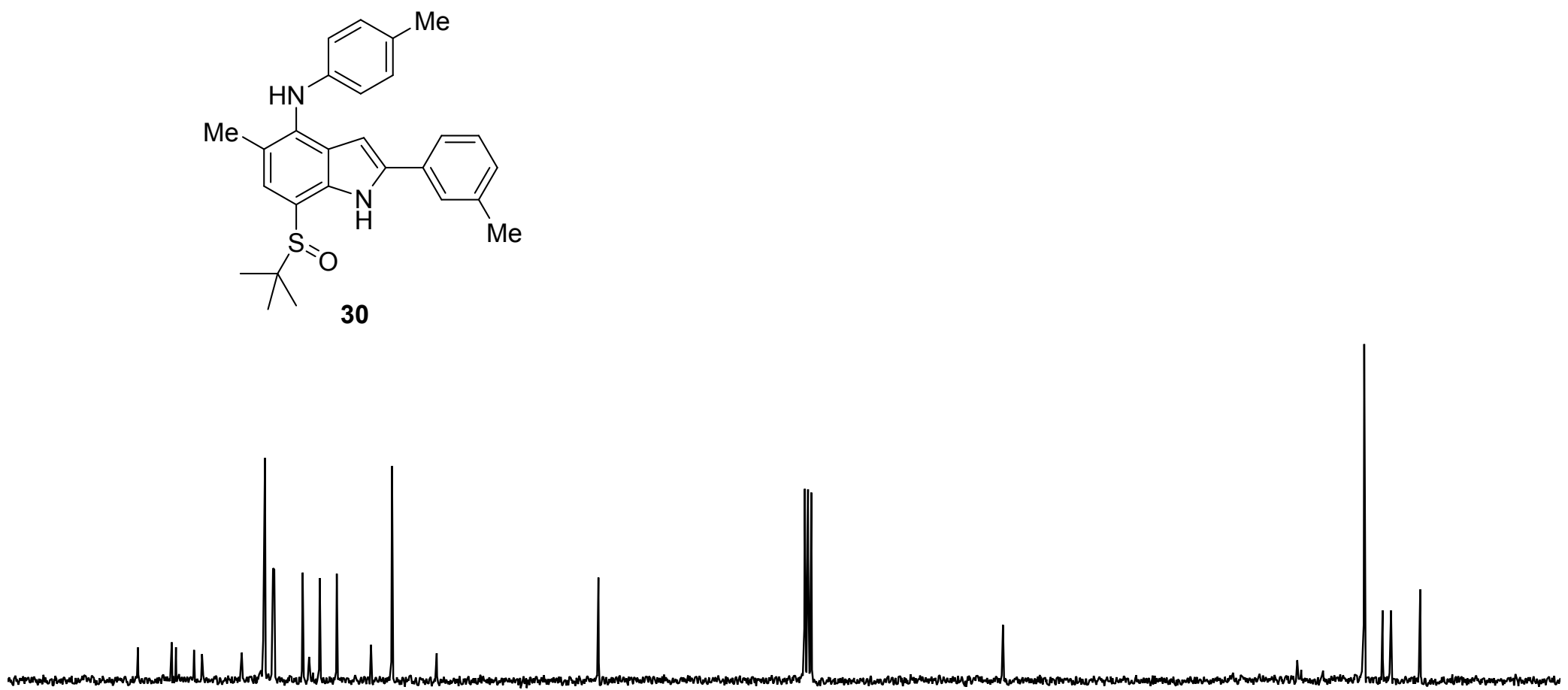

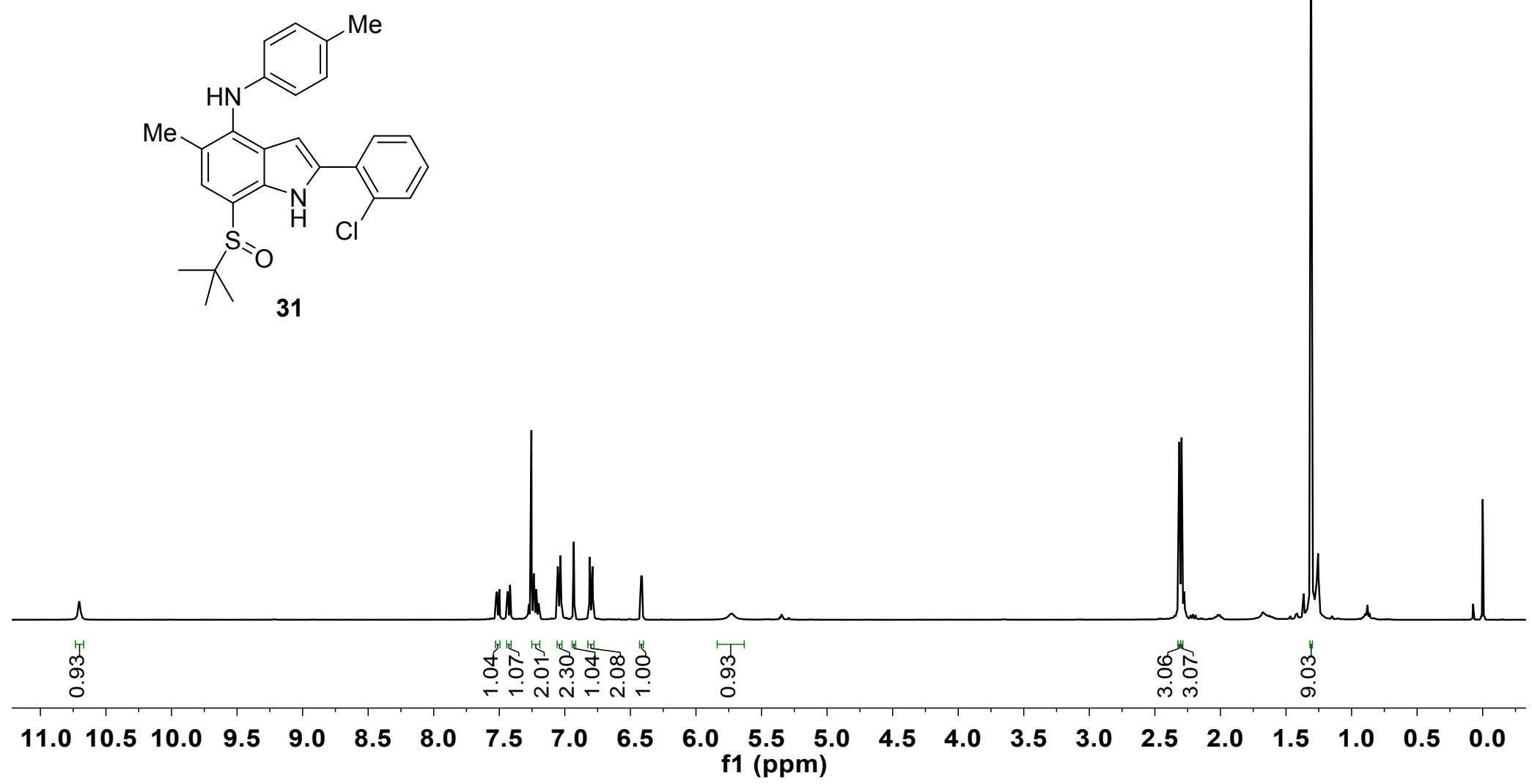

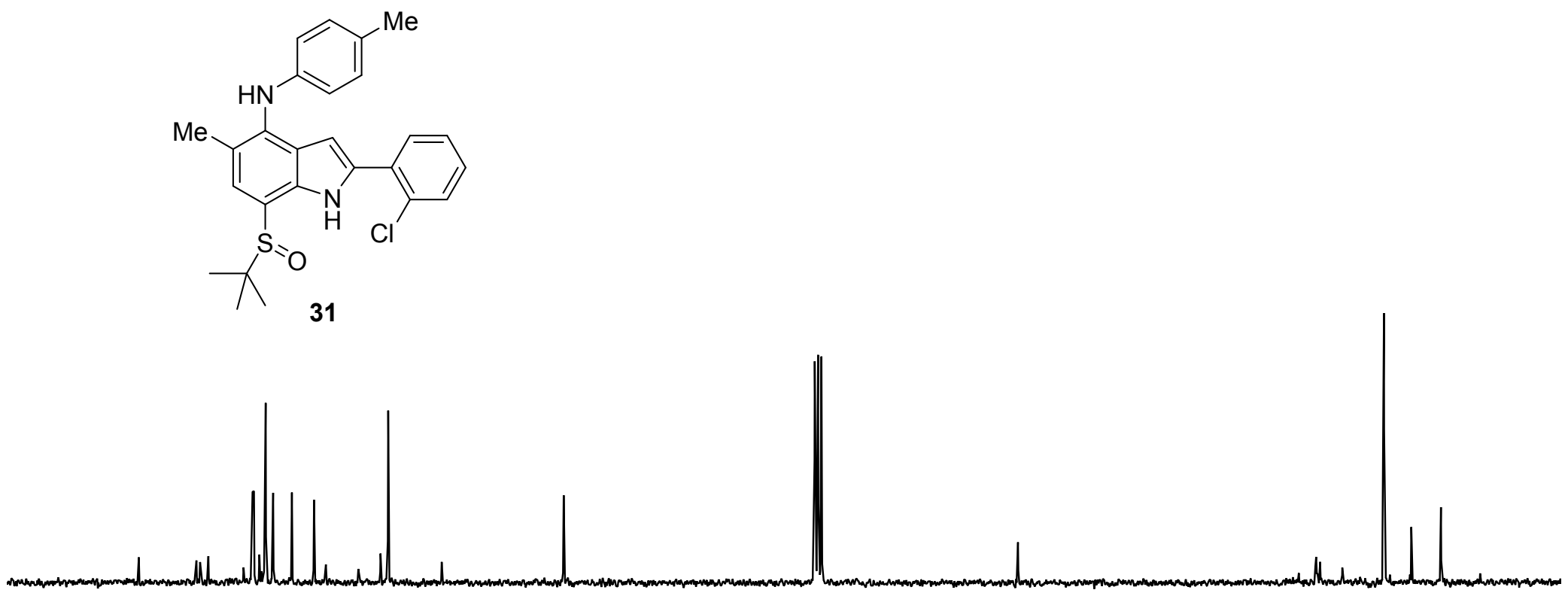
f1 (ppm) 

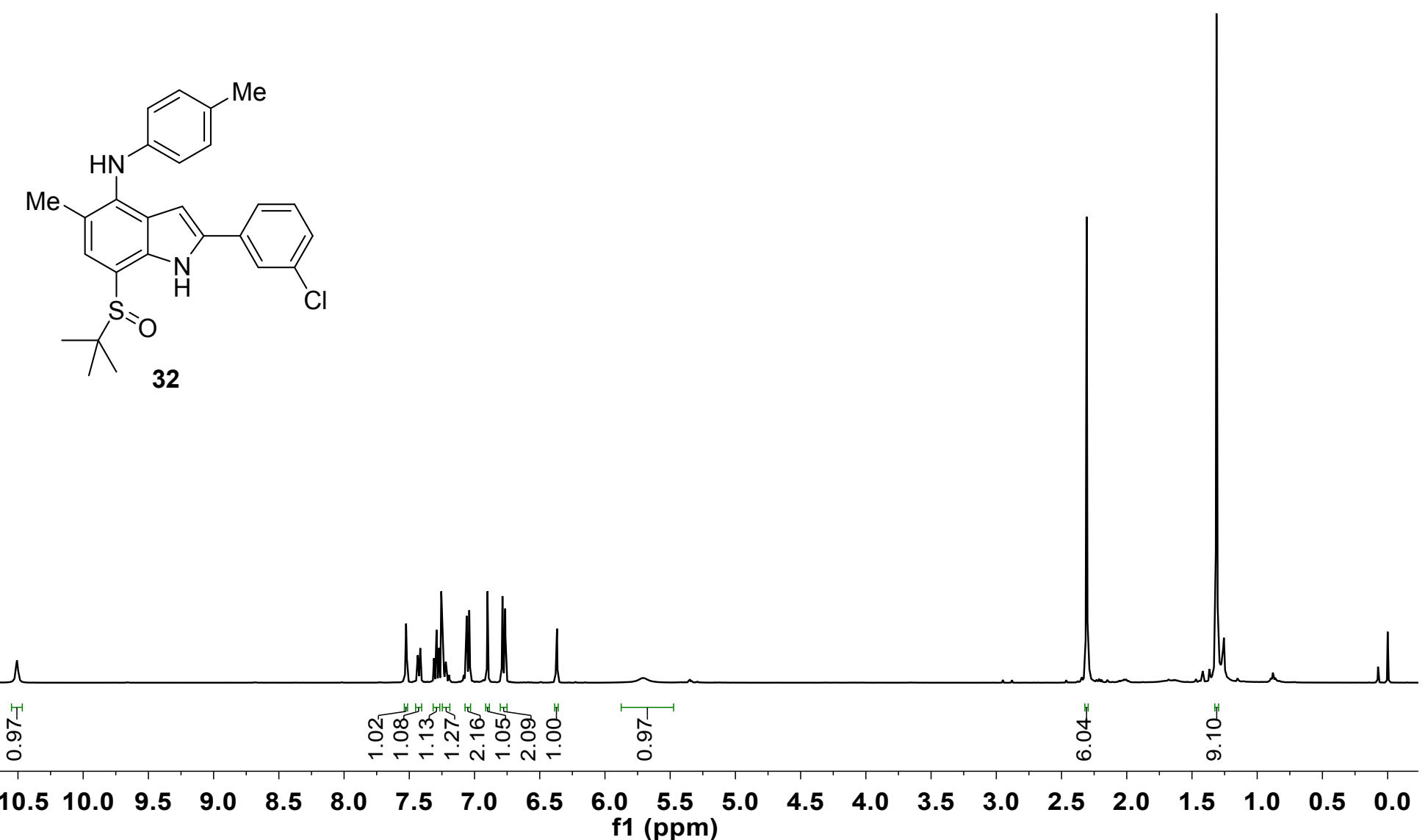

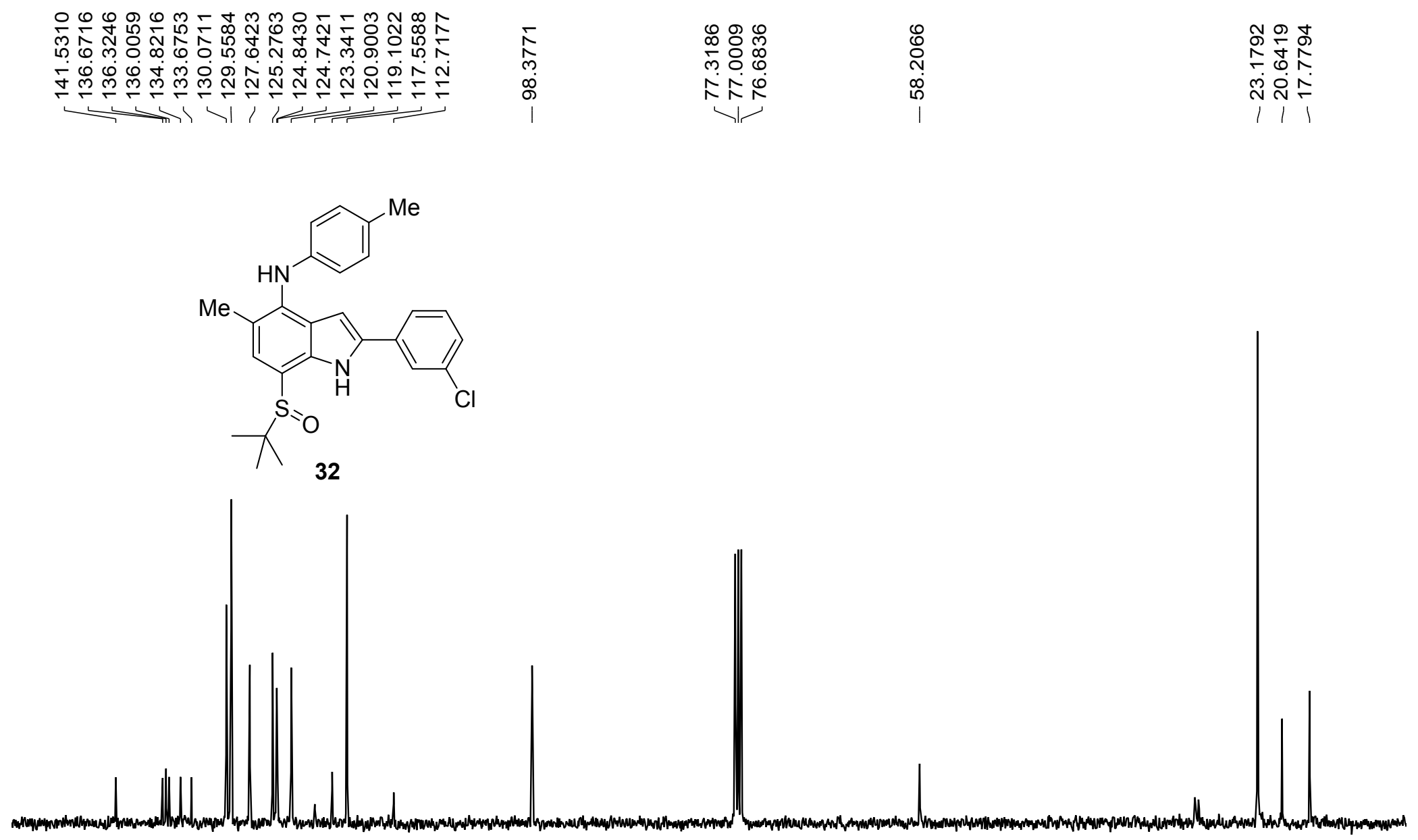

$15014514013513012512011511010510095 \quad \begin{gathered}90 \\ 85 \\ \mathrm{f} 1(\mathrm{ppm})\end{gathered}$ 

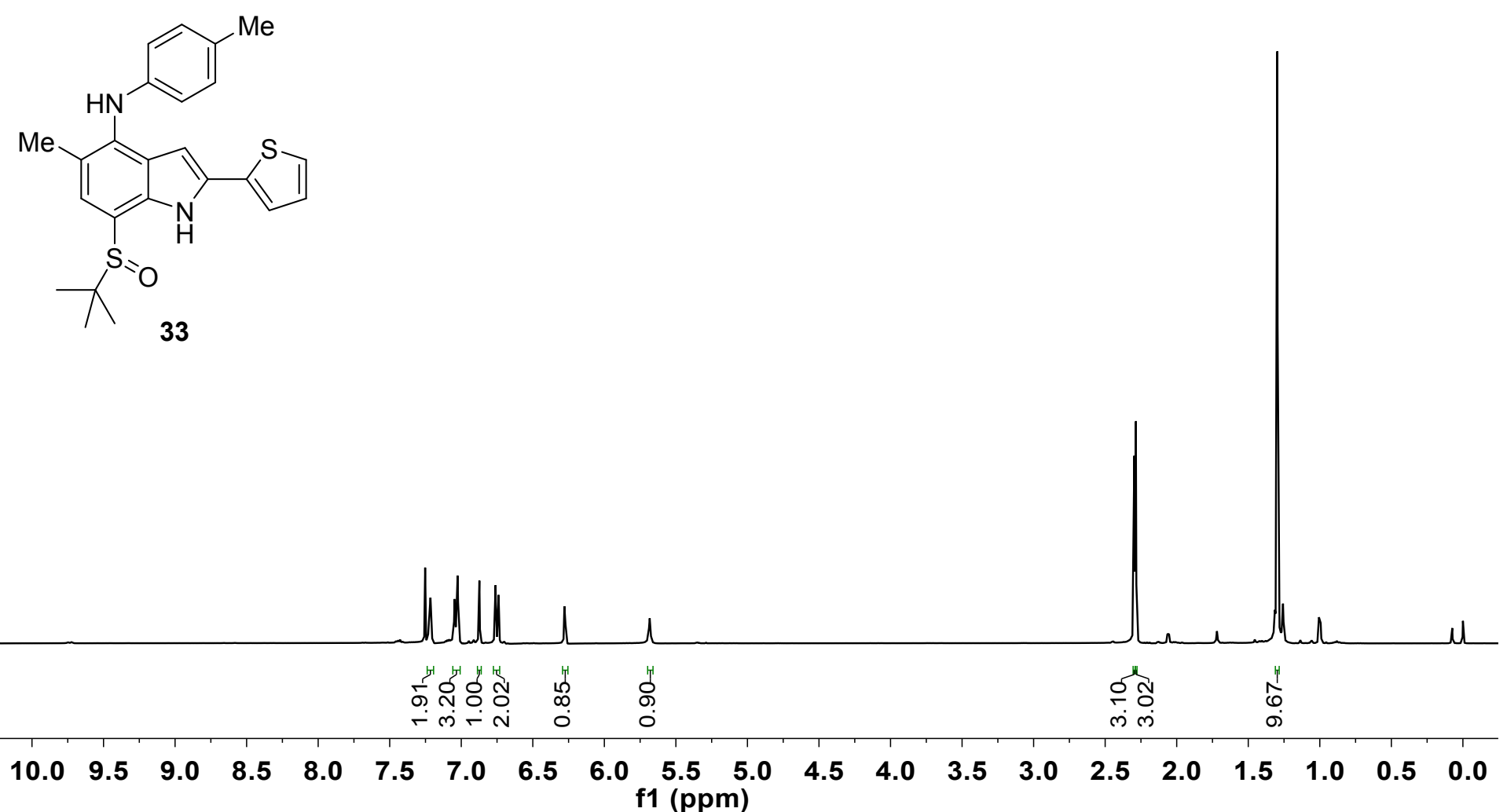

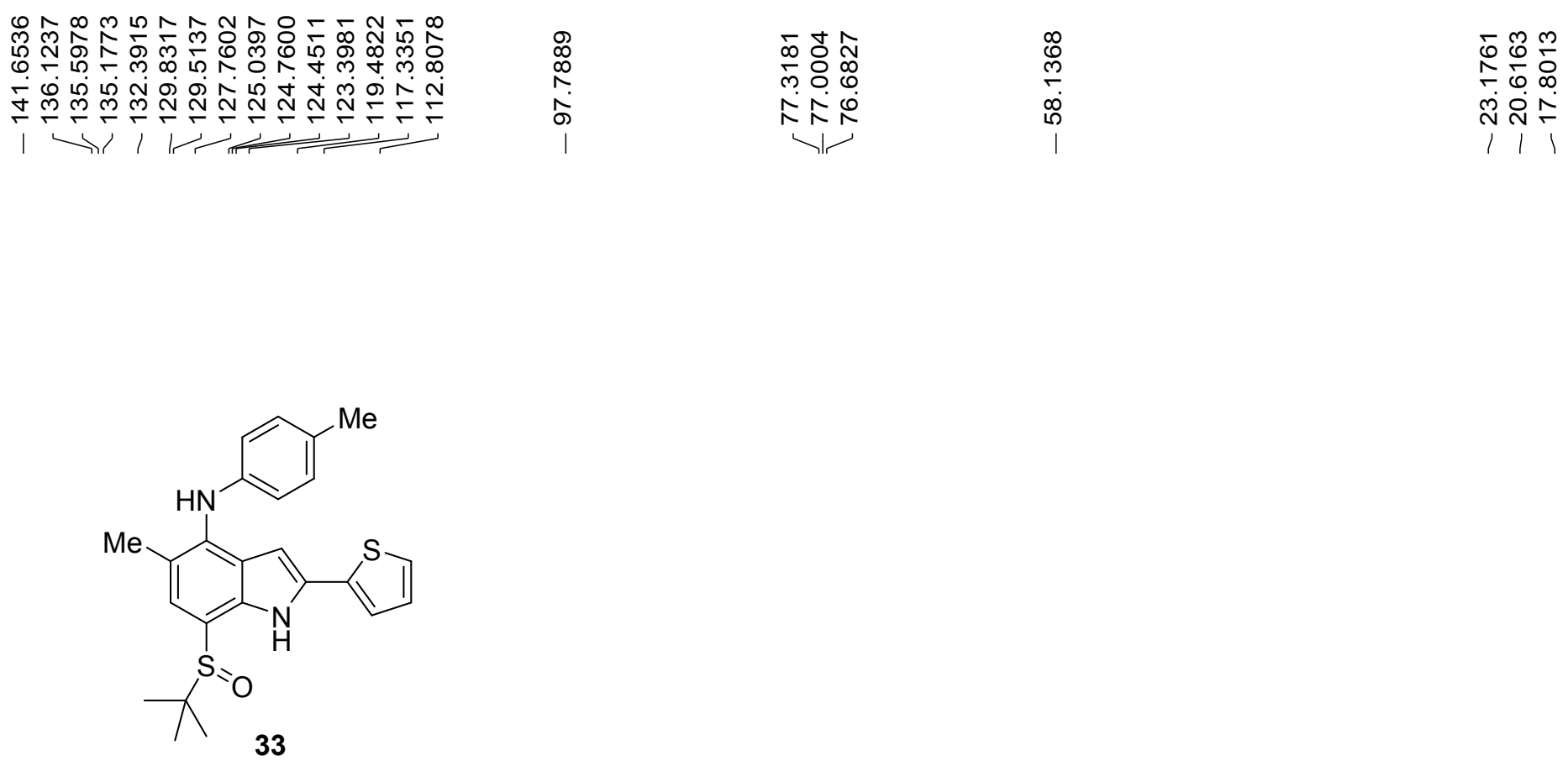

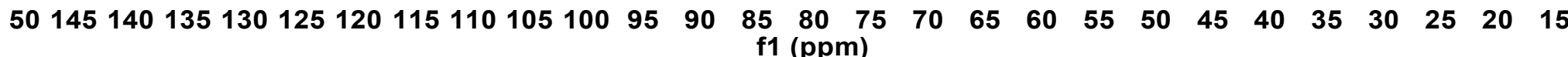



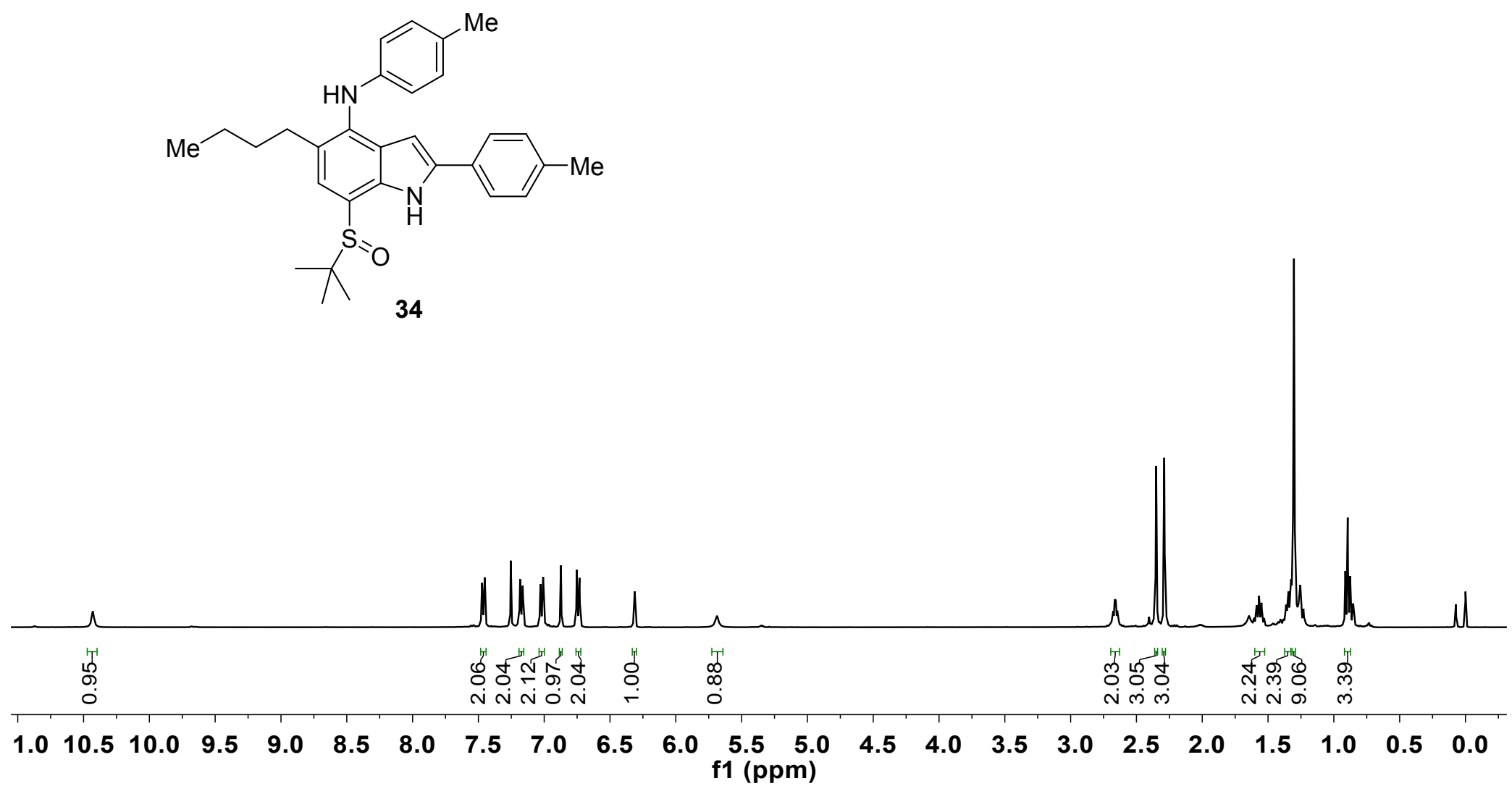


\begin{tabular}{|c|c|c|c|c|c|}
\hline 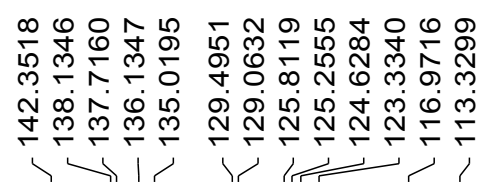 & 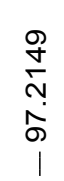 & 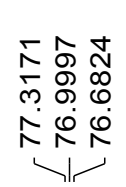 & $\begin{array}{c}0 \\
\frac{0}{N} \\
\frac{0}{\infty} \\
0 \\
1\end{array}$ & 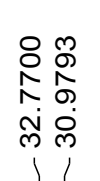 & 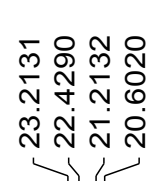 \\
\hline
\end{tabular}

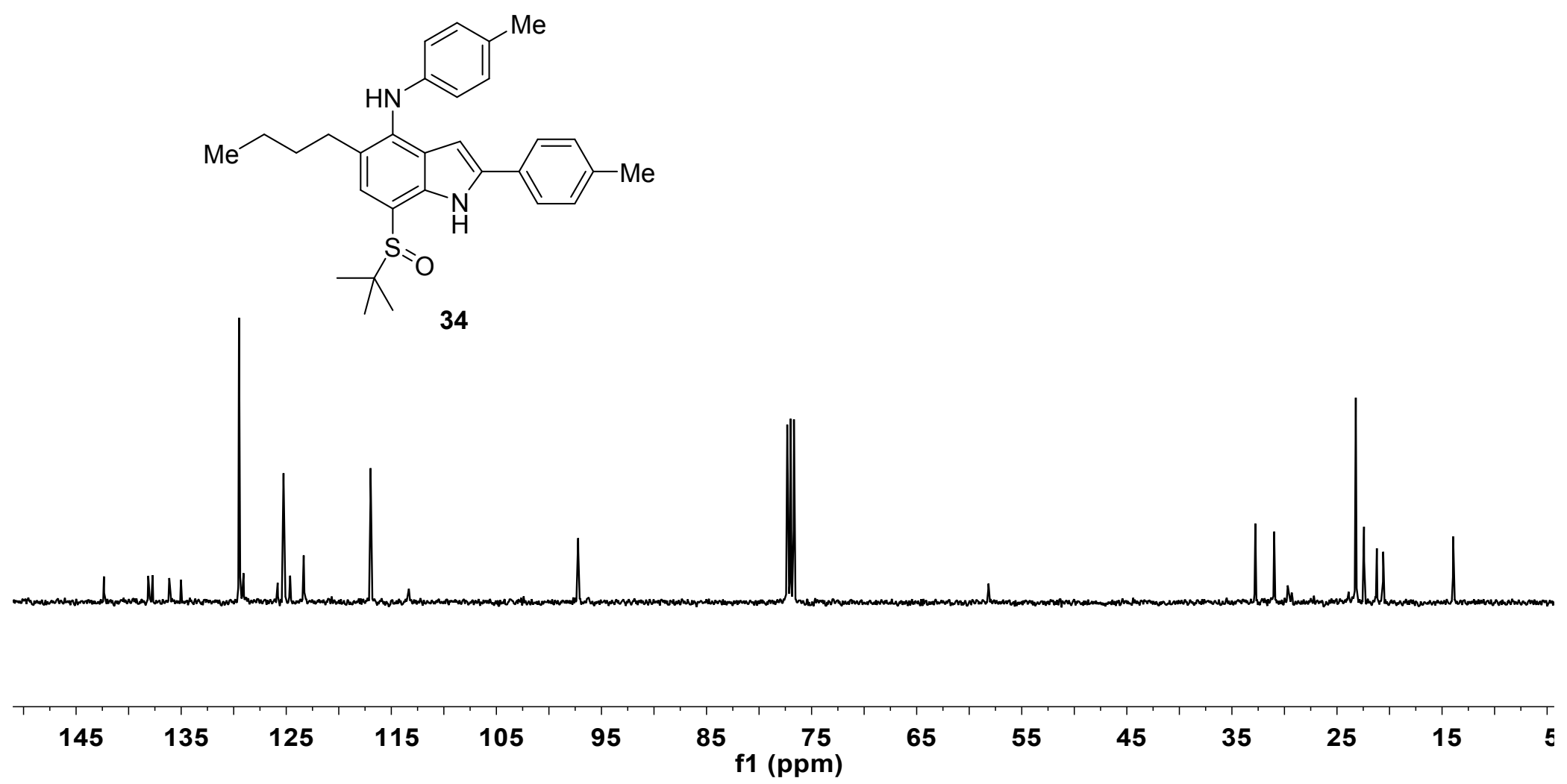



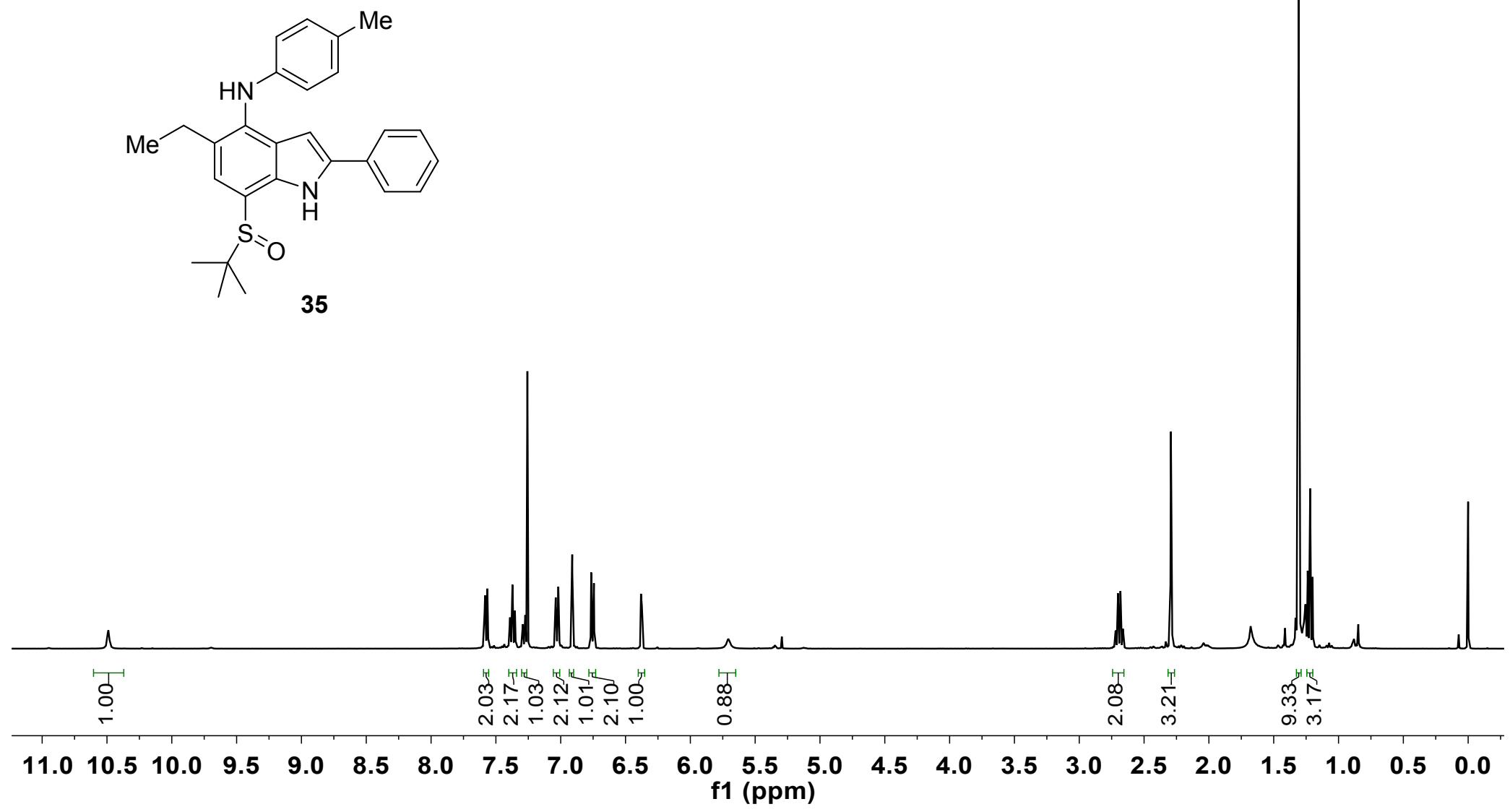

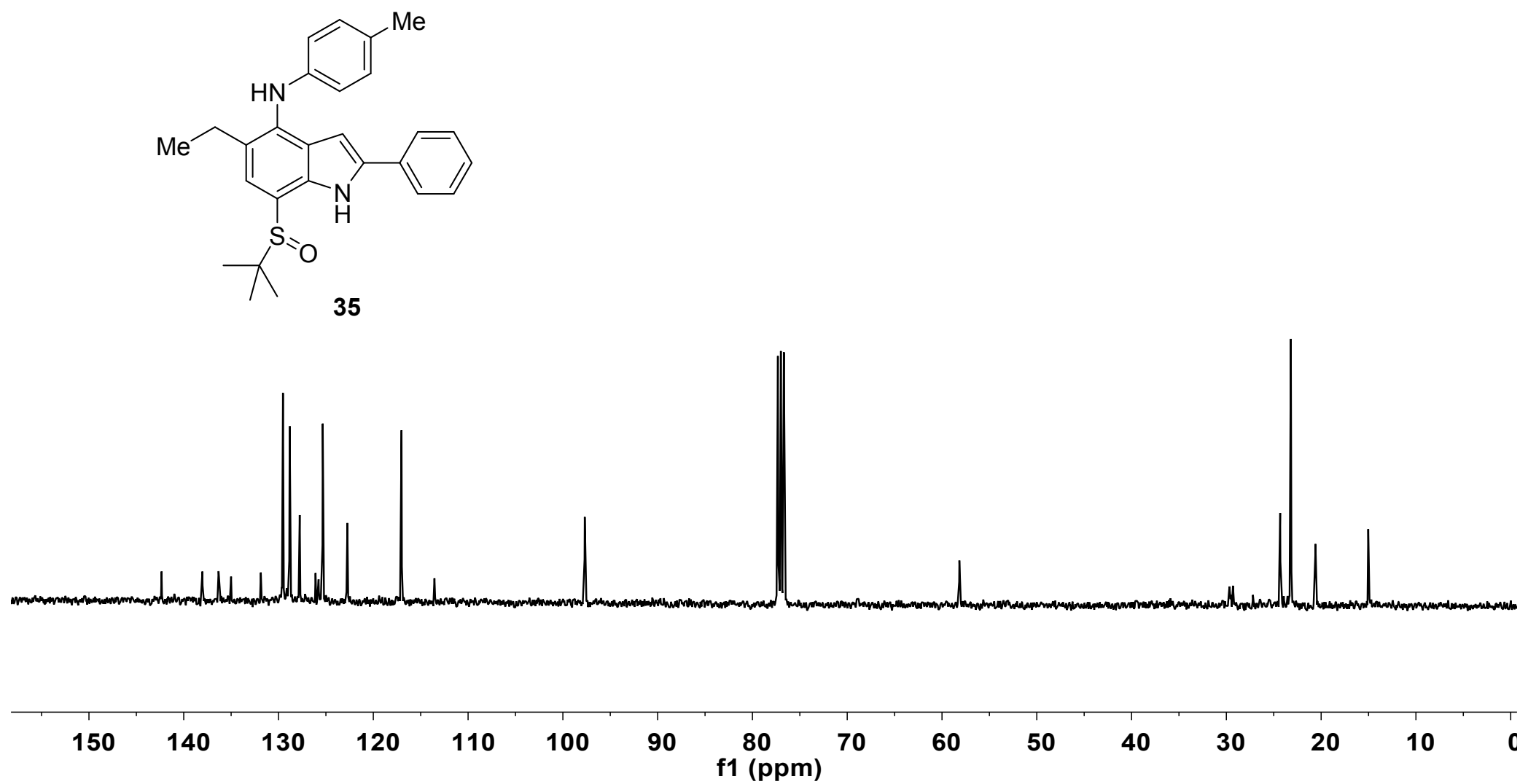


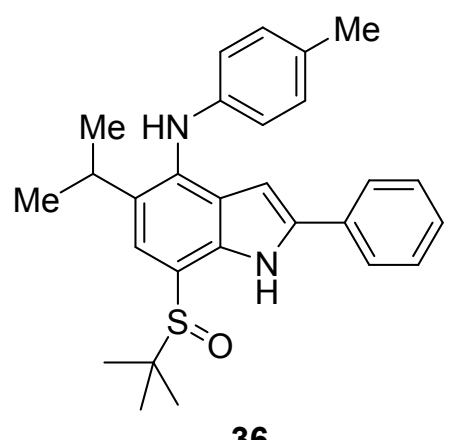

36

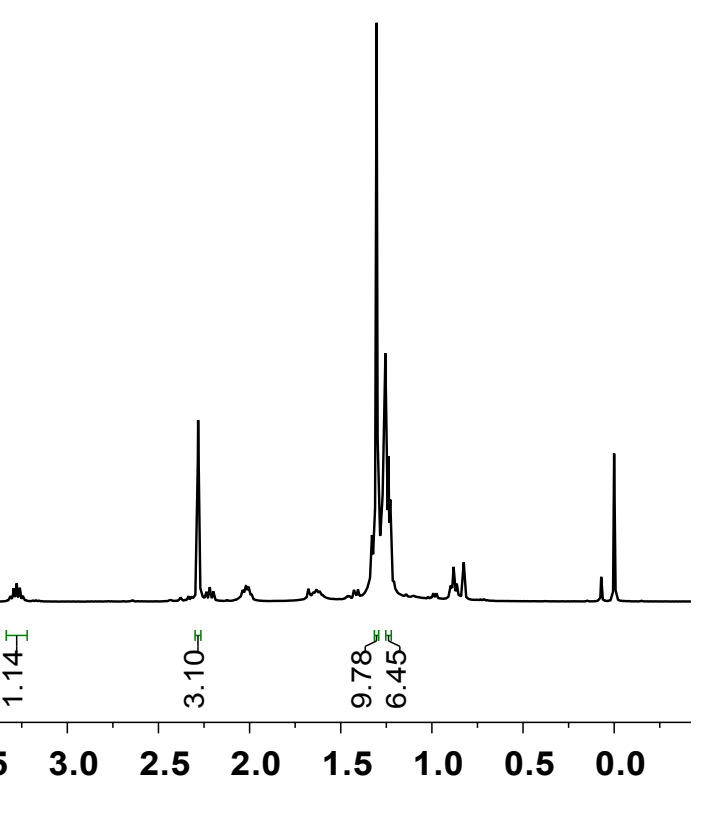




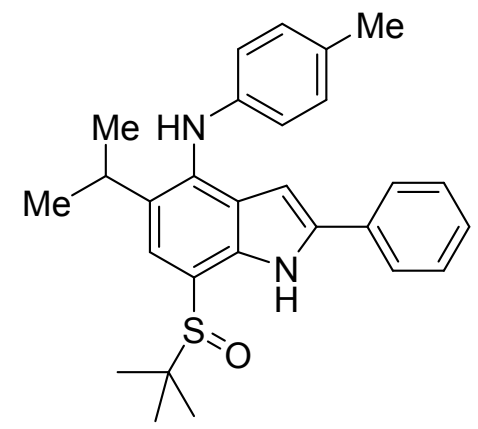

36

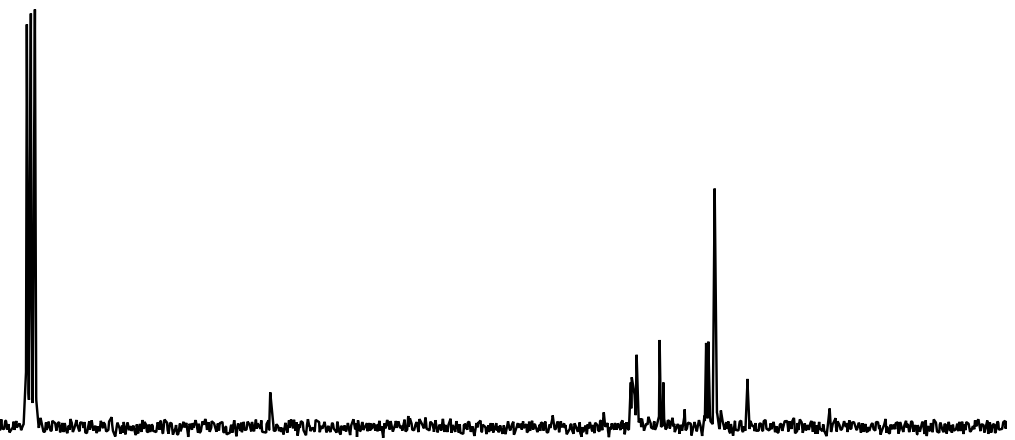
f1 (ppm) 

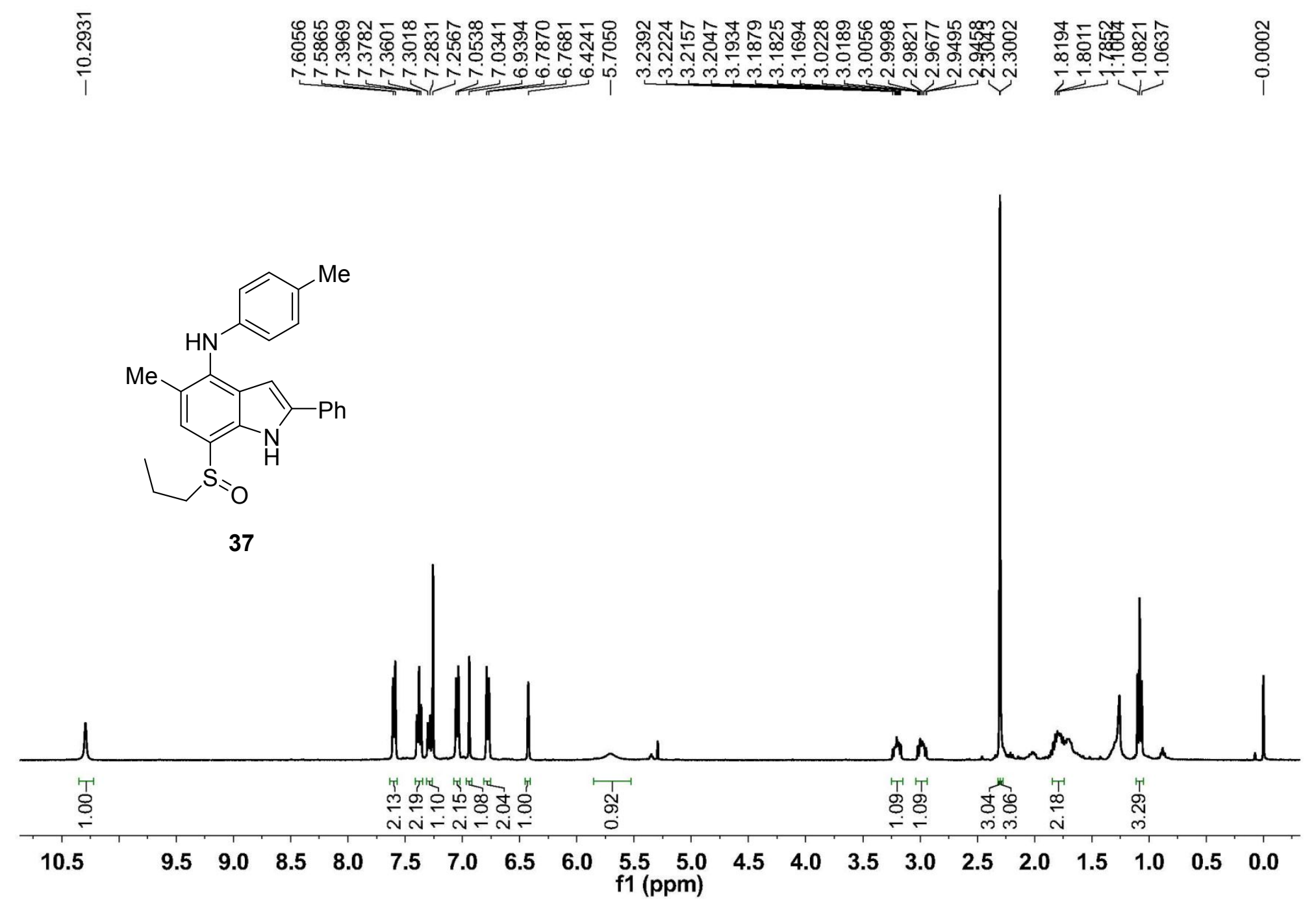


\begin{tabular}{|c|c|c|c|c|}
\hline 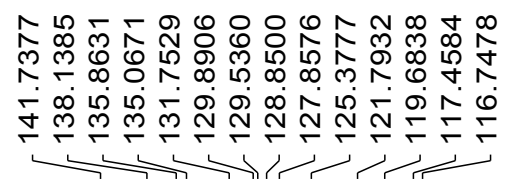 & $\begin{array}{l}\text { O } \\
\frac{\infty}{0} \\
\infty \\
\infty \\
\infty \\
\end{array}$ & 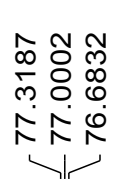 & $\begin{array}{c}\stackrel{m}{m} \\
\stackrel{N}{N} \\
\infty \\
\infty \\
\infty \\
1\end{array}$ & 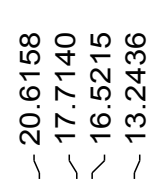 \\
\hline
\end{tabular}
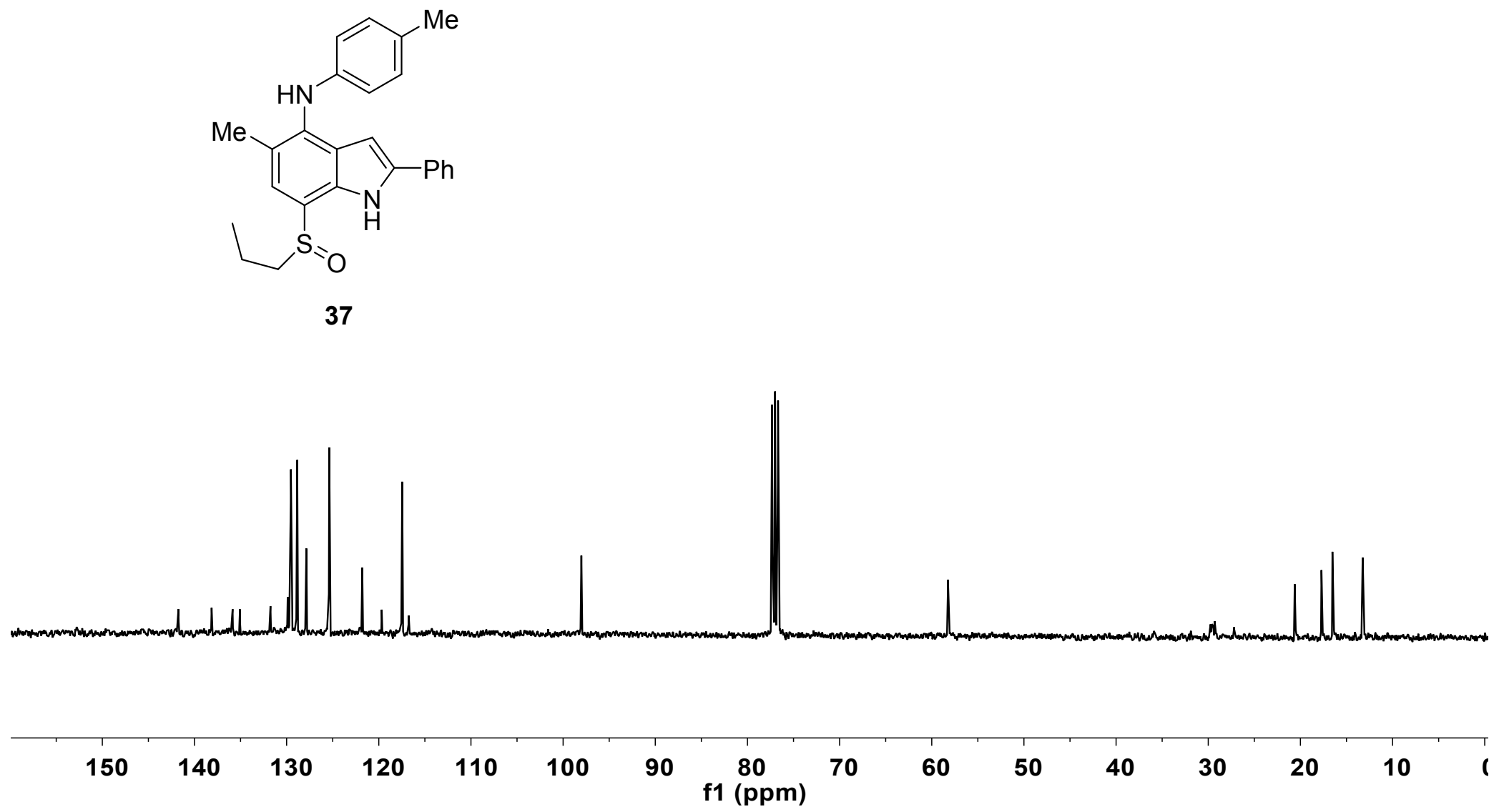

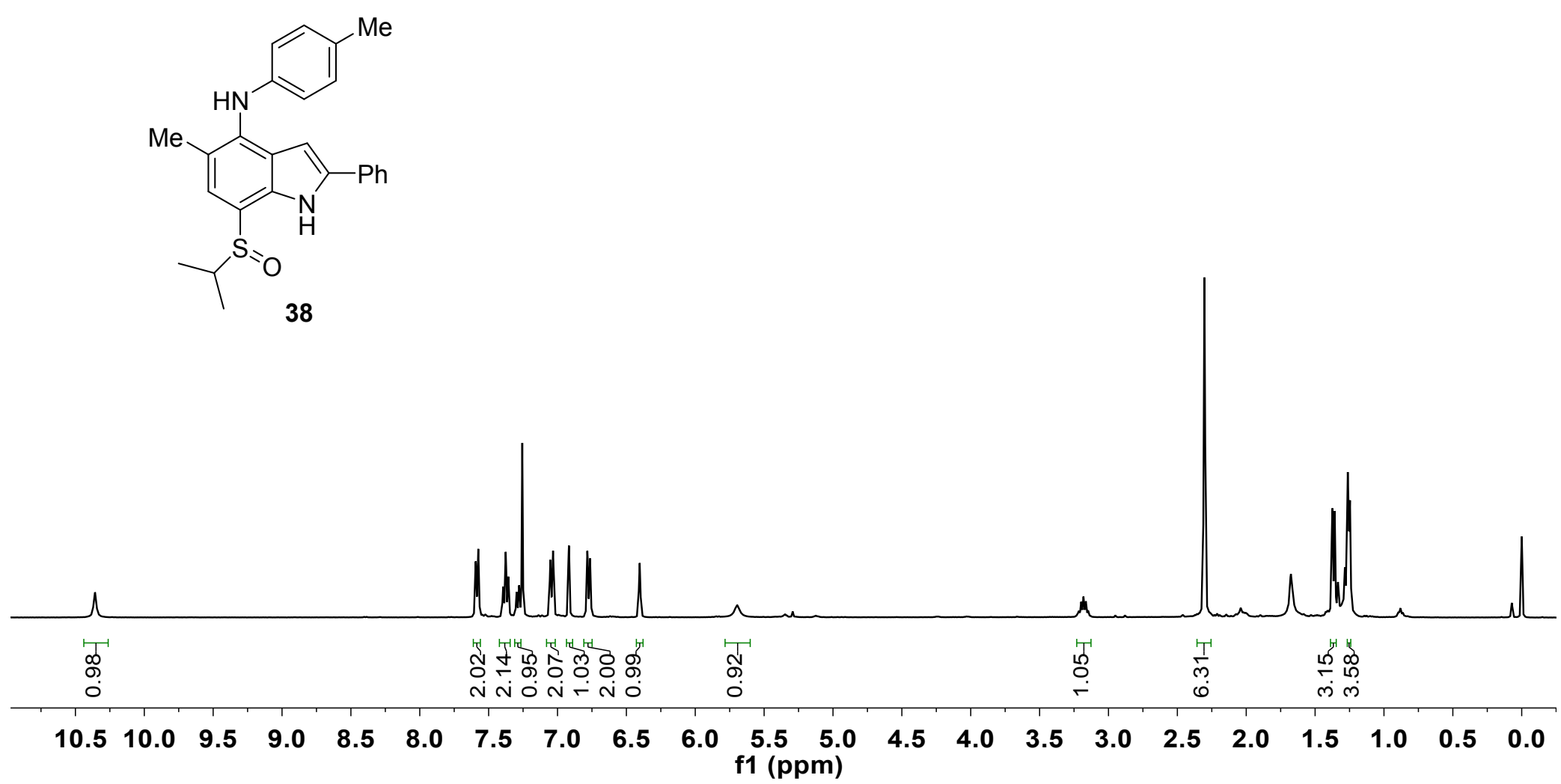


\begin{tabular}{|c|c|c|c|}
\hline 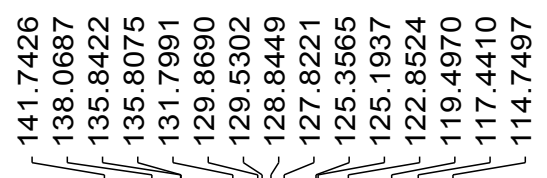 & 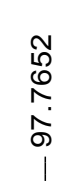 & 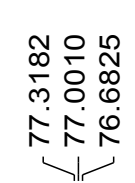 & $\begin{array}{l}\overline{0} \\
0 \\
0 \\
0 \\
0 \\
1\end{array}$ \\
\hline
\end{tabular}
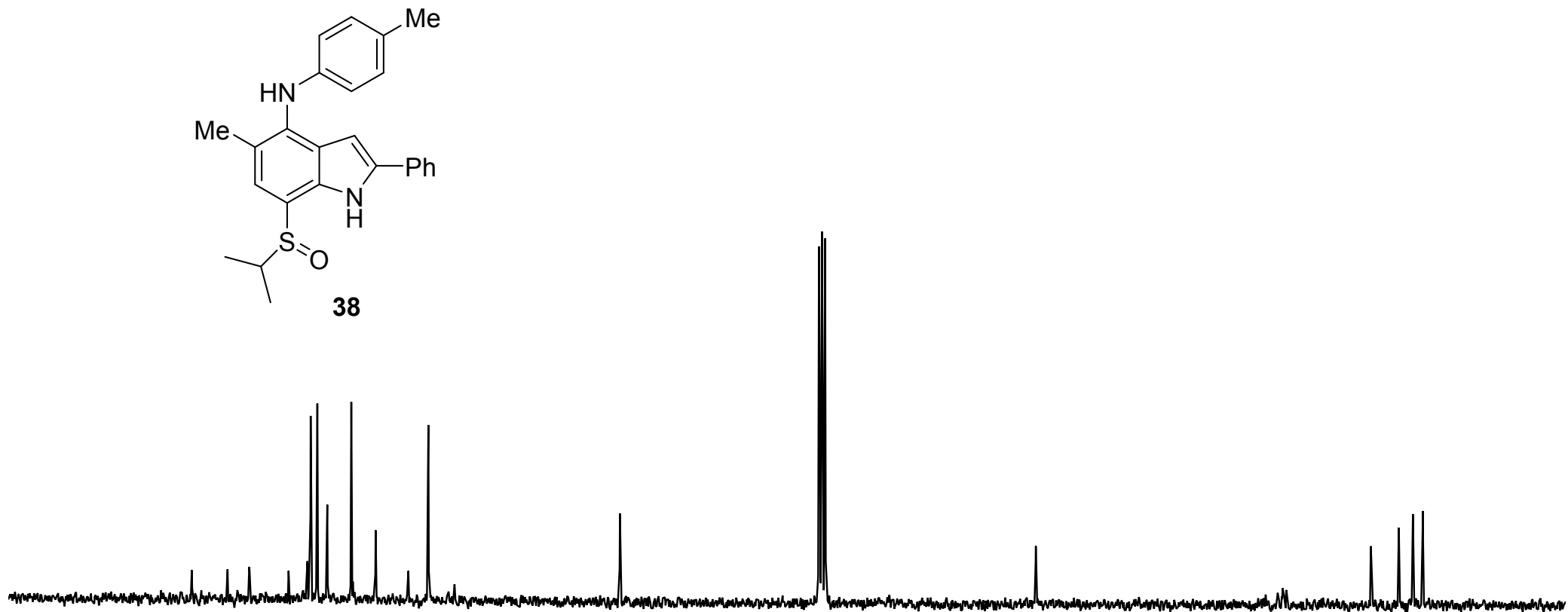

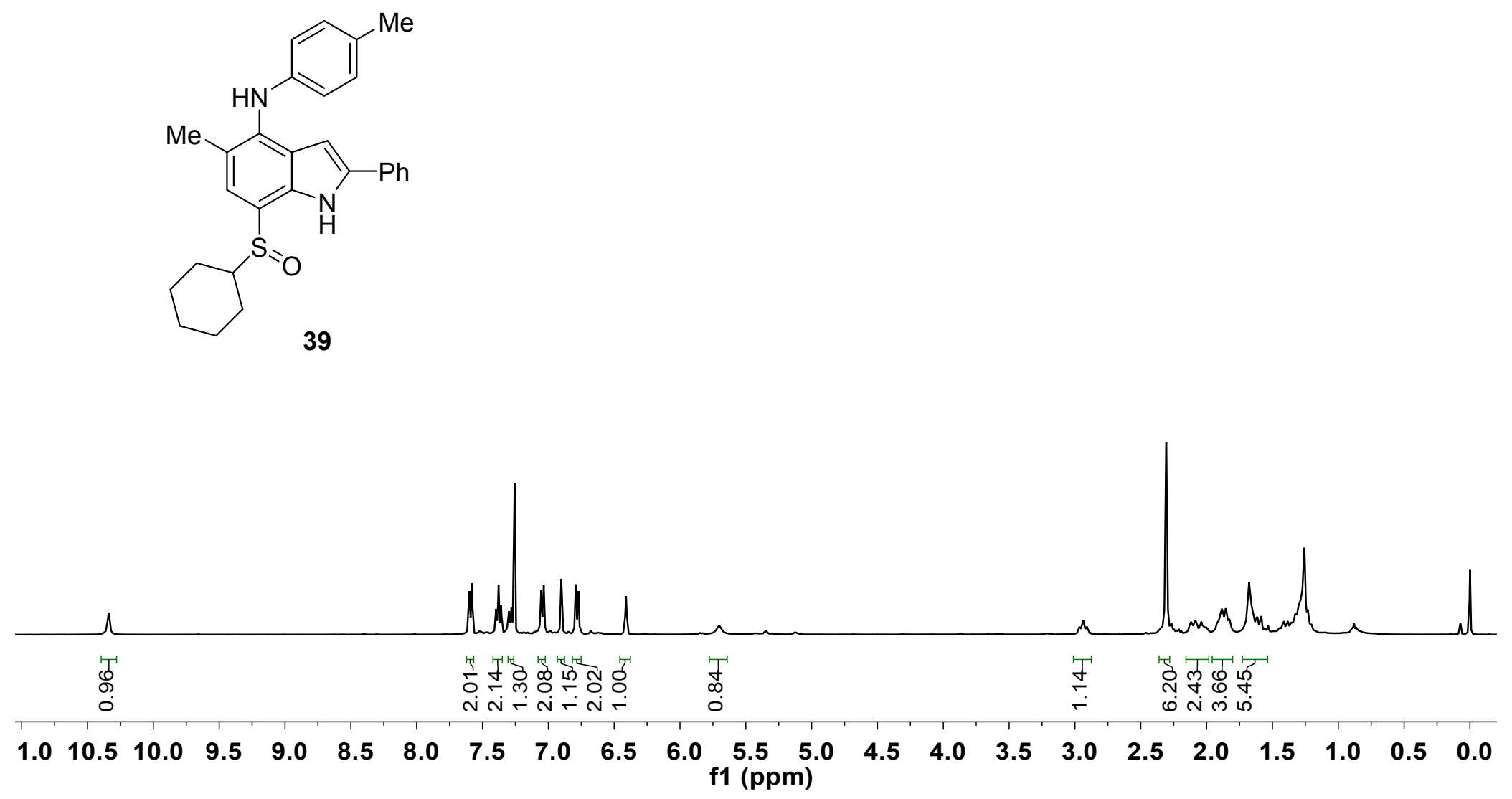

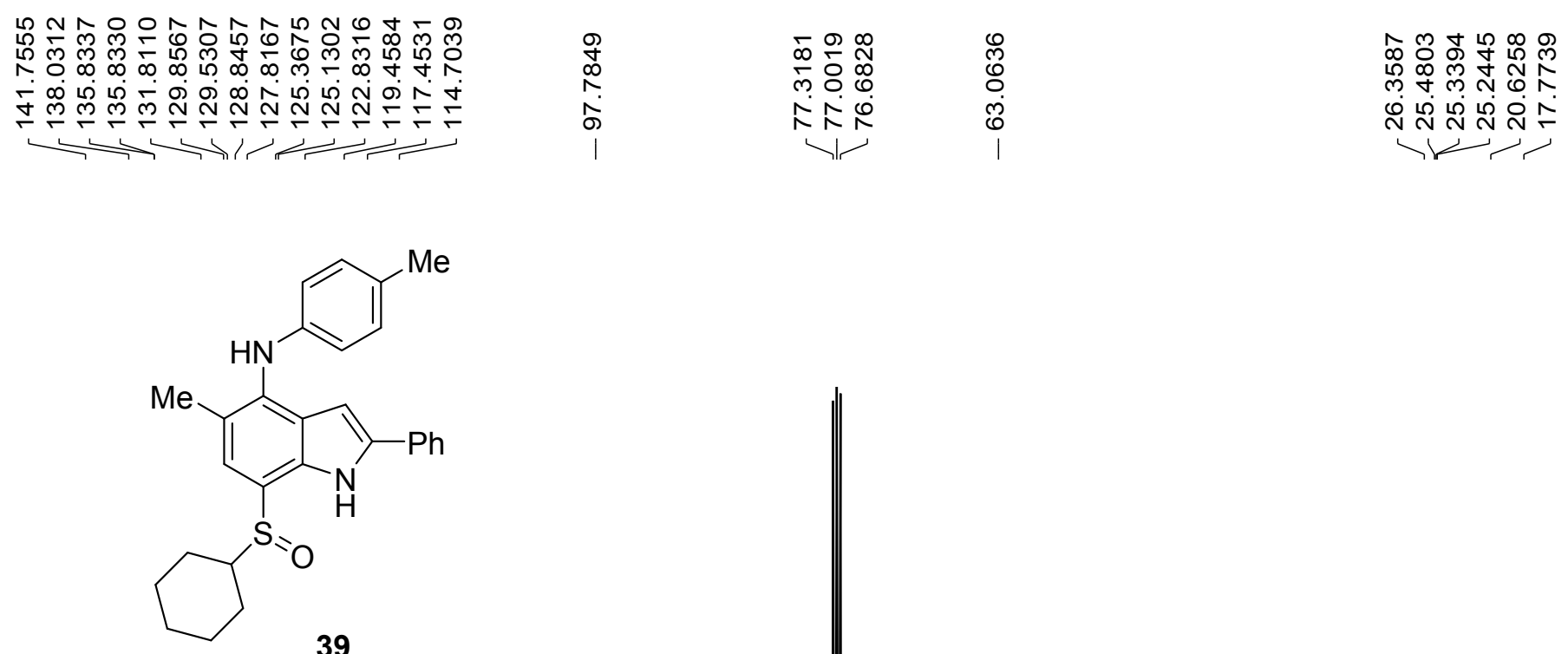

39

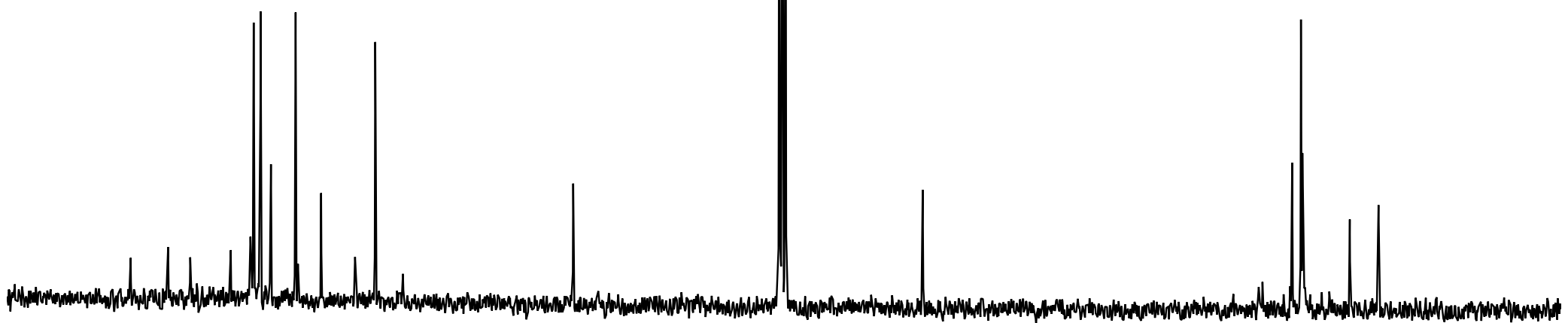

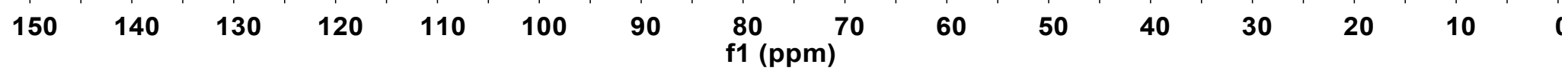




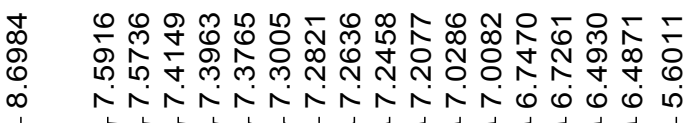

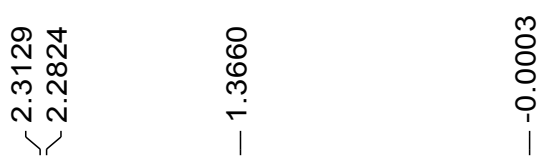

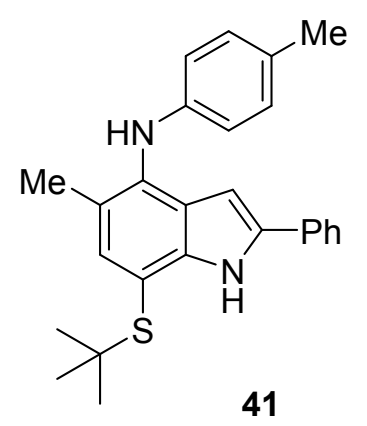

lduld

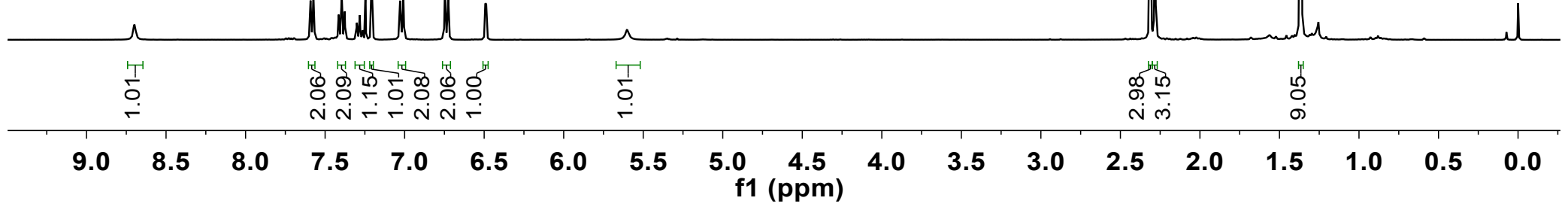




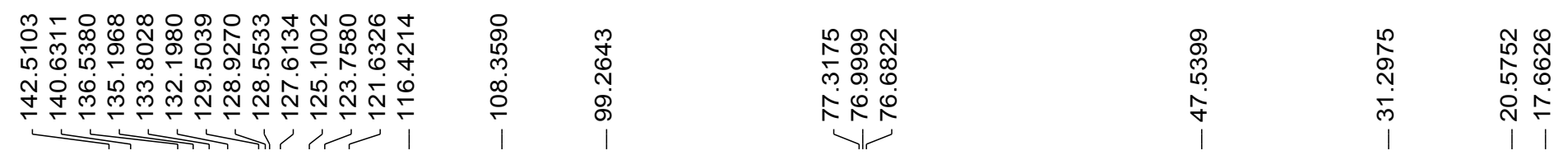
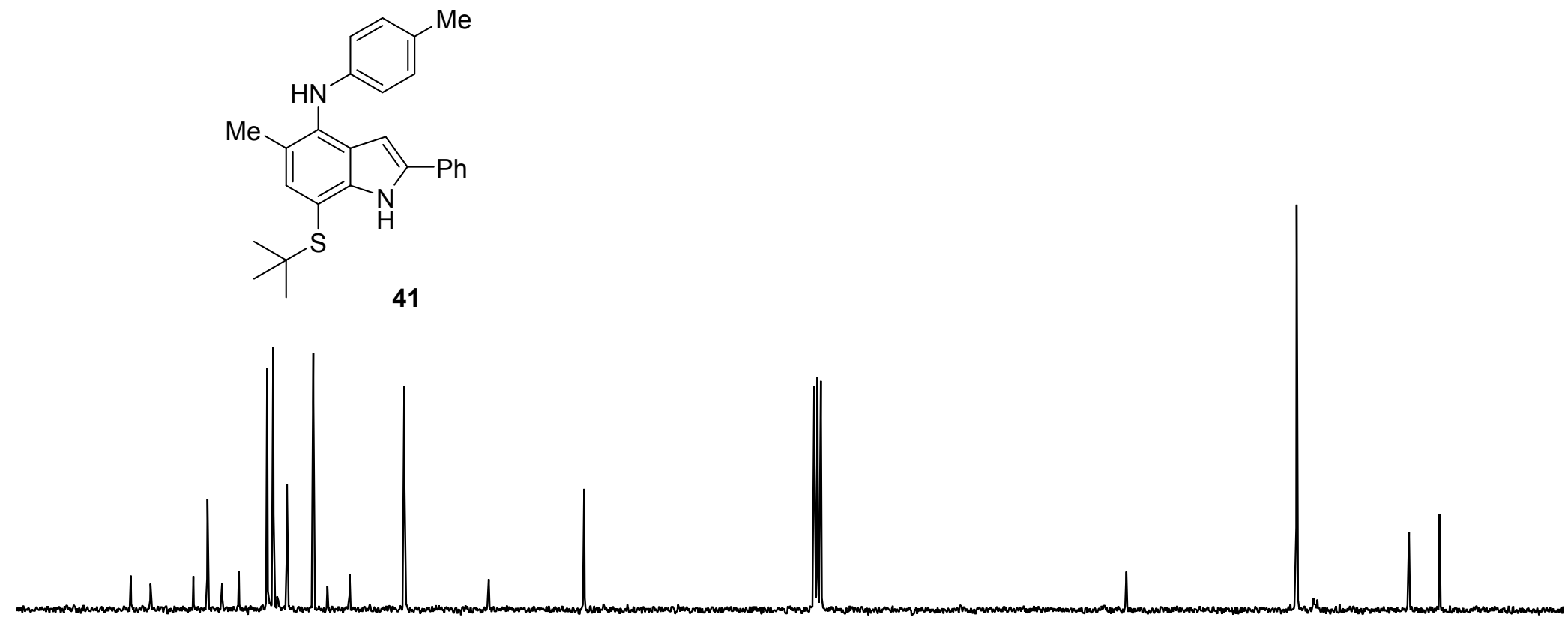

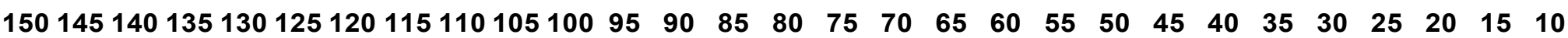
f1 (ppm) 


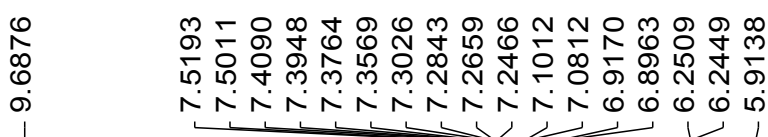
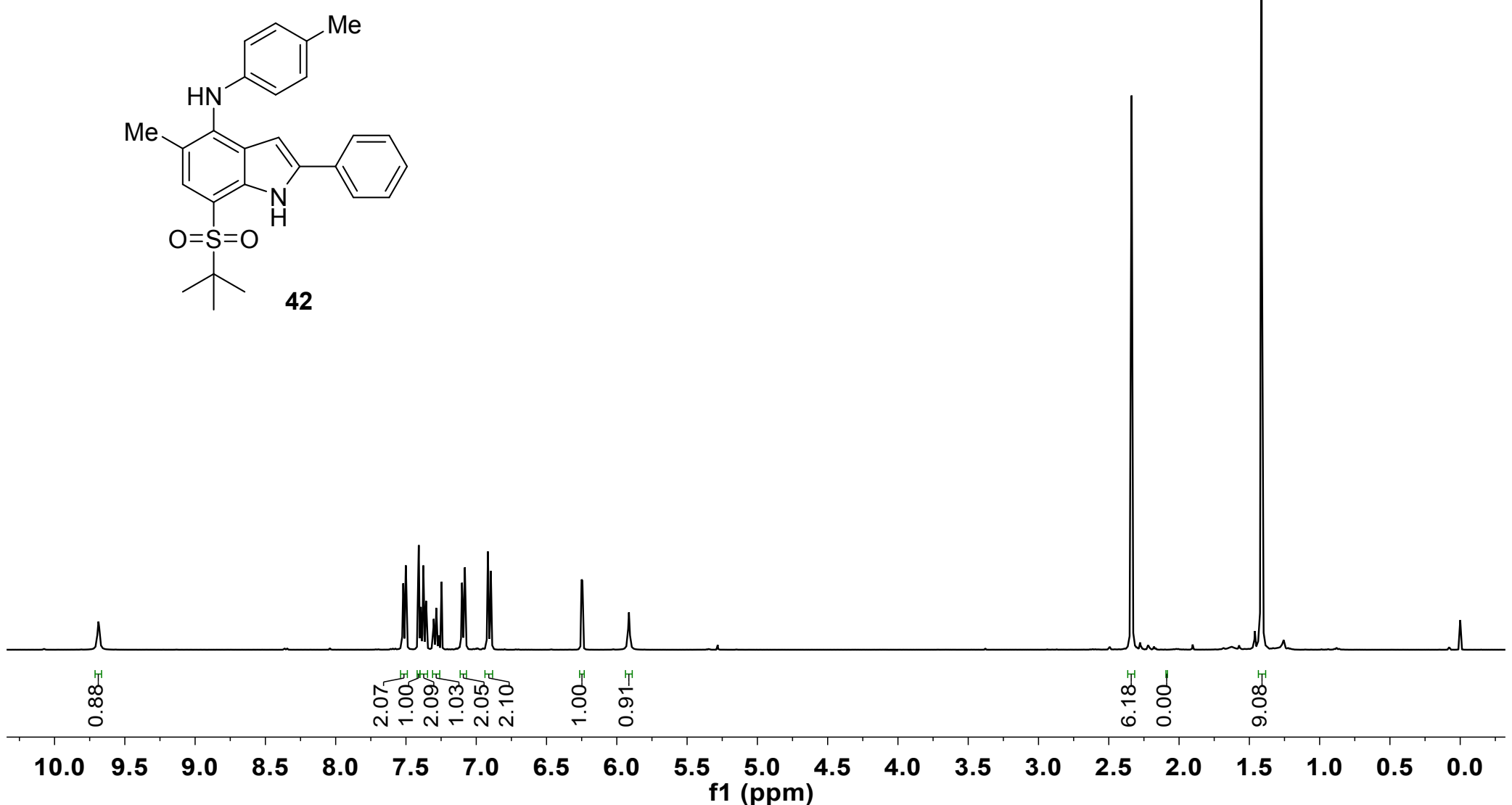

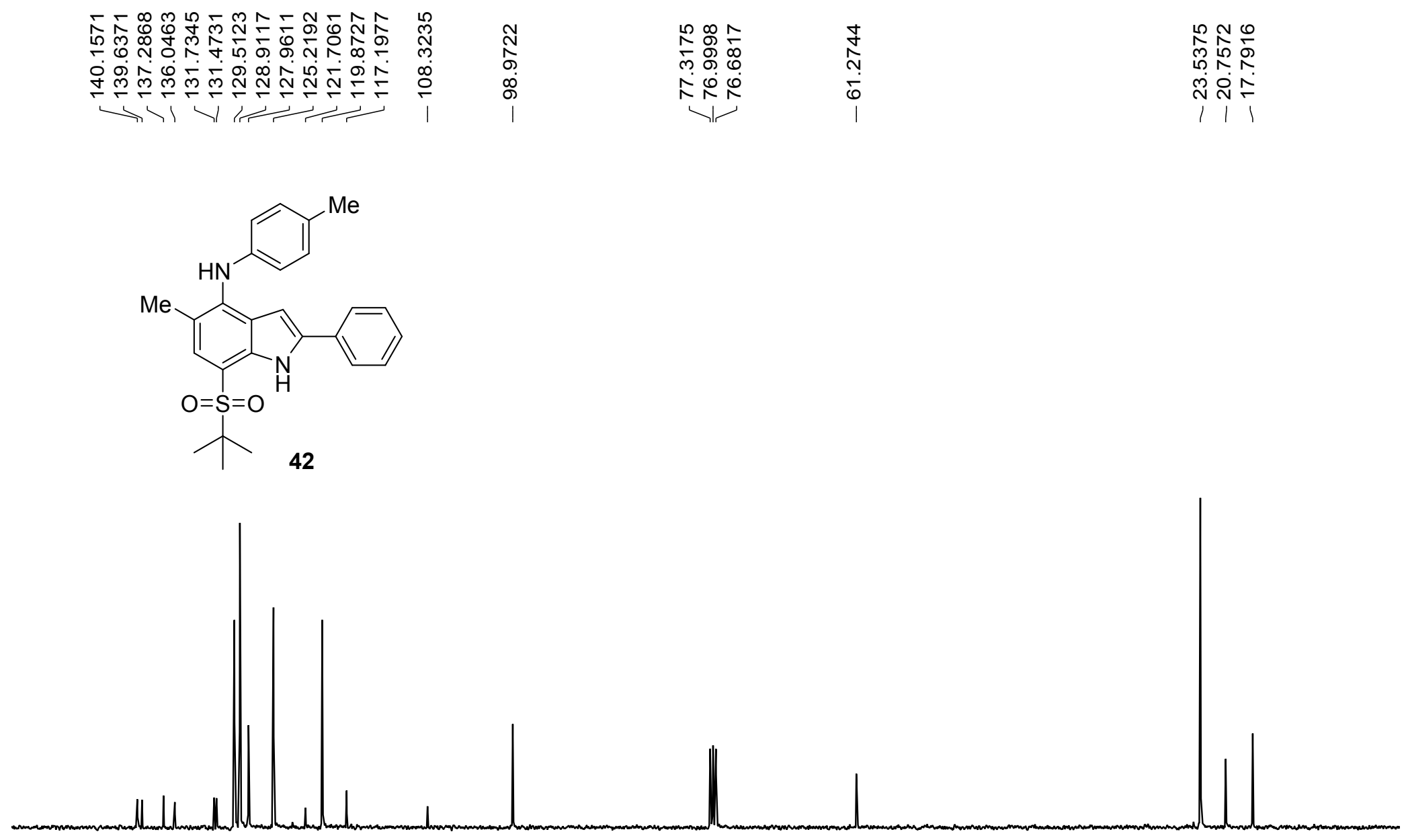